\title{
College and University Library
}

\section{Statistics, 1955-56}

$\mathrm{M}$ ORE LIBRARIES RETURNED the statistical questionnaire this year than ever before. Excluding the junior colleges, the questionnaires were mailed to 566 libraries in August. Only 246, or 43 per cent, returned them by the Committee's deadline of October 1, 1956. At that time, follow-up requests were sent to the 135 libraries whose data were included in previous compilations. By October 15, 1956, 177 additional reports had been received. Of the total received by this date, the Committee was able to use reports from 409 libraries. Approximately 100 institutions failed to return the statistical questionnaire.

The published data are essentially the same as last year. However, in an effort to interpret more clearly the figure representing per student operating expenditures, the Committee asked that extension students be reported separately. Even though librarians were requested to report the same figures as submitted to the U. S. Office of Education, there are still too many variables which distort these ratios. Also, the heading "total institutional expenditures" was changed to read "total educational and general institutional expenditures."

The libraries were grouped as in former years. Group I includes state universities as well as those institutions with fairly strong graduate programs. Group III includes libraries whose operating budgets are less than $\$ 35,000$.

The statistics were compiled again by members of the Committee, which included three new librarians whose names appear below. The Association owes these librarians a debt of gratitude for the great amount of time spent in producing these tables.

Mr. Bentz, chairman of the ACRL Statistics Committee, is associate director of the State University of Iowa Library.

\section{Group I: Compiled by James Ranz}

DIRECTOR OF LIBRARIES, UNIVERSITY OF WYOMING

Of the 126 questionnaires mailed to Group I libraries this year, 110 were returned, in varying degrees of completeness. All have been included in the prepared tables.

During the past year, library expenditures advanced markedly, but still lagged behind the increases in total institutional expenditures. Eighty-one institutions reported their total institutional expenditures and total library expenditures for both $1954 / 55$ and $1955 / 56$. Collectively, these 81 institutions spent 10 per cent more for their total operations in 1955/56 than they did in 1954/55, but only about $71 / 2$ per cent more for libraries.

Some interesting comparisons are also to be found in the salaries paid professional librarians in 1954/55 and in 1955/56. The beginning professional salary, if we take it to be identical with the median minimum salary paid "All Other Professional Assistants," increased from $\$ 3,336$ to $\$ 3,586$. The average salary for all professional librarians was \$4,422 in $1954 / 55, \$ 4,741$ in $1955 / 56$. (Figures result from multiplying the average of the median minimum and median maximum salaries for each category by the number of librarians within that category, adding the products thus secured for all categories, and dividing this sum by the total number of librarians in all categories.)

\section{Group II: Compiled by}

\section{J. Richard Blanchard}

\section{LibraRIAN, UNIVERSITY OF CALIFORNIA, Davis, California}

Questionnaires were returned by 97 libraries. Of these, four were received too late for tabulation. During the tabulation many mistakes were discovered and, it is hoped, corrected properly. A comparison with the Group 
II statistics for 1954/55 shows a continuing rise in enrollments, library collections, expenditures, and salaries. However, considering inflationary pressures and increasing enrollments, it would appear that budgets for the majority of Group II libraries are still far from adequate.

\section{Group III: Compiled by \\ Clarence Gorchels}

Associate Librarian,

Washington State College

Librarians represented in the Group III category again deserve congratulations for their cooperative attitude toward providing library statistics. This year 113 librarians (one more than last year) in this group responded with information. It is especially gratifying to note that the median salaries for professional librarians are well above the medians of last year. However, comparisons in this area, as in all others, must be made with considered judgment, since the libraries in this group show a good deal of variation in enrollment, type of library service given, amount of nonsalaried service, and interpretation of definitions in the statistical form.

\section{Teachers Colleges: Compiled by \\ Robert R. Hertel \\ Librarian, State University Teachers \\ College, Cortland, New York}

Ninety-six libraries returned questionnaires, and 94 libraries are included in the statistics. Since the deadline for inclusion was extended, some information which was reported in different form than that requested (e.g., total work hours for a professional librarian in the fiscal year) could not be corrected in time.

Fifty-nine libraries, comprising nearly 63 per cent of the total number reporting, include their extension enrollments in the computation of per student expenditures. Some teachers colleges do not have programs of extension courses which require library serv- ice or do not yet provide additional revenues to finance library service for existing programs. The increased service load of extension students has a significant effect upon the per student expenditure figure. Nearly 51 per cent of the libraries which support these extension programs reported a figure below the median of $\$ 29.07$. Administrators of teacher training libraries face many perplexing problems in financing and supplying extension services. It would be valuable for them to know the various bases for financing these services, whether by per capita, by per capita of full-time equivalents, by lump sum, or by some other means. A study of existing extension services is definitely needed for the guidance of teachers college librarians.

\section{Junior Colleges: Compiled by Ruth E. Scarborough \\ Librarian, Centenary Junior College, HacketTStown, New Jersey}

Statistics questionnaires were sent to all junior colleges in the country. Thirty-seven states and the District of Columbia are represented in the 147 colleges reporting, which is a 100 per cent increase over last year's returns. Unfortunately, 21 questionnaires were received after the deadline and could not be used.

Several items in the questionnaire differ from last year and from the other groups: student enrollment for day and evening, salary data in three divisions for the librarian, professional assistants and non-professional assistants, and number of months on duty. Since per student operating expenditure is figured on a total of day and evening students, this figure may seem small where the college has an unusually large evening enrollment. The enrollment was separated for day and evening students and footnoted for dormitory students to give a clearer picture of the college situation as it might affect the use of the library, size of library staff, etc.

A limited number of reprints of the $1955-56$ statistics are available from the ACRL office in Chicago at seventy-five cents a copy, or at fifty cents a copy if payment accompanies the order. 
Library $^{1}$

1. Alabama

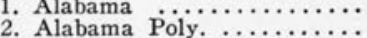

3. Arizona

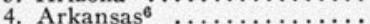

5. Baylor ...............

6. Boston College .........

7. Boston University $\ldots \ldots \ldots$.

9. Brooklyn College ........

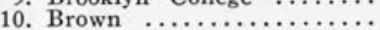

11. Buffalo California (Berkeley) ....

13. California (Davis) $\ldots \ldots \ldots$

14. California (Los Angeles) .

16. California (Santa Barbara)

17. California Inst. of Tech. .

19. Chicago $\ldots \ldots \ldots \ldots \ldots \ldots \ldots$

20. Cincinnati $\ldots \ldots \ldots \ldots \ldots \ldots$

21. City College (N.Y.) .....

23. Colorado ${ }^{10} \ldots \ldots \ldots \ldots \ldots \ldots \ldots$

25. Colorado A. \& M. . . . . . . .

26. Cornell

27. Dartmouth $\ldots \ldots \ldots \ldots \ldots \ldots$

28. Delaware $\ldots \ldots \ldots \ldots \ldots \ldots \ldots \ldots \ldots \ldots$

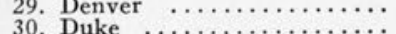

31. Florida . Florida State $\ldots \ldots \ldots \ldots \ldots \ldots \ldots \ldots \ldots$

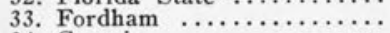

34. Georgia $\ldots \ldots \cdots \cdots$

36. Harvard $\ldots \ldots \ldots \ldots \ldots \ldots$

37. Hawaii ${ }^{13} \quad \ldots \ldots \ldots \ldots \ldots \ldots$

38. Houston $\ldots \ldots \ldots \ldots \ldots \ldots \ldots$

40. Illinois $\quad \ldots \ldots \ldots \ldots \ldots \ldots \ldots \ldots$

41. Indiana

42. Iowa

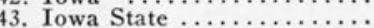

44. Johns Hopkins ..........

45. Joint University ........

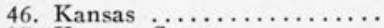

47. Kansas State . Ken . . . . . . . .

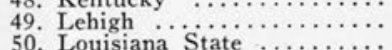

51. Maine

52. Marylandii $\ldots \ldots \ldots \ldots \ldots$

53. Massachusetts ..........

54. Mass. Inst. of Tech. . . . .

56. Michigan

57. Michigan state $\ldots \ldots \ldots \ldots \ldots$

58. Minnesota

59. Mississippi ${ }^{17} \ldots \ldots \ldots \ldots \ldots \ldots$

60. Mississippi State ..........

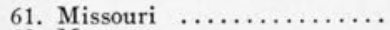

62. Montana . $3 . \ldots \ldots \ldots \ldots \ldots$

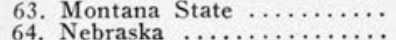

65. Nevada $\ldots \ldots \ldots \ldots \ldots \ldots \ldots \ldots$

66. New Hampshire $\ldots \ldots \ldots \ldots$

67. New Mexico ............

68. New York University ....

70. North Carolina State .....

71. North Dakota .........

72. Northwestern

73. Notre Dame ...........

74. Ohio State $\ldots \ldots \ldots \ldots \ldots$
Fiscal

Year
Ending

$30 \mathrm{Se} 56$

$30 \mathrm{Je} 56$

$30 \mathrm{Je} 56$

$30 \mathrm{Je} 56$

$30 \mathrm{Je} 56$

30 Je 56

$31 \mathrm{Mr} 56$

$30 \mathrm{Je} 56$

$30 \mathrm{Je} 56$

$30 \mathrm{Je} 56$

$30 \mathrm{Je} 56$

30 Je 56

$30 \mathrm{Je} 56$

$30 \mathrm{Je} 56$

30 Je 56

$30 \mathrm{Je} 56$

$31 \operatorname{Ag} 56$

$30 \mathrm{Je} 56$

$30 \mathrm{Je} 56$

30 Je 56

$30 \mathrm{Je} 56$

$30 \mathrm{Je} 56$

$30 \mathrm{Je} 56$

$30 \mathrm{Je} 56$

0Je56

$30 \mathrm{Je} 56$

$30 \mathrm{Je} 56$

$30 \mathrm{Je} 56$

30 Je 56

$30 \mathrm{Je} 56$

$30 \mathrm{Je} 56$

$30 \mathrm{Je} 56$

$31 \mathrm{Ag} 56$

$30 \mathrm{Je} 56$

30 Je56

$30 \mathrm{Je} 56$

$30 \mathrm{Je} 56$

30 Je56

30 A 56

$30 \mathrm{Je} 56$

$30 \mathrm{Je} 56$

$30 \mathrm{Je} 56$

$30 \mathrm{Je} 56$

$30 \mathrm{Je} 56$

$30 \mathrm{Je} 56$

$30 \mathrm{Je} 56$

$30 \mathrm{Je} 56$

$31 \mathrm{My} 56$

$30 \mathrm{Je} 56$

$30 \mathrm{Je} 56$

$30 \mathrm{Je} 56$

$30 \mathrm{Je} 56$
$30 \mathrm{Je} 56$

$30 \mathrm{Je} 56$

30 e 56

$30 \mathrm{Je} 56$

$30 \mathrm{Je} 56$

$30 \mathrm{Je} 56$

$30 \mathrm{Je} 56$

$30 \mathrm{Je} 56$

30 e 56

$30 \mathrm{Je} 56$

$30 \mathrm{Je} 56$

31 Ag 56

$30 \mathrm{Je} 56$

$30 \mathrm{Je} 56$
$30 \mathrm{Je} 56$
STUdent ENROLLMENT2

Trot

$\begin{array}{cccc}\text { Under- } & \text { Total } & \text { Total } & \text { Book } \\ \text { graduate } & \text { Graduate } & \text { Extension } & \text { Stock }\end{array}$

$\begin{array}{rrcl}6,993 & 569 & 4,034 & 534,007 \\ 7,372 & 541 & \ldots \dot{7}^{7} & 223,931 \\ 6,800 & 449 & 185 & 271,005 \\ 4,254 & 325 & 205 & 343,342 \\ 5,235 & 364 & \ldots . .^{7} & 218,293 \\ & & \ldots \ldots & 430,602^{8} \\ 6,401 & 694 & \ldots 364 & 508,847 \\ 9,232 & 716 & 1,764 & 324,946 \\ 6,080 & 323 & 1,048 & 270,788 \\ 16,725 & 1,622 & \ldots \ldots & 857,660 \\ 3,185 & 396 & \ldots \ldots & \end{array}$

Volumes
Added

News- Peri-

1,587

$98 \quad 3,415$

10,705

11,584

9,532

13,009

25,869

20,946

23,858

9,898

13,022

1,531

11,116

844

$259 \quad 332,253$

4,177

$15,815^{9}-2,142,801$

$7^{7} 131,315$

$\begin{array}{rr}1,165 & 1,159,728 \\ 80,238\end{array}$

6,797

13,947
64,998

14,745

1,945

569
2,001

1,645

13,233

76

$\ldots{ }^{7} \quad 87,171^{11}$

7,849

5,154
17,015

$\ldots{ }^{\top} \quad 516,434$

$2,339 \quad 1,925,754$

43,038

3,445

22,749

663

8,267

4,400
10,586

3,177
191
784

191
784
105

105
5,949

$5,800 \quad 446,774^{11}$

$\cdots, \quad 194,426$

$\cdots{ }^{7} \quad 835,681$

$\begin{array}{ll}\ldots{ }^{7} & 178,684 \\ \ldots & 2,164,652^{11}\end{array}$

8,315

2,790

2,133

4,819

3,481

1,405

218

1,152

$598 \quad 1,812,826$

i. $817 \quad 753,349$

720,093

.... $1,243,691$

$9,751 \quad 1,117$

$6,688^{34}$

7,054

5,021
4,863

1,117
819

819
2,434

460
294

4,452

4,812

10,532

11,759

21,015

11,240

7,642

8,106

3,701

7,065

5,581

6,035

2,701
8,569

3,397

13,296

4,004

3,657

12,850

15,239

20,163

2,611
3,718

8,084

2,646

2,651

7,186
1,805

3,108

4,239

18,038

5,036
4,417

$1,675 \quad 700,274$

$1,183 \quad 447,929$

$1,229 \quad 325,335$

$\ddot{1,47 \dot{7}^{7}} \begin{array}{ll}339,993 \\ \end{array}$

5,954

368

1,104

1,316

3,114

2,379

1,689
972

1,118

2,237

987

429

1,005
533

533
1,388

100

2,448
337

1,991

657

... 6,085,761

$1,161 \quad 264,747$

$1,694 \quad 158,663$

$\ddot{2,635} \quad 2,978,597$

$6,2551,059,850$

$\begin{array}{ll}77 & 898,041\end{array}$

$\cdots \cdots, 1,076,266$

$182 \quad 691,882$

$257 \quad 204,499$

$\begin{array}{ll}\ldots{ }^{7} & 712,612 \\ & 354,220^{8}\end{array}$

$\begin{array}{ll}55 \mathrm{i} & \mathbf{6 9 0 , 5 2 9}\end{array}$

$1,023 \quad 276,126$

$3,710^{35} \quad 317,563$

… 199,830

… $\quad 588,835$

$3,369 \quad 2,411,628$

$7,801^{15}$

1,778

3,230
223

214

899

210

148
627

46

171
789

789

12,911
1,539

3,024

$16,181^{43}$

5,051

19,048
9,816

323

1,281
633

2,696
1,063

19,460

16,722

2,440

34,596
4,304 
Statistics, I $955^{-1} 95^{6}$ (Group I)

\begin{tabular}{|c|c|c|c|c|c|c|c|c|c|c|}
\hline $\begin{array}{c}\text { Staff } \\
\text { Salaries }\end{array}$ & $\begin{array}{l}\text { Student } \\
\text { Service }\end{array}$ & $\begin{array}{l}\text { Total } \\
\text { Staff } \\
\text { Salaries } \\
\text { and } \\
\text { Student } \\
\text { Service N }\end{array}$ & $\begin{array}{l}\text { Books } \\
\text { and } \\
\text { Related } \\
\text { Materials }\end{array}$ & Binding & $\begin{array}{l}\text { Total for } \\
\text { Books } \\
\text { and } \\
\text { Binding }\end{array}$ & $\begin{array}{l}\text { Other } \\
\text { Operat- } \\
\text { ing Ex- } \\
\text { pendi- } \\
\text { tures }^{3}\end{array}$ & $\begin{array}{c}\text { Total } \\
\text { Operating } \\
\begin{array}{c}\text { Expendi- } \\
\text { tures }\end{array}\end{array}$ & $\begin{array}{l}\text { Per Stu- } \\
\text { dent } \\
\text { Operat- } \\
\text { ing Ex- } \\
\text { pendi- } \\
\text { tures }^{41}\end{array}$ & $\begin{array}{l}\text { Total Edu- } \\
\text { cational and } \\
\text { General In- } \\
\text { stitutional } \\
\text { Expendi- } \\
\text { tures }\end{array}$ & $\begin{array}{l}\text { Ratio of Li- } \\
\text { brary Ex- } \\
\text { penditures } \\
\text { to Total } \\
\text { (Per Cent) }\end{array}$ \\
\hline $\begin{array}{r}186,249 \$ \\
85,410 \\
84,491 \\
85,389 \\
72,080\end{array}$ & $\begin{array}{r}27,121 \$ 9 \\
9,074 \\
21,764 \\
12,775 \\
29,725\end{array}$ & $\begin{array}{r}213,370 \\
94,484 \\
106,255 \\
98,164 \\
101,805\end{array}$ & $\begin{array}{r}\$ 123,982 \$ \\
46,950 \\
53,733 \\
47,937 \\
48,825\end{array}$ & $\begin{array}{r}18,350 \\
7,490 \\
13,258 \\
5,668 \\
10,045\end{array}$ & $\begin{array}{r}\$ 142,332 \$ \\
54,440 \\
66,991 \\
53,605 \\
58,870\end{array}$ & $\begin{array}{r}15,559 \\
7,343 \\
10,560 \\
7,160 \\
15,665\end{array}$ & $\begin{array}{l}371,261 \\
156,267 \\
183,806 \\
158,929 \\
176,340\end{array}$ & $\begin{array}{l}\$ 49.10 \\
19.75 \\
25.36 \\
34.71 \\
31.49\end{array}$ & $\begin{array}{r}\$ 7,361,237 \\
8,113,538 \\
5,401,123 \\
4,473,782 \\
2,656,195\end{array}$ & $\begin{array}{l}5.0 \\
1.9 \\
3.4 \\
3.6 \\
6.6\end{array}$ \\
\hline $\begin{array}{r}99,231 \\
189,583 \\
183,623 \\
182,800 \\
196,402\end{array}$ & $\begin{array}{r}12,093 \\
31,838 \\
7,628 \\
57,885 \\
19,397\end{array}$ & $\begin{array}{l}128,824^{23} \\
221,421 \\
191,251 \\
240,685 \\
215,799\end{array}$ & $\begin{array}{r}52,407 \\
77,663 \\
112,384 \\
56,152 \\
91,696\end{array}$ & $\begin{array}{r}3,536 \\
10,334 \\
21,898 \\
5,544 \\
14,083\end{array}$ & $\begin{array}{r}55,943 \\
87,997 \\
134,282 \\
61,696 \\
105,779\end{array}$ & $\begin{array}{l}12,433 \\
11,314 \\
12,830 \\
11,459 \\
20,912\end{array}$ & $\begin{array}{l}197,200 \\
320,732 \\
338,363 \\
313,840 \\
342,490\end{array}$ & $\begin{array}{l}27.79 \\
32.24 \\
52.84 \\
17.11 \\
95.64\end{array}$ & $\begin{array}{l}3,014,551 \\
\dot{6} \ddot{4} \ddot{7} 0 \dot{8}^{7} \\
8,612,281 \\
5,339,116\end{array}$ & $\begin{array}{l}6.5 \\
5.2 \\
3.6 \\
6.4\end{array}$ \\
\hline $\begin{array}{c}105,736 \\
1,310,312 \\
114,175 \\
720,67516 \\
81,146\end{array}$ & $\begin{array}{c}7,834 \\
254,827 \\
27,272 \\
199,64916 \\
17,416\end{array}$ & $\begin{array}{c}113,570 \\
1,565,139 \\
141,447 \\
920,324^{16} \\
98,562\end{array}$ & $\begin{array}{r}32,388 \\
370,524 \\
77,001 \\
301,425 \\
73,794\end{array}$ & $\begin{array}{r}5,500 \\
93,533 \\
24,500 \\
81,195 \\
10,718\end{array}$ & $\begin{array}{r}37,888 \\
464,057 \\
101,501 \\
382,620 \\
84,512\end{array}$ & $\begin{array}{c}7,150 \\
120,029 \\
13,740 \\
94,008^{16} \\
12,734\end{array}$ & $\begin{array}{r}158,608 \\
2,149,225 \\
256,688 \\
1,396,952 \\
195,808\end{array}$ & $\begin{array}{r}14.77 \\
124.96 \\
131.97 \\
97.85 \\
275.00\end{array}$ & $\begin{array}{c}6,543,873 \\
\dot{8}, 13 \dot{13}, 4 \dot{4}^{7} \\
20,851,101^{16} \\
3,600,000^{16}\end{array}$ & $\begin{array}{l}2.4 \\
3.2 \\
6.7 \\
5.4\end{array}$ \\
\hline $\begin{array}{r}88,053 \\
62,548 \\
129,567 \\
419,934 \\
158,583\end{array}$ & 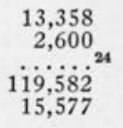 & $\begin{array}{c}101,411 \\
65,148 \\
131,067^{25} \\
539,516 \\
174,160\end{array}$ & $\begin{array}{r}31,327 \\
33,080 \\
45,498 \\
152,809 \\
84,023\end{array}$ & $\begin{array}{l}7,063 \\
8,718 \\
1,320^{26} \\
40,058 \\
10,623\end{array}$ & $\begin{array}{r}38,390 \\
41,798 \\
46,818 \\
192,867 \\
94,646\end{array}$ & $\begin{array}{r}6,585 \\
9,619 \\
10,257 \\
44,208 \\
6,375\end{array}$ & $\begin{array}{l}146,386 \\
116,565 \\
188,143 \\
776,591 \\
275,181\end{array}$ & $\begin{array}{r}72.43 \\
120.42 \\
53.07 \\
152.57 \\
19.56\end{array}$ & $\begin{array}{c}\dot{8}, 347,547^{7} \\
3,454,674 \\
28,259,632^{39} \\
8,531,000\end{array}$ & $\begin{array}{l}1.4 \\
5.4 \\
2.7 \\
3.2\end{array}$ \\
\hline $\begin{array}{r}316,133 \\
23,340 \\
154,821 \\
62,131 \\
903,351\end{array}$ & $\begin{array}{c}37,360 \\
4,491 \\
37,591 \\
10,927 \\
\ldots \ldots .24\end{array}$ & $\begin{array}{r}353,493 \\
27,831 \\
192,412 \\
73,058 \\
903,351\end{array}$ & $\begin{array}{r}70,850 \\
16,885 \\
95,681 \\
19,273 \\
263,983\end{array}$ & $\begin{array}{r}12,553 \\
1,897 \\
12,090 \\
5,517 \\
65,500\end{array}$ & $\begin{array}{r}83,403 \\
18,782 \\
107,771 \\
24,790 \\
329,483\end{array}$ & $\begin{array}{r}12,593 \\
2,254 \\
7,701 \\
5,507 \\
60,961\end{array}$ & $\begin{array}{r}449,489 \\
48,867 \\
307,884 \\
103,355 \\
1,293,795\end{array}$ & $\begin{array}{l}17.34 \\
57.22 \\
34.02 \\
22.94 \\
48.76\end{array}$ & $\begin{array}{r}11,419,906 \\
800,993 \\
8,005,888 \\
\ldots \ldots \ldots .7 \\
\ldots \ldots \ldots 7^{7}\end{array}$ & $\begin{array}{l}3.9 \\
6.1 \\
3.8 \\
\ldots \\
\cdots\end{array}$ \\
\hline $\begin{array}{r}600,947 \\
198,518 \\
66,255 \\
100,312 \\
260,965\end{array}$ & $\begin{array}{r}108,546 \\
10,924 \\
4,295 \\
11,918 \\
30,426\end{array}$ & $\begin{array}{r}709,493 \\
209,442 \\
70,550 \\
112,230 \\
291,391\end{array}$ & $\begin{array}{r}246,650 \\
79,143 \\
56,784 \\
41,063 \\
\ldots \ldots .7\end{array}$ & $\begin{array}{r}48,870 \\
17,398 \\
2,379 \\
5,493 \\
\ldots \ldots \ldots\end{array}$ & $\begin{array}{r}295,520 \\
96,541 \\
59,163 \\
46,556 \\
224,963\end{array}$ & $\begin{array}{c}54,255 \\
41,983^{27} \\
5,754 \\
4,431 \\
41,561\end{array}$ & $\begin{array}{c}1,059,268 \\
347,966^{27} \\
135,467 \\
163,217 \\
557,915\end{array}$ & $\begin{array}{r}108.98 \\
115.68 \\
44.58 \\
27.33 \\
107.71\end{array}$ & $\begin{array}{r}32,167,533 \\
5,362,390 \\
4,465,020 \\
3,419,949 \\
10,634,239\end{array}$ & $\begin{array}{l}3.3 \\
6.5 \\
3.0 \\
4.8 \\
5.3\end{array}$ \\
\hline $\begin{array}{l}344,834 \\
219,587 \\
130,470 \\
165,548 \\
132,490\end{array}$ & $\begin{array}{l}57,199 \\
12,679 \\
18,000 \\
10,915 \\
11,437\end{array}$ & $\begin{array}{l}402,033 \\
232,266 \\
148,470 \\
176,463 \\
143,927\end{array}$ & $\begin{array}{r}221,204 \\
98,484 \\
56,460 \\
85,221 \\
58,636\end{array}$ & $\begin{array}{r}26,056 \\
10,873 \\
7,360 \\
12,375 \\
11,465\end{array}$ & $\begin{array}{r}247,260 \\
109,357 \\
63,820 \\
97,596 \\
70,101\end{array}$ & $\begin{array}{r}32,357 \\
15,777 \\
9,841 \\
10,444 \\
25,557\end{array}$ & & $\begin{array}{l}62.72 \\
47.61 \\
23.41 \\
51.91 \\
46.46\end{array}$ & $\begin{array}{r}16,783,552 \\
6,270,935 \\
4,960,000 \\
4,281,344 \\
6,419,521\end{array}$ & $\begin{array}{l}4.1 \\
5.7 \\
4.5 \\
6.6 \\
3.7\end{array}$ \\
\hline $\begin{array}{r}1,247,016 \\
107,040 \\
140,167 \\
129,131 \\
952,480\end{array}$ & 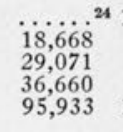 & $\begin{array}{r}1,247,016 \\
125,708 \\
169,238 \\
165,791 \\
1,048,413\end{array}$ & $\begin{array}{r}479,973 \\
42,237 \\
63,523 \\
24,156 \\
\ldots \ldots \ldots\end{array}$ & $\begin{array}{r}115,401 \\
12,156 \\
8,596 \\
2,574 \\
\ldots \ldots \ldots\end{array}$ & $\begin{array}{r}595,374 \\
54,393 \\
72,119 \\
26,730 \\
491,554\end{array}$ & $\begin{array}{r}332,219 \\
7,928 \\
18,379 \\
1,459 \\
52,987\end{array}$ & $\begin{array}{r}2,174,609 \\
188,029 \\
259,736 \\
193,980 \\
1,592,954\end{array}$ & $\begin{array}{r}208.98 \\
36.30 \\
22.32 \\
14.84 \\
66.02\end{array}$ & $\begin{array}{c}39,331,081 \\
5,264,663 \\
5,103,745 \\
5,800,000^{16} \\
54,238,782\end{array}$ & $\begin{array}{l}5.5 \\
3.6 \\
5.1 \\
3.3 \\
2.9\end{array}$ \\
\hline $\begin{array}{l}365,000^{16} \\
270,910 \\
154,685 \\
210,775 \\
128,851\end{array}$ & $\begin{array}{l}51,000^{16} \\
46,025 \\
24,157 \\
20,696 \\
37,690\end{array}$ & $\begin{array}{l}416,000^{16} \\
316,935 \\
178,842 \\
231,771^{28} \\
166,541\end{array}$ & $\begin{array}{c}250,000^{16} \\
172,200 \\
74,933 \\
76,849 \\
79,660\end{array}$ & $\begin{array}{l}43,000^{16} \\
24,157 \\
24,993 \\
17,600 \\
16,376\end{array}$ & $\begin{array}{c}293,000^{16} \\
196,357 \\
99,926 \\
94,449 \\
96,036\end{array}$ & $\begin{array}{c}24,000^{16} \\
29,717 \\
9,239 \\
17,400 \\
11,221\end{array}$ & $\begin{array}{l}733,000^{16} \\
543,009 \\
288,007 \\
343,620 \\
273,798\end{array}$ & $\begin{array}{l}53.82 \\
58.19 \\
31.73 \\
48.98 \\
46.11\end{array}$ & $\begin{array}{r}11,899,533 \\
13,568,787 \\
14,218,496 \\
5,824,514\end{array}$ & $\begin{array}{l}4.6 \\
2.1 \\
2.4 \\
4.7\end{array}$ \\
\hline $\begin{array}{r}246,506 \\
96,770 \\
170,662 \\
52,564 \\
309,593\end{array}$ & $\begin{array}{r}63,590 \\
18,000 \\
15,987 \\
7,455 \\
38,269\end{array}$ & $\begin{array}{r}310,096 \\
114,770 \\
186,649 \\
60,019 \\
347,862\end{array}$ & $\begin{array}{r}213,013 \\
57,886 \\
99,581 \\
36,890 \\
180,949\end{array}$ & $\begin{array}{r}19,202 \\
6,114 \\
17,093 \\
9,308 \\
27,052\end{array}$ & $\begin{array}{r}232,215 \\
64,000 \\
116,674 \\
46,198 \\
208,001\end{array}$ & $\begin{array}{r}42,808 \\
5,421 \\
19,835 \\
7,311 \\
28,536\end{array}$ & $\begin{array}{l}585,119 \\
184,191 \\
323,158 \\
113,528 \\
584,399\end{array}$ & $\begin{array}{l}72.67 \\
30,65 \\
45.90 \\
35.10 \\
58.69\end{array}$ & $\begin{array}{r}10,155,960 \\
8,863,291 \\
3 \ddot{3} \ddot{4}, 9 \dot{5}^{7} \\
18,458,240\end{array}$ & $\begin{array}{l}5.8 \\
2.1 \\
3.0 \\
3.2\end{array}$ \\
\hline $\begin{array}{r}35,148 \\
171,412 \\
49,783 \\
177,201 \\
211,151\end{array}$ & $\begin{array}{r}11,120 \\
29,115 \\
5,483 \\
19,075 \\
17,532\end{array}$ & $\begin{array}{r}46,268 \\
200,527 \\
55,266 \\
196,276 \\
228,683\end{array}$ & $\begin{array}{r}28,965 \\
128,470 \\
41,513 \\
61,826 \\
158,217\end{array}$ & $\begin{array}{r}3,525 \\
14,723 \\
9,205 \\
13,000 \\
16,660\end{array}$ & $\begin{array}{r}32,490 \\
143,193 \\
50,718 \\
74,826 \\
174,877\end{array}$ & $\begin{array}{r}1,427 \\
12,851 \\
5,263 \\
10,764 \\
13,890\end{array}$ & & $\begin{array}{l}22.93 \\
22.65 \\
25.63 \\
49.91 \\
34.37\end{array}$ & $\begin{array}{c}4,557,955 \\
21,117,963^{42} \\
7,216,091 \\
24,591,475^{36} \\
7,519,060\end{array}$ & $\begin{array}{l}1.8 \\
1.7 \\
1.5 \\
1.1 \\
5.6\end{array}$ \\
\hline $\begin{array}{r}803,457 \\
196,077 \\
513,055 \\
60,058 \\
54,209\end{array}$ & $\begin{array}{r}119,020 \\
79,387 \\
109,304 \\
9,770 \\
8,196\end{array}$ & $\begin{array}{r}275,464 \\
622,359 \\
69,828 \\
62,405\end{array}$ & $\begin{array}{r}307,261 \\
164,000 \\
263,543 \\
22,116 \\
18,057\end{array}$ & $\begin{array}{r}47,573 \\
18,117 \\
50,539 \\
6,663 \\
8,620\end{array}$ & $\begin{array}{r}354,834 \\
182,117 \\
314,082 \\
28,779 \\
26,677\end{array}$ & $\begin{array}{r}57,482 \\
3,100 \\
4,527\end{array}$ & $\begin{array}{r}1,369,078 \\
477,548 \\
993,923 \\
101,707 \\
93,609\end{array}$ & & $\begin{array}{r}31,92 \\
21,05 \\
38,79 \\
2,35 \\
7,72\end{array}$ & $\begin{array}{l}4.3 \\
2.3 \\
2.6 \\
4.3 \\
1.2\end{array}$ \\
\hline $\begin{array}{r}147,293 \\
55,108 \\
51,850 \\
245,062 \\
42,280\end{array}$ & $\begin{array}{r}21,997 \\
7,065 \\
7,000 \\
36,650 \\
5,020\end{array}$ & $\begin{array}{r}169,290 \\
62,173 \\
58,850 \\
281,712 \\
47,300\end{array}$ & $\begin{array}{r}156,905 \\
18,143 \\
23,000 \\
112,660 \\
16,080\end{array}$ & $\begin{array}{r}20,314 \\
4,350 \\
4,000 \\
16,397 \\
2,020\end{array}$ & $\begin{array}{r}177,219 \\
22,493 \\
27,000 \\
129,057 \\
18,100\end{array}$ & $\begin{array}{r}22,653 \\
2,903 \\
2,660 \\
19,669 \\
2,164\end{array}$ & $\begin{array}{r}369,162 \\
87,569 \\
88,510 \\
430,438 \\
67,564\end{array}$ & $\begin{array}{l}10 \\
66 \\
62 \\
09 \\
50\end{array}$ & $\begin{array}{r}2,255,037^{7} \\
13,919,38 \dot{9}^{7} \\
2,102,853\end{array}$ & $\begin{array}{l}3.9 \\
3.1 \\
3.2\end{array}$ \\
\hline $\begin{array}{r}64,232 \\
89,993 \\
465,062 \\
297,199 \\
86,806\end{array}$ & $\begin{array}{l}13,117 \\
18,412 \\
3 \ddot{3}, 69 \dot{4}^{7} \\
11,885\end{array}$ & $\begin{array}{r}77,349 \\
108,405 \\
465,062 \\
330,893 \\
98,691\end{array}$ & $\begin{array}{r}28,930 \\
45,972 \\
114,154 \\
122,415 \\
69,854\end{array}$ & $\begin{array}{r}5,392 \\
9,383 \\
18,836 \\
17,519 \\
9,699\end{array}$ & $\begin{array}{r}34,322 \\
55,355 \\
132,990 \\
139,934 \\
79,553\end{array}$ & $\begin{array}{r}6,328 \\
8,262 \\
50,620 \\
33,186 \\
4,607\end{array}$ & & $\begin{array}{l}35.99 \\
34.21 \\
20.96 \\
76.66 \\
37.99\end{array}$ & $\begin{array}{r}4,289 \\
3,642 \\
32,951 \\
11,705 \\
12,995\end{array}$ & $\begin{array}{l}2.8 \\
4.7 \\
2.0 \\
4.3 \\
1.4\end{array}$ \\
\hline $\begin{array}{r}39,533 \\
332,477 \\
146,862 \\
474,434 \\
182,174\end{array}$ & $\begin{array}{r}8,723 \\
53,297 \\
21,083 \\
62,130 \\
26,513\end{array}$ & $\begin{array}{r}48,256 \\
385,774 \\
167,945 \\
536,564 \\
208,687\end{array}$ & $\begin{array}{c}40,732 \\
102,800^{7} \\
174,292 \\
147,506\end{array}$ & $\begin{array}{c}6,497 \\
\dddot{11}, 51 \dot{8}^{7} \\
9,556^{26} \\
18,822\end{array}$ & $\begin{array}{r}47,229 \\
230,557 \\
114,318 \\
183,848 \\
166,328\end{array}$ & $\begin{array}{r}6,772 \\
42,567 \\
9,936 \\
34,623 \\
22,745\end{array}$ & $\begin{array}{l}102,257 \\
658,898 \\
292,199 \\
755,035 \\
397,760\end{array}$ & $\begin{array}{l}30.55 \\
61.36^{37} \\
51.41 \\
34.72 \\
36.56\end{array}$ & $\begin{array}{c}2,047,000 \\
15,809,339 \\
9,936,000 \\
33,806,787 \\
6,320,305^{29}\end{array}$ & $\begin{array}{l}5.0 \\
4.2 \\
2.9 \\
2.2 \\
5.8\end{array}$ \\
\hline
\end{tabular}




\section{Library ${ }^{1}$}

76. Oregon ${ }^{20}$

77. Oregon State

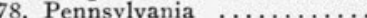

79. Pennsylvania State .......

80. Pittsburgh ...........

81. Princeton $\ldots \ldots \ldots \ldots \ldots \ldots$

82. Purdue

83. Rhode Island $\ldots \ldots \ldots \ldots \ldots$

84. Rice $\ldots \ldots \ldots \ldots \ldots \ldots \ldots$

85. Rochester ............

86. Rutgers $\ldots \ldots \ldots \ldots \ldots \ldots$

87. Saint Louis...$\ldots \ldots \ldots \ldots$

88. South Carolina ............

90. Southern California ........

91. Southern Illinois $\ldots \ldots \ldots$.

92. Southern Methodist ......

94. Temple $\ldots \ldots \ldots \ldots \ldots \ldots \ldots \ldots$

95. Tennessee $\ldots \ldots \ldots \ldots \ldots \ldots$

96. Texas

97. Texas A. \& $\dot{M} . \ldots \ldots \ldots \ldots$

98. Utah $\ldots \ldots \ldots \ldots \ldots \ldots \ldots$

99. Utah State $\ldots \ldots \ldots \ldots \ldots \ldots \ldots$

101. Virginia ${ }^{21} \quad \ldots \ldots \ldots \ldots \ldots$

102. Washington (St Louis) ...

104. Washington State ...........

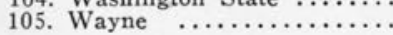

106. West Virginia ${ }^{17} \ldots \ldots \ldots$

107. Western Reserve .......

108. Wisconsin $\ldots \ldots \ldots \ldots \ldots \ldots$

110. Yale..$\ldots \ldots \ldots \ldots \ldots \ldots$

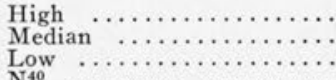

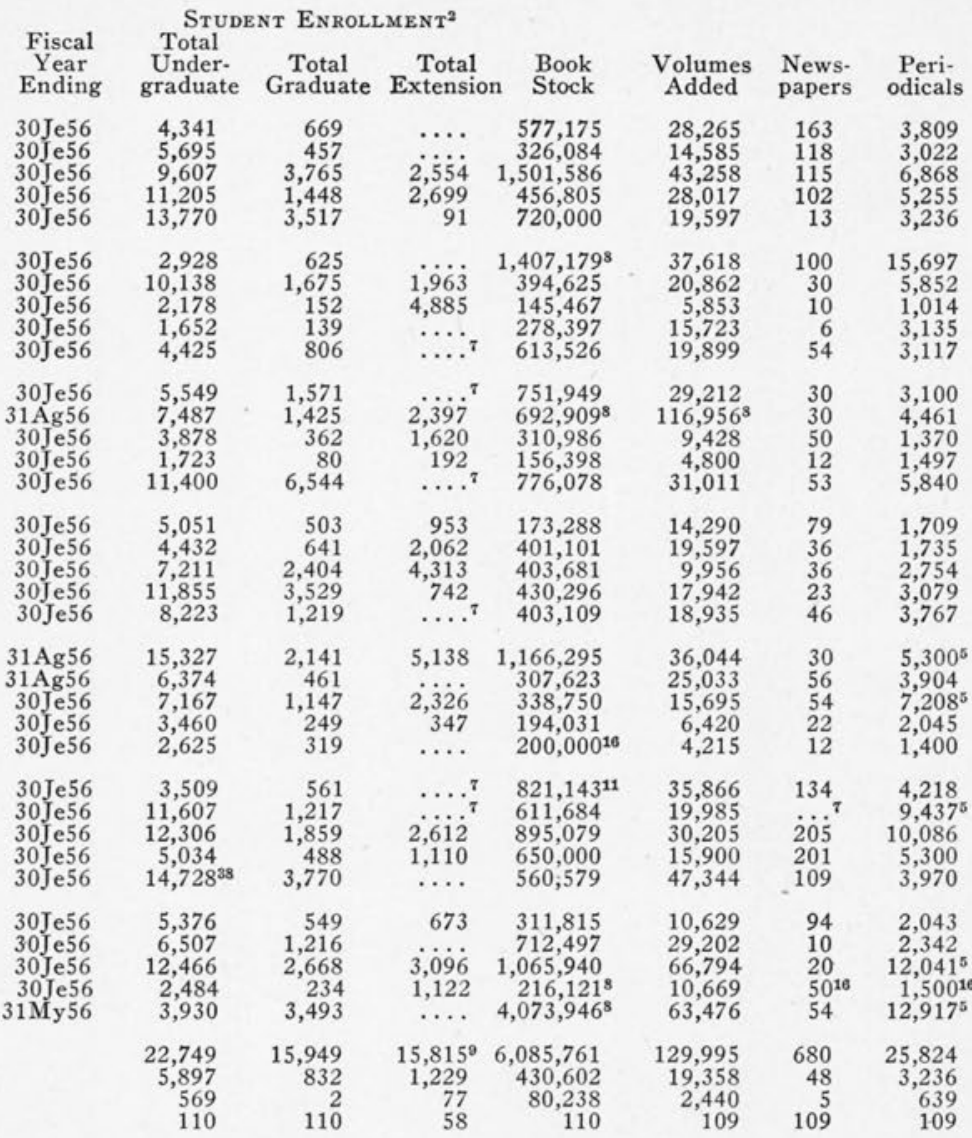

1. Central library and all agencies. 2. Resident and special students (no student counted twice). 3. Includes supplies, transportation and all other non-capital expenditures not specifically reported. 4. Includes expenditures for administration, resident instruction, organized research, extension, libraries, plant operation and mainteriance, etc., but excludes capital costs and expenditures for auxiliary enterprises such as dormitories, cafeterias, etc. 5. Includes serials. 6. Excludes Schools of Medicine, Pharmacy and Nursing. 7. Not reported or not available. 8. Adjusted figure. 9. Includes entire Northern Division, University of California. 10. Excludes Denison M. D. Memorial Library. 11. Excludes microfilm. 12. Excludes Barnard, Teachers College, New York School of Social Work, and College of Pharmacy, except for enrollment figures. 13. Excludes T. C. Elementary and High Schools, Hilo Branch, Legislative Reference Bureau, and Industrial Relations. 14. Excludes Maryland State College. Hilo Branch, Legislative Reference Bureau, and Industrial Relations. 14. Excludes Maryland State College.
15. Includes professional students. 16. Estimate. 17. Excludes Schools of Law and Medicine. 18. Includes Division of Health Affairs except for library expenditures. 19. Includes duplicates. 20. Excludes Schools of Medi-

\section{College and University Library Salary}

\begin{tabular}{|c|c|c|c|c|c|c|c|c|c|c|}
\hline \multirow[b]{2}{*}{ Library ${ }^{1}$} & \multirow[b]{2}{*}{$\underset{\text { brarian }}{\mathrm{Li}-}$} & \multicolumn{3}{|c|}{$\begin{array}{c}\text { Associate OR } \\
\text { AssISTANT } \\
\text { CHIEF LIBRARIAN }\end{array}$} & \multicolumn{2}{|c|}{ DEPARTMENT } & \multirow{2}{*}{$\begin{array}{c}\text { HEADS } \\
\text { Num- } \\
\text { ber }\end{array}$} & \multicolumn{3}{|c|}{$\begin{array}{c}\text { HEAD LRRARAN } \\
\text { SCHOLL, CoLLEGE, } \\
\text { AND DEPARTMENTIL } \\
\text { (PROFESSIONAL) }\end{array}$} \\
\hline & & $\begin{array}{l}\text { Mini- } \\
\text { mum }\end{array}$ & $\begin{array}{l}\text { Maxi- } \\
\text { mum }\end{array}$ & & $\begin{array}{l}\text { Mini- } \\
\text { mum }\end{array}$ & $\begin{array}{l}\text { Maxi- } \\
\text { mum }\end{array}$ & & $\begin{array}{l}\text { Mini- } \\
\text { mum }\end{array}$ & & \\
\hline $\begin{array}{l}\text { Poly } \\
a^{5} \ldots \\
\ldots \ldots\end{array}$ & $\begin{array}{l}\$ \ldots . .4 \\
7.000^{4} \\
7,600 \\
\ldots \ldots\end{array}$ & $\begin{array}{l}\$ \ldots \\
3,900 \\
6,250\end{array}$ & $\ldots$ & $\begin{array}{l}1 \\
.1 \\
1 \\
. .4\end{array}$ & $\begin{array}{c}\$ 4,000 \\
4,750^{4} \\
4,750 \\
\ldots . .4\end{array}$ & $\begin{array}{c}\$ 6,000 \\
\ldots,{ }^{4} \\
5,400 \\
4,800 \\
\ldots \ldots\end{array}$ & $\begin{array}{r}8 \\
4 \\
3 \\
3 \\
\cdots\end{array}$ & \begin{tabular}{c}
$\$ \ldots{ }^{11}$ \\
\hdashline, 900 \\
3,600
\end{tabular} & $\dddot{4,600}$ &.$^{11}$ \\
\hline $\begin{array}{l}\text { Boston Colle } \\
\text { Boston Univ Un } \\
\text { British Colu } \\
\text { Brooklyn Co } \\
\text { Brown ..... }\end{array}$ & $\begin{array}{r}7,500 \\
\ldots \ldots \\
11,300 \\
9,000\end{array}$ & $\begin{array}{l}5,550 \\
\cdots \cdots \\
6,450^{7}\end{array}$ & \begin{tabular}{l}
$\ldots \ldots$ \\
\hdashline, $950^{8}$ \\
8,96
\end{tabular} & $\begin{array}{l}1 \\
\dot{2} \\
1\end{array}$ & $\begin{array}{c}\ldots \ldots .4 \\
4,700 \\
4,100 \\
3,960\end{array}$ & $\begin{array}{l}\ldots \ldots . \\
\because, 000 \\
7,350 \\
6,000\end{array}$ & \begin{tabular}{l}
$\because \because 4$ \\
\hdashline 4 \\
8 \\
6
\end{tabular} & $\begin{array}{c}3,600 \\
\ldots \ldots \\
\ldots \ldots \\
3,480\end{array}$ & $\begin{array}{l}6,400 \\
\ldots \ldots \\
\ldots \ldots \\
4,080\end{array}$ & 7 \\
\hline $\begin{array}{l}\text { uffalo } \\
\text { alifornia (Berkeley) } \\
\text { alifornia } \\
\text { alifornia (Davis) } \\
\text { alifornia (River Angeles) } \\
\text { aliverside) }\end{array}$ & $\begin{array}{r}15,900^{1} \\
8,940 \\
14,400 \\
8,940\end{array}$ & $\begin{array}{l}9,344^{4} \\
7,008 \\
7,356 \\
4,512\end{array}$ & $\begin{aligned} 10,860 \\
\ddot{8}, 940 \\
6,060\end{aligned}$ & $\begin{array}{l}1 \\
3 \\
1 \\
2 \\
2\end{array}$ & $\begin{array}{l}4,795 \\
4,980 \\
4,512 \\
7,008 \\
4,092\end{array}$ & $\begin{array}{r}5,840 \\
11,700 \\
6,060 \\
9,384 \\
6,060\end{array}$ & $\begin{array}{r}3 \\
26 \\
4 \\
5 \\
3\end{array}$ & $\begin{array}{l}3,625 \\
4,312^{11} \\
4,296 \\
7,356\end{array}$ & $\begin{array}{l}4,000 \\
\because 6,11 \\
6,060 \\
8,940 \\
\cdots \cdots\end{array}$ & $\begin{array}{l}3 \\
2^{11} \\
11\end{array}$ \\
\hline
\end{tabular}

Head Librarian

School, College, AND DePART MENTAL (PROFESSIONAL) 


\begin{tabular}{|c|c|c|c|c|c|c|c|c|c|c|c|}
\hline$\underset{\text { Staffiaries }}{\text { Stal }}$ & $\begin{array}{l}\text { Student } \\
\text { Service }\end{array}$ & $\begin{array}{l}\text { Total } \\
\text { Staff } \\
\text { Salaries } \\
\text { and } \\
\text { Student } \\
\text { Service A }\end{array}$ & $\begin{array}{l}\text { Books } \\
\text { and } \\
\text { Related } \\
\text { Materials }\end{array}$ & Binding & $\begin{array}{l}\text { Total for } \\
\text { Books } \\
\text { and } \\
\text { Binding }\end{array}$ & $\begin{array}{l}\text { Other } \\
\text { Operat- } \\
\text { ing Ex- } \\
\text { pendi- } \\
\text { tures }^{3}\end{array}$ & $\begin{array}{c}\text { Total } \\
\text { Operating } \\
\text { Expendi- } \\
\text { tures }\end{array}$ & $\begin{array}{l}\text { Per Stu- } \\
\text { dent } \\
\text { Operat- } \\
\text { ing Ex- } \\
\text { pendi- } \\
\text { tures }^{41}\end{array}$ & $\begin{array}{l}\text { Total Edu- } \\
\text { cational and } \\
\text { General In- } \\
\text { stitutional } \\
\text { Expendi- } \\
\text { tures }\end{array}$ & $\begin{array}{l}\text { Ratio of Li- } \\
\text { brary Ex- } \\
\text { penditures } \\
\text { to Total } \\
\text { (Per Cent) }\end{array}$ & \\
\hline $\begin{array}{l}200,792 \\
115,162 \\
464,642 \\
245,996 \\
207,437\end{array}$ & $\begin{array}{l}08 \\
67 \\
66 \\
15\end{array}$ & $\begin{array}{l}217,706 \\
178,170 \\
497,009 \\
314,662 \\
217,052\end{array}$ & $\begin{array}{r}83,514 \\
48,305 \\
180,152 \\
106,228 \\
93,000\end{array}$ & $\begin{array}{l}13,965 \\
18,775 \\
40,218 \\
15,239 \\
10,801\end{array}$ & $\begin{array}{r}97,479 \\
67,080 \\
220,370 \\
121,467 \\
103,801\end{array}$ & $\begin{array}{l}19,014 \\
21,764 \\
28,397 \\
15,285 \\
14,000\end{array}$ & $\begin{array}{l}3 \\
2 \\
7 \\
4 \\
3\end{array}$ & $\begin{array}{l}66.70 \\
43.40 \\
55.77 \\
35.68 \\
19.37\end{array}$ & $\begin{array}{r}4,279,408 \\
10,660,586 \\
\ldots \ldots \ldots \%^{7} \\
17,246,660^{7}\end{array}$ & $\begin{array}{l}7.8 \\
2.5 \\
\ldots \\
1.9\end{array}$ & $\begin{array}{l}76 \\
77 \\
78 \\
79 \\
80\end{array}$ \\
\hline $\begin{array}{r}352,417 \\
221,160 \\
40,323 \\
78,600 \\
168,969\end{array}$ & $\begin{array}{r}28,667 \\
43,279 \\
3,202 \\
21,180 \\
20,322\end{array}$ & $\begin{array}{r}381,084 \\
264,439 \\
43,525 \\
99,780 \\
189,291\end{array}$ & $\begin{array}{r}149,340 \\
122,901 \\
26,777 \\
65,892 \\
86,377\end{array}$ & $\begin{array}{r}20,291 \\
17,504 \\
3,690 \\
10,000 \\
13,940\end{array}$ & $\begin{array}{r}169,631 \\
140,405 \\
30,467 \\
75,892 \\
100,317\end{array}$ & $\begin{array}{r}40,149 \\
32,081 \\
6,611 \\
8,400 \\
14,417\end{array}$ & $\begin{array}{r}590,864 \\
436,925 \\
80,603 \\
184,072 \\
304,025\end{array}$ & $\begin{array}{r}166.30 \\
36.99 \\
33.72 \\
102.78 \\
58.12\end{array}$ & $\begin{array}{r}11,863,775 \\
21,792,774 \\
3,357,688 \\
2,400,000 \\
\ldots \ldots \ldots{ }^{7}\end{array}$ & $\begin{array}{l}5.0 \\
2.0 \\
2.4 \\
7.7 \\
\cdots\end{array}$ & $\begin{array}{l}81 \\
82 \\
83 \\
84 \\
85\end{array}$ \\
\hline $\begin{array}{r}334,488 \\
129,948 \\
87,753 \\
39,895 \\
280,365\end{array}$ & $\begin{array}{r}24,420 \\
8,587 \\
21,319 \\
8,200 \\
47,651\end{array}$ & $\begin{array}{c}358,908 \\
187,660^{30} \\
109,072 \\
48,095 \\
328,016\end{array}$ & $\begin{array}{r}67,40 \dot{8}^{7} \\
55,365 \\
23,532 \\
111,384\end{array}$ & $\begin{array}{r}\ddot{14,915} \dot{7}^{7} \\
9,519 \\
4,863 \\
16,209\end{array}$ & $\begin{array}{r}163,880 \\
82,323 \\
64,884 \\
28,395 \\
127,593\end{array}$ & $\begin{array}{r}19,710 \\
13,646 \\
11,477 \\
3,888 \\
19,930\end{array}$ & & & $\begin{array}{r}18,194,945 \\
6,250,000 \\
2,806,560 \\
1,679,637 \\
10,723,455\end{array}$ & $\begin{array}{l}3.0 \\
4.5 \\
6.6 \\
4.8 \\
4.4\end{array}$ & $\begin{array}{l}88 \\
89 \\
90\end{array}$ \\
\hline $\begin{array}{l}133,505 \\
111,964 \\
168,985 \\
195,974 \\
210,954\end{array}$ & $\begin{array}{l}33,172 \\
16,754 \\
30,410 \\
20,913 \\
14,782\end{array}$ & $\begin{array}{l}166,677 \\
128,718 \\
199,395 \\
216,887 \\
225,736\end{array}$ & $\begin{array}{r}62,966 \\
64,694 \\
68,837 \\
86,859 \\
128,322\end{array}$ & $\begin{array}{r}7,500 \\
8,481 \\
10,425 \\
8,326 \\
18,429\end{array}$ & $\begin{array}{r}70,466 \\
73,175 \\
79,262 \\
95,185 \\
146,751\end{array}$ & $\begin{array}{r}16,400 \\
5,191 \\
6,775 \\
10,840 \\
18,344\end{array}$ & $\begin{array}{l}253,543 \\
207,084 \\
285,432 \\
322,912 \\
390,831\end{array}$ & $\begin{array}{l}45.65 \\
40.82 \\
29.69 \\
20.99 \\
41.39\end{array}$ & $\begin{array}{r}7,819,879 \\
3,660,295 \\
\ddot{8}, 471,99 \dot{5}^{7} \\
13,534,477\end{array}$ & $\begin{array}{l}3.2 \\
5.7 \\
3 . \dot{8} \\
2.9\end{array}$ & 91 \\
\hline $\begin{array}{r}278,520 \\
134,889 \\
93,876 \\
54,560 \\
74,882\end{array}$ & $\begin{array}{r}83,224 \\
13,413 \\
36,800 \\
29,440 \\
7,391\end{array}$ & $\begin{array}{r}361,744 \\
148,302 \\
130,676 \\
84,000 \\
82,273\end{array}$ & $\begin{array}{r}159,689 \\
91,251 \\
68,669 \\
22,194 \\
31,201\end{array}$ & $\begin{array}{r}18,426 \\
14,359 \\
17,792 \\
219 \\
6,020\end{array}$ & $\begin{array}{r}178,11 \\
105,61 \\
86,46 \\
22,41 \\
37,22\end{array}$ & $\begin{array}{r}25,082 \\
10,898 \\
21,584 \\
9,238 \\
5,852\end{array}$ & & $\begin{array}{l}32.34 \\
38.74 \\
28.71 \\
31.18 \\
42.58\end{array}$ & 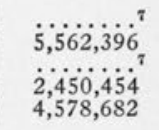 & $\begin{array}{l}\dot{4.8} \\
\dot{4} .7 \\
2.7\end{array}$ & $\begin{array}{r}96 \\
97 \\
98 \\
99 \\
100\end{array}$ \\
\hline $\begin{array}{l}258,618 \\
173,394 \\
454,895 \\
194,714 \\
363,131\end{array}$ & $\begin{array}{l}25,158 \\
36,623 \\
92,188 \\
28,028 \\
60,890\end{array}$ & $\begin{array}{l}283,776 \\
210,017 \\
547,083 \\
222,742 \\
424,021\end{array}$ & $\begin{array}{r}118,182 \\
112,343 \\
170,719 \\
66,012 \\
184,705\end{array}$ & $\begin{array}{l}19,447 \\
15,565 \\
18,333 \\
12,263 \\
17,547\end{array}$ & $\begin{array}{r}137,629 \\
127,908 \\
189,052 \\
78,275 \\
202,252\end{array}$ & $\begin{array}{l}34,782 \\
21,611 \\
42,492 \\
15,700 \\
36,032\end{array}$ & & $\begin{array}{r}112.09 \\
28.04 \\
54.97 \\
57.36 \\
35.80\end{array}$ & $\begin{array}{c}6,400,000^{16} \\
11,113,510 \\
15,767,912 \\
13,987,032^{16}\end{array}$ & $\begin{array}{l}7.1 \\
3.2 \\
4.9 \\
4.7\end{array}$ & $\begin{array}{l}103 \\
104 \\
105\end{array}$ \\
\hline $\begin{array}{r}146,820 \\
162,258 \\
450,635 \\
56,186 \\
741,382\end{array}$ & $\begin{array}{l}24,000 \\
30,540 \\
67,266 \\
11,561 \\
\ldots \ldots\end{array}$ & $\begin{array}{r}170,820 \\
192,798 \\
517,901 \\
67,747 \\
741,382\end{array}$ & $\begin{array}{r}53,867 \\
64,591 \\
166,026 \\
\ldots \ldots \ldots 7 \\
\ldots \ldots \ldots 7\end{array}$ & $\begin{array}{r}8,000 \\
13,750 \\
37,295 \\
\ldots \ldots \ldots\end{array}$ & $\begin{array}{r}61 \\
78, \\
203, \\
32, \\
480\end{array}$ & $\begin{array}{c}13,146 \\
13,582^{31} \\
41,825 \\
4,038 \\
\ldots \ldots 7^{7}\end{array}$ & $\begin{array}{c}245,833 \\
284,721 \\
763,047 \\
104,014 \\
1,221,877^{32}\end{array}$ & $\begin{array}{r}41.49 \\
36.87 \\
50.42 \\
38.27 \\
166.17\end{array}$ & $\begin{array}{c}6,720,000 \\
7,810,027 \\
26,632,861 \\
4,972,670 \\
22,533,759^{33}\end{array}$ & $\begin{array}{l}3.7 \\
3.6 \\
2.9 \\
2.1 \\
5.4\end{array}$ & $\begin{array}{l}106 \\
107 \\
108 \\
109\end{array}$ \\
\hline $\begin{array}{r}1,310,312 \\
165,548 \\
23,340 \\
110\end{array}$ & $\begin{array}{r}254,827 \\
21,180 \\
2,600 \\
105\end{array}$ & $\begin{array}{r}1,565,139 \\
189,291 \\
27,831 \\
110\end{array}$ & $\begin{array}{r}479,973 \\
73,794 \\
16,080 \\
104\end{array}$ & $\begin{array}{r}12,375 \\
219 \\
104\end{array}$ & $\begin{array}{r}94,646 \\
18,100 \\
110\end{array}$ & $\begin{array}{r}332,219 \\
13,646 \\
1,427 \\
109\end{array}$ & $\begin{array}{r}174,609 \\
292,119 \\
48,867 \\
110\end{array}$ & $\begin{array}{r}275.00 \\
41.10 \\
14.77 \\
110\end{array}$ & $\begin{array}{r}54,238,782 \\
7,769,029 \\
800,993 \\
94\end{array}$ & $\begin{array}{r}7.8 \\
3.7 \\
1.1 \\
94\end{array}$ & $\begin{array}{l}\operatorname{lgh} \\
\text { lian } \\
\mathrm{L}^{40} \\
\mathrm{~N}^{40}\end{array}$ \\
\hline
\end{tabular}

cine and Dentistry. 21. Excludes Branch College Libraries. 22. Excludes Law Library. 23. Includes $\$ 17,500$ nonsalaried service. 24. Included in staff salaries. 25 . Includes $\$ 1,500$ nonsalaried service. 26 . Off-campus binding only. 27. Includes heat, light and water. 28. Includes $\$ 300$ nonsalaried service. 29. Excludes College of Medicine and its Library. 30. Includes $\$ 49,125$ nonsalaried service. 31. Includes expenditures for social security and anForce personnel enrolled in residence centers on six. 33. Includes dormitory costs. 34. Includes 1,092 Air Atlantic programs not included. 36. Includes administrative costs on $\$ 34,000,000$ defense contracts. 37 . Evanston campus only. 38. Includes off-campus extension students. 39. Excludes government contracts. 40. Number of libraries on which high, median and low are based. 41. In computing this figure, off-campus extension students have been omitted. 42. Includes expenditures for the University Hospital. 43 . Includes 9,835 part-time students in the Evening Division, Chicago Campus.

\section{Statistics， I 955-I956 (Group I)}

\begin{tabular}{|c|c|c|c|c|c|c|c|c|c|c|}
\hline $\begin{array}{l}\text { ALL O } \\
\text { SIONA }\end{array}$ & $\begin{array}{l}\text { THER P } \\
\text { L AsSIS? }\end{array}$ & $\begin{array}{l}\text { ROFES- } \\
\text { ANTS }\end{array}$ & ALL No & $\begin{array}{l}\text { ONPROFE: } \\
\text { ONSISTAN }\end{array}$ & SSIONAL & Total & $\begin{array}{l}\text { MBER OF I } \\
\text { TIME EQU }\end{array}$ & $\begin{array}{l}\text { LOYEES } \\
\text { LENT }^{2}\end{array}$ & $\begin{array}{l}\text { Working } \\
\text { Hours in }\end{array}$ & Hours \\
\hline $\begin{array}{l}\text { Mini- } \\
\text { mum }\end{array}$ & $\begin{array}{l}\text { Maxi- } \\
\text { mum }\end{array}$ & $\begin{array}{c}\text { Num- } \\
\text { ber }\end{array}$ & $\begin{array}{l}\text { Mini- } \\
\text { mum }\end{array}$ & $\begin{array}{l}\text { Maxi- } \\
\text { mum }\end{array}$ & $\underset{\text { ber }}{\text { Num. }}$ & $\begin{array}{c}\text { Pro- } \\
\text { fessional }\end{array}$ & $\begin{array}{l}\text { Nonpro- } \\
\text { fessional }\end{array}$ & Total & $\begin{array}{l}\text { Fiscal } \\
\text { Year }^{3}\end{array}$ & $\begin{array}{l}\text { dent As- } \\
\text { sistance }\end{array}$ \\
\hline $\begin{array}{c}\$ 2,700 \\
3,480 \\
3,600 \\
2,800 \\
\ldots \ldots\end{array}$ & $\begin{array}{l}4,000 \\
4,500 \\
4,300 \\
3,950 \\
\ldots . .4\end{array}$ & $\begin{array}{l}28-1 / 2 \\
10 \\
7 \\
9-1 / 2 \\
.^{4}\end{array}$ & $\begin{array}{r}\$ 1,700 \\
2,160 \\
2,525 \\
1,620 \\
\ldots \ldots\end{array}$ & $\begin{array}{r}\$ 2,700 \\
2,700 \\
3,600 \\
2,100 \\
\ldots \ldots\end{array}$ & $\begin{array}{l}14 \\
14-1 / 2 \\
12 \\
12 \\
\ldots .4\end{array}$ & $\begin{array}{l}38-1 / 2 \\
15 \\
13-1 / 2 \\
17-1 / 4 \\
14-1 / 2\end{array}$ & $\begin{array}{l}14 \\
14-1 / 2 \\
10 \\
12 \\
7-1 / 2\end{array}$ & $\begin{array}{l}52-1 / 2 \\
29-1 / 2 \\
23-1 / 2 \\
29-1 / 4 \\
22\end{array}$ & $\begin{array}{l}1,800 \\
1,850 \\
1,824 \\
1,856 \\
1,840\end{array}$ & $\begin{array}{l}50,000 \\
15,000^{\circ} \\
26,176 \\
22,868 \\
54,045\end{array}$ \\
\hline $\begin{array}{l}3,200 \\
3,100 \\
4,100 \\
3,000\end{array}$ & $\begin{array}{l}5,300 \\
4,300 \\
6,200 \\
4,080\end{array}$ & $\begin{array}{l}11 \\
19^{4} \\
13 \\
14\end{array}$ & $\begin{array}{l}2,200 \\
1, \ddot{8} 7{ }^{4} \\
3,250 \\
2,040\end{array}$ & $\begin{array}{l}3,200 \\
3,4^{4} \\
3,840 \\
3,000\end{array}$ & $\begin{array}{l}15 \\
40^{4} \\
10 \\
43\end{array}$ & $\begin{array}{l}20 \\
40 \\
26 \\
25-1 / 2 \\
24-3 / 38\end{array}$ & $\begin{array}{l}15 \\
42 \\
40 \\
20 \\
41-1 / 19\end{array}$ & $\begin{array}{l}35 \\
82 \\
66 \\
45-1 / 2 \\
65-5 / 38\end{array}$ & $\begin{array}{l}1,840 \\
1,624 \\
1,820 \\
1,400 \\
1,710\end{array}$ & $\begin{array}{l}15,000 \\
37,000^{\circ} \\
10,187 \\
22,941 \\
23,200\end{array}$ \\
\hline $\begin{array}{l}1,800^{9} \\
4,092 \\
4,092 \\
4,092 \\
4,092\end{array}$ & $\begin{array}{l}4,795 \\
7,728 \\
6,060 \\
7,356 \\
4,980\end{array}$ & $\begin{array}{c}5 \\
100-3 / 4 \\
9 \\
67 \\
3\end{array}$ & $\begin{array}{l}1,080^{\circ} \\
2,640 \\
2,640 \\
2,640 \\
2,640\end{array}$ & $\begin{array}{l}3,530 \\
6,840 \\
4,512 \\
6,672 \\
4,512\end{array}$ & $\begin{array}{l}25 \\
182-1 / 2 \\
19 \\
97 \\
11-1 / 2\end{array}$ & $\begin{array}{l}12-1 / 2 \\
131-1 / 4 \\
16 \\
85 \\
10\end{array}$ & $\begin{array}{c}23 \\
178-4 / 5 \\
17-1 / 2 \\
90 \\
11-1 / 2\end{array}$ & $\begin{array}{c}35-1 / 2 \\
310-1 / 20 \\
33-1 / 2 \\
175^{12} \\
21-1 / 2\end{array}$ & $\begin{array}{l}1,617 \\
1,824 \\
1,816 \\
1,824 \\
1,840\end{array}$ & $\begin{array}{c}8,222 \\
186,453 \\
8,300^{6} \\
167,728 \\
13,333\end{array}$ \\
\hline
\end{tabular}


College and University Library

\section{Associate or \\ Assistant \\ Chief Librarian \\ Library $^{1}$

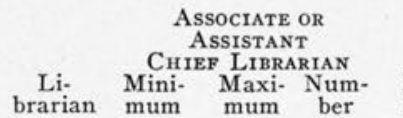 \\ Department Heads \\ mum mum}

16. California (Santa Barbara).

17. California Inst. of Tech. .

18. Catholic U. of America $\ldots \ldots$

20. Cincinnati $\ldots \ldots \ldots \ldots \ldots \ldots$

21. City College (N.Y.) .....

22. Clark Colorado $^{15} \ldots \ldots \ldots \ldots \ldots \ldots \ldots \ldots \ldots$

24. Colorado A. \& $\mathrm{M} . \ldots \ldots \ldots$

25. Columbia ${ }^{17} \ldots \ldots \ldots \ldots \ldots$

26. Cornell

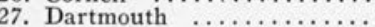

28. Delaware $\ldots \ldots \ldots \ldots \ldots \ldots$

29. Denver

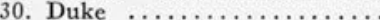

31. Florida

32. Florida state $\ldots \ldots \ldots \ldots \ldots \ldots$

33. Fordham $\ldots \ldots \ldots \ldots \ldots \ldots \ldots$

35. Georgia $\ldots \ldots \ldots$

36. Harvard

37. Hawaii ${ }^{2}$

38. Houston

39. Hunter

40. Illinois

41. Indian

42. Iowa

43. Iowa State $: \ldots \ldots \ldots \ldots \ldots$

45. Johns Hopkins $\ldots \ldots \ldots \ldots \ldots$

46. Kansas $\quad \ldots \ldots \ldots \ldots \ldots \ldots \ldots$

48. Kentucky $\ldots \ldots \ldots$

49. Lehigh ...................

51. Maine

an.

53. Massachusetts

54. Mass, Inst, of Tech $\ldots$

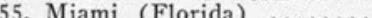

56. Michigan 2

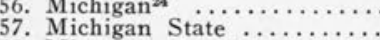

58. Minnesota

59. Mississippi ${ }^{25}{ }^{\cdots} \ldots \ldots \ldots \ldots \ldots$

60. Mississippi State ........

61. Missouri $\ldots \ldots \ldots \ldots \ldots \ldots$

62. Montana.$\ldots \ldots \ldots \ldots \ldots$

63. Montana State...$\ldots \ldots \ldots$

65. Nebraska

66. New Hampshire .........

67. New Hamps

68. New Yexico ............

69. North Carolina

70. North Carolina State ........

71. North Dakota ..........

72. Northwestern $\quad . \ldots \ldots \ldots \ldots$

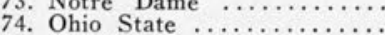

75. Oklahoma $\ldots \ldots \ldots \ldots \ldots \ldots \ldots \ldots$

76. Oregon ${ }^{27}$

77. Oregon State

79. Pennsylvania State $\ldots . . . .$.

80. Pittsburgh

81. Princeton $\ldots \ldots \ldots \ldots \ldots \ldots$ 82. Purdue

83. Rhode Island $\ldots \ldots \ldots \ldots \ldots$

84. Rice $\ldots \ldots \ldots \ldots \ldots \ldots \ldots \ldots$

86. Rutgers

87. Saint Louis

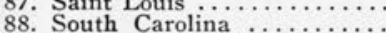

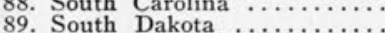

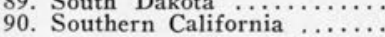

$\begin{array}{llll}9,384 & 6,060 & 7,728 & 2 \\ \cdots \ldots{ }^{4} & 6,000^{7} & 8,400^{8} & 1 \\ 6,600 & 4,700 & \ldots \ldots & 1 \\ \cdots \cdots & \cdots \cdots & \cdots \cdots & 2 \\ \ldots \ldots & \ldots \ldots & \ldots \ldots & \ldots\end{array}$

$11,850 \quad 7,650$

8,650

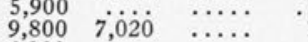

$6,900, \ldots \cdots, \ldots \cdots$.

$\cdots \cdots 4_{4}^{4} 6,025,10,000 \quad 8^{10}$

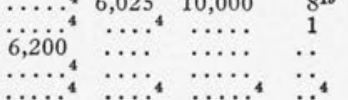

$6,740-6,360$

$3,600 \quad 5,400$

$3,600 \quad 4,500 \quad 8$

$5,0 \dot{\sigma}^{4} \quad \dddot{6}, 2 \dot{\varphi}^{4} \quad 12$

$6,150 \quad 7,150 \quad 10$

4,000
$4,020 \quad 3,640 \quad 9$

$4,120^{16} \quad \ldots \cdots, \quad 1$

$4,700 \quad \ldots \ldots 4^{4} \quad 43$

$\begin{array}{rrr}4,176 & 6,120 & 16 \\ 3,900 & 6,000 & 7\end{array}$

$3,900 \quad 6,000$

$4,818 \quad 4,830$

$9,300 \quad 7,300$

$9,200 \quad 5,700$

$7,800 \quad 5,000$

$9,200 \quad 6,500$

$\ddot{7}, \dot{200}$

6,000

$9,300 \quad 6,800$

$\cdots \cdots$

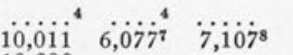

10,200
9,750 $\ddot{7,050} \quad \ddot{8,650}$

$\begin{array}{rrr}15,500 & 7,600 & 10,800\end{array}$

$\ddot{11,700} 8,00^{4} \quad \ldots \ldots$.

$10,500,7,680$

$\cdots \cdots^{4} \quad \cdots{ }^{4} \quad \cdots \cdots^{4}$

$10,500 \quad 6,300$

$7,920 \quad 5,520$

$\ldots \ldots 4$

$\ddot{10,008} \quad \ddot{0,000} \quad \ddot{9,000}{ }^{4} \quad \ddot{3}^{4}$

7,400

$7,500000_{4} \quad 3,960$

$11,000 \quad \ldots *^{4} \quad \ldots *^{4} \quad{ }^{4}$

$110000^{4} \quad \ddot{7} 00^{4} \quad \ddot{9}, 000^{4}$

$12,000 \quad 9,000$

$\ddot{7}, 20$

8,800

$7,300-6,500$

$6,600 \quad 5,500$

$9,200 \quad 5,900$

10,200

$6,500,5,700$

$\cdots \cdots 4, \ldots 4^{4}$

$\ddot{10,000} \quad 5,3 \dot{3} \dot{2}$

$\begin{array}{ll}8,120 & \cdots \\ 7,600 & 5,000\end{array}$

$11,000,7,100$

$\ddot{11,004} 8, \ddot{1} 9 \dot{6}, \quad \ddot{8,604}$

10,972

$10,972^{29} \ldots$

... 6,800

$9,120 \quad 7,188$

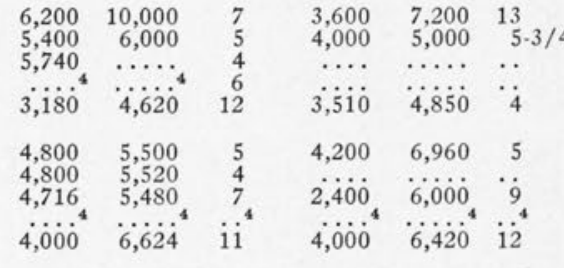

Head Librarian

School, College,

AND DEPART MENTAL

mum maxi- Num

$\because \cdots^{4} \ldots 0^{4} \quad \ldots \ldots \quad 1 \quad 5,750$

5,750
5,300

4,400

$\cdots \cdots+5,240 \quad \ldots .$.

$8,900,5,200 \quad \ldots \ldots$

$\begin{array}{ccc}\cdots & \cdots & \cdots\end{array}$

$\begin{array}{lll}10,860 & 6,534^{7} & 7,854^{8}\end{array}$

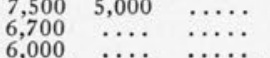

$\begin{array}{lll}6,000 & \ldots & \ldots \\ \cdots & \ldots & \ldots\end{array}$ $\begin{array}{lll}5,478 & 6,798 & 11 \\ 3,120 & 5,400 & \ldots \\ 3,600 & 3,800 & 4^{40} \\ 4,400 & 4,900 & 2 \\ \ldots \ldots & \ldots \ldots & 6\end{array}$

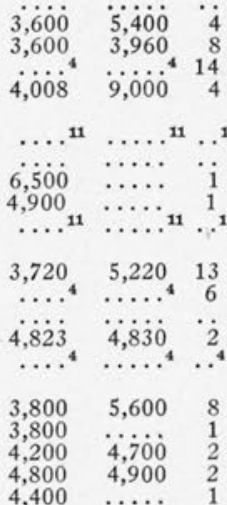

$\begin{array}{lll}3,500 & 14,000 & 18\end{array}$

$\begin{array}{lll}5,871^{7} & 7,298^{8} & 1 \\ 3,960^{16} & 6,360 & 3\end{array}$

$\ddot{4,400} \quad \ddot{8} \ddot{5} \dot{0} 0 \dot{30}$

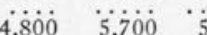

$4,100 \quad 7,000$

$4,668 \quad 14,000 \quad 20-33 / 50$

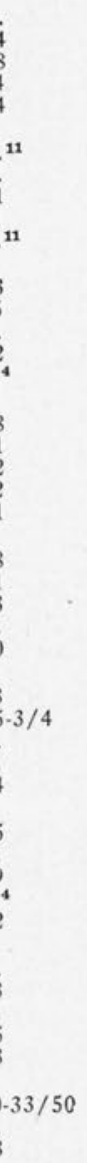

2

26

$\cdots 45$

$3,456 \quad 7,800 \quad 8$

$4,750^{28}$

$4,200 \quad 6,096 \quad 11$

$3,540 \quad 5,040 \quad 12$

$\begin{array}{rrr}3,600 & 7,000 \quad 6\end{array}$

$\ddot{3,3000} \ddot{5, j} \dot{5} 00 \quad \ddot{3}$

$5,478 \quad 9,570 \quad 9$

$4,900, \quad 3,500, \quad 2$ 
Salary Statistics, ${ }^{1955-56}$ (Group I, Cont.)

\begin{tabular}{|c|c|c|c|c|c|c|c|c|c|c|}
\hline \multicolumn{3}{|c|}{ All Other Profes- } & \multicolumn{3}{|c|}{$\begin{array}{c}\text { All Nonprofessional } \\
\text { Assistants }\end{array}$} & \multicolumn{3}{|c|}{$\begin{array}{l}\text { TOTAL NUMBER OF EMPLOYEES } \\
\text { IN FULL-TIME EQUIVALENT }{ }^{2}\end{array}$} & \multirow{2}{*}{$\begin{array}{c}\text { Working } \\
\text { Hours in } \\
\text { Fiscal } \\
\text { Year }^{3}\end{array}$} & \multirow{2}{*}{$\begin{array}{l}\text { Hours } \\
\text { of Stu- } \\
\text { dent As- } \\
\text { sistance }\end{array}$} \\
\hline $\begin{array}{l}\text { Mini- } \\
\text { mum }\end{array}$ & Maxi- & $\begin{array}{l}\text { Num- } \\
\text { ber }\end{array}$ & $\begin{array}{l}\text { Mini- } \\
\text { mum }\end{array}$ & $\begin{array}{l}\text { Maxi- } \\
\text { mum }\end{array}$ & Num- & $\begin{array}{l}\text { Pro- } \\
\text { fessional }\end{array}$ & $\begin{array}{l}\text { Nonpro- } \\
\text { fessional }\end{array}$ & Total & & \\
\hline $\begin{array}{l}4,092 \\
\ddot{3,300} \\
\dot{4,0} \ddot{5}^{4}\end{array}$ & $\begin{array}{l}4,980 \\
\ddot{3}, 900 \\
\ddot{4}, 9 \dot{4}^{4}\end{array}$ & $\begin{array}{r}8 \\
77 \\
26 \\
8\end{array}$ & $\begin{array}{l}2,916 \\
2,400 \\
2,100 \\
2,304\end{array}$ & $\begin{array}{l}3,624 \\
4,080 \\
3,900 \\
3,804\end{array}$ & $\begin{array}{l}11 \\
12-1 / 4 \\
14 \\
82 \\
30-1 / 2\end{array}$ & $\begin{array}{l}13 \\
8-1 / 4 \\
20-1 / 2^{13} \\
51 \\
17\end{array}$ & $\begin{array}{l}11 \\
12-1 / 4 \\
20^{14} \\
71 \\
30-1 / 2\end{array}$ & $\begin{array}{l}24 \\
20-1 / 2 \\
40-1 / 2 \\
122 \\
47-1 / 2\end{array}$ & $\begin{array}{l}1,824 \\
1,944 \\
1,764 \\
1,751 \\
1,600^{6}\end{array}$ & $\begin{array}{r}7,548 \\
2,120 \\
16,598 \\
105,615 \\
21,500^{6}\end{array}$ \\
\hline $\begin{array}{l}4,850 \\
3,800 \\
3,540 \\
3,700 \\
3,800\end{array}$ & $\begin{array}{l}5,900 \\
4,560 \\
5,200 \\
4,850\end{array}$ & $\begin{array}{l}30 \\
1 \\
10 \\
7 \\
48-1 / 2\end{array}$ & $\begin{array}{l}2,020^{9} \\
2,080 \\
2,040 \\
2,568 \\
2,000\end{array}$ & $\begin{array}{l}3,450 \\
2,236 \\
4,020 \\
3,276 \\
3,900\end{array}$ & $\begin{array}{r}16 \\
6 \\
22 \\
11 \\
225\end{array}$ & $\begin{array}{l}49 \\
3 \\
20 \\
9-2 / 3 \\
95\end{array}$ & $\begin{array}{l}14-3 / 4 \\
6 \\
21 \\
10-1 / 2 \\
218\end{array}$ & $\begin{array}{l}63-3 / 4 \\
9 \\
41 \\
20-1 / 6 \\
313\end{array}$ & $\begin{array}{l}1,455 \\
1,645 \\
1,840 \\
1,892 \\
1,757\end{array}$ & $\begin{array}{c}45,947 \\
5,614 \\
45,290 \\
16,258 \\
\ldots \ldots{ }^{18}\end{array}$ \\
\hline $\begin{array}{l}3,720 \\
3,450 \\
3,900 \\
3,640 \\
3,300\end{array}$ & $\begin{array}{l}7,500 \\
4,800 \\
3,900 \\
4,100 \\
4,500\end{array}$ & $\begin{array}{r}54 \\
12 \\
4 \\
8 \\
28\end{array}$ & $\begin{array}{l}2,460 \\
1,800 \\
2,100 \\
2,160 \\
1,800\end{array}$ & $\begin{array}{l}4,176 \\
4,950 \\
3,000 \\
3,204 \\
3,400\end{array}$ & $\begin{array}{l}94 \\
40^{20} \\
16 \\
18 \\
43\end{array}$ & $\begin{array}{l}86-2 / 3 \\
25 \\
9-1 / 2 \\
15 \\
38\end{array}$ & $\begin{array}{l}96 \\
41^{20} \\
13-1 / 2 \\
17 \\
43-1 / 4\end{array}$ & $\begin{array}{l}182-2 / 3 \\
66^{20} \\
23 \\
32 \\
81-1 / 4\end{array}$ & $\begin{array}{l}1,833 \\
1,648 \\
1,700 \\
1,717 \\
1,840\end{array}$ & $\begin{array}{r}127,701 \\
15,415 \\
4,875 \\
14,150 \\
51,868\end{array}$ \\
\hline $\begin{array}{l}3,600 \\
3,400 \\
3,800 \\
3,700 \\
3,700\end{array}$ & $\begin{array}{l}4,950 \\
4,650 \\
4,200 \\
4,600 \\
4,500\end{array}$ & $\begin{array}{l}26 \\
24 \\
13 \\
15 \\
14\end{array}$ & $\begin{array}{l}1,900 \\
2,160 \\
2,444 \\
2,120 \\
2,400\end{array}$ & $\begin{array}{l}3,900 \\
3,331 \\
3,200 \\
2,820 \\
3,600\end{array}$ & $\begin{array}{l}59-1 / 2 \\
26 \\
22 \\
28 \\
16\end{array}$ & $\begin{array}{l}42 \\
34 \\
21 \\
25 \\
21-3 / 4\end{array}$ & $\begin{array}{l}58-1 / 2 \\
26 \\
18 \\
26-1 / 2 \\
16\end{array}$ & $\begin{array}{l}100-1 / 2 \\
60 \\
39 \\
51-1 / 2 \\
37-3 / 4\end{array}$ & $\begin{array}{l}1,810 \\
1,800 \\
1,652 \\
1,700 \\
1,802\end{array}$ & $\begin{array}{l}71,681 \\
11,620 \\
17,617 \\
12,406\end{array}$ \\
\hline $\begin{array}{l}3,240 \\
3,553 \\
3,225 \\
4,100 \\
3,900\end{array}$ & $\begin{array}{r}10,000 \\
5,098 \\
4,560 \\
5,900 \\
7,200\end{array}$ & $\begin{array}{l}81 \\
12 \\
6 \\
13 \\
96-1 / 2\end{array}$ & $\begin{array}{l}1,980 \\
2,101 \\
2,040 \\
3,150 \\
1,920\end{array}$ & $\begin{array}{l}6,000 \\
3,708 \\
3,780 \\
4,450 \\
4,800\end{array}$ & $\begin{array}{c}251 \\
16 \\
17 \\
9 \\
118-1 / 2\end{array}$ & $\begin{array}{c}135 \\
18 \\
18-1 / 2 \\
20-14 / 25 \\
134-1 / 2\end{array}$ & $\begin{array}{l}251 \\
16 \\
21 \\
12 \\
117-1 / 2\end{array}$ & $\begin{array}{l}386 \\
34 \\
39-1 / 2 \\
32-14 / 25 \\
252\end{array}$ & $\begin{array}{l}1,610 \\
1,848 \\
1,748 \\
1,445 \\
1,800\end{array}$ & $\begin{array}{r}70,000^{6} \\
28,720 \\
34,201 \\
28,090 \\
100,910\end{array}$ \\
\hline $\begin{array}{l}3,900 \\
3,200^{\circ} \\
3,840 \\
1,980^{\circ}\end{array}$ & $\begin{array}{l}5,500 \\
5,200 \\
4,680 \\
3,420\end{array}$ & $\begin{array}{l}22 \\
18 \\
14 \\
13-1 / 2 \\
10\end{array}$ & $\begin{array}{l}2,400 \\
2,160 \\
1,920 \\
2,100^{4}\end{array}$ & $\begin{array}{l}4,000 \\
3,780 \\
3,540 \\
\because 300^{4}\end{array}$ & $\begin{array}{l}54 \\
47-3 / 4 \\
28 \\
48 \\
13\end{array}$ & $\begin{array}{l}44 \\
30-3 / 4 \\
18-1 / 2 \\
23 \\
26-1 / 2\end{array}$ & $\begin{array}{l}54 \\
48-1 / 4 \\
28 \\
47-1 / 2 \\
12-1 / 2\end{array}$ & $\begin{array}{l}98 \\
79 \\
46-1 / 2 \\
70-1 / 2 \\
39\end{array}$ & $\begin{array}{l}1,750^{6} \\
1,848 \\
1,848 \\
1,702 \\
1,880\end{array}$ & $\begin{array}{l}\dddot{49,306} \\
23,578 \\
19,973 \\
62,666\end{array}$ \\
\hline $\begin{array}{l}3,700 \\
3,840 \\
3,080 \\
3,400^{4}\end{array}$ & $\begin{array}{l}6,300 \\
4,680 \\
4,472 \\
\div, 25 \dot{6}^{4}\end{array}$ & $\begin{array}{l}28 \\
12 \\
18 \\
20\end{array}$ & $\begin{array}{l}1,732 \\
1,980 \\
1,900 \\
2,004^{4}\end{array}$ & $\begin{array}{l}5,200 \\
2,520 \\
2,856 \\
3,420\end{array}$ & $\begin{array}{l}38 \\
8 \\
23-1 / 2 \\
32^{4}\end{array}$ & $\begin{array}{l}41-1 / 4 \\
18 \\
29 \\
9 \\
47\end{array}$ & $\begin{array}{l}35 \\
8 \\
28-1 / 2 \\
6-1 / 2 \\
32\end{array}$ & $\begin{array}{l}76-1 / 4 \\
26 \\
57-1 / 2 \\
15-1 / 2 \\
79\end{array}$ & $\begin{array}{l}1,856 \\
1,852^{4} \\
1,864 \\
\cdots \cdots\end{array}$ & $\begin{array}{c}88,464 \\
24,610 \\
28,000^{6} \\
8,154 \\
34,753\end{array}$ \\
\hline $\begin{array}{l}3,400 \\
3,572\end{array}$ & $\begin{array}{l}4,400 \\
4,550\end{array}$ & $\begin{array}{r}3 \\
19\end{array}$ & $\begin{array}{l}1,976 \\
2,690 \\
2,640\end{array}$ & $\begin{array}{l}2,808 \\
3,225 \\
3,840\end{array}$ & $\begin{array}{l}5 \\
24-1 / 2 \\
12\end{array}$ & $26^{7 \cdot 1 / 2}$ & $\begin{array}{r}6-1 / 2 \\
21-1 / 2 \\
13\end{array}$ & $\begin{array}{l}14 \\
47 \cdot 1 / 2 \\
15\end{array}$ & & $\begin{array}{r}9,235 \\
36,121 \\
6,140\end{array}$ \\
\hline $\begin{array}{l}3,020 \\
4,000\end{array}$ & $\begin{array}{l}4,600 \\
5,800\end{array}$ & $\begin{array}{l}14 \\
15\end{array}$ & $\begin{array}{l}2,640 \\
2,400 \\
1,920\end{array}$ & $\begin{array}{l}3,840 \\
3,780 \\
3,600\end{array}$ & & $\begin{array}{l}26 \\
23\end{array}$ & $\begin{array}{l}13 \\
33 \\
44\end{array}$ & $\begin{array}{l}15 \\
59 \\
67\end{array}$ & $\begin{array}{l}1,800 \\
1,610 \\
1,828\end{array}$ & $\begin{array}{r}6,140 \\
18,180 \\
24,240\end{array}$ \\
\hline $\begin{array}{l}4,000 \\
4,080 \\
3,792 \\
2,880 \\
2,700\end{array}$ & $\begin{array}{l}7,200 \\
5,120 \\
6,000 \\
3,600 \\
4,000\end{array}$ & $\begin{array}{r}90 \\
21 \\
67 \\
5 \\
5\end{array}$ & $\begin{array}{l}2,520 \\
2,662 \\
2,100 \\
1,560 \\
1,750\end{array}$ & $\begin{array}{l}5,520 \\
3,840 \\
3,948 \\
2,400 \\
2,700\end{array}$ & $\begin{array}{l}96-1 / 2 \\
35 \\
46 \\
9 \\
7\end{array}$ & $\begin{array}{l}119 \cdot 13 / 50 \\
36 \\
96 \\
11 \\
10\end{array}$ & $\begin{array}{l}62-11 / 20 \\
33 \\
48 \\
9 \\
9\end{array}$ & $\begin{array}{l}181-81 / 100 \\
69 \\
144 \\
20 \\
19\end{array}$ & $\begin{array}{l}1,840 \\
1,856 \\
1,904^{33} \\
1,856 \\
1,933\end{array}$ & $\begin{array}{r}114,777 \\
70,800 \\
106,365 \\
19,450 \\
14,219\end{array}$ \\
\hline $\begin{array}{l}3,400 \\
3,000 \\
3,400 \\
3,700 \\
5,200\end{array}$ & $\begin{array}{l}4,800 \\
4,100 \\
4,800 \\
5,200 \\
\ldots \ldots\end{array}$ & $\begin{array}{c}12-1 / 2 \\
6 \\
5-1 / 2 \\
23 \\
1\end{array}$ & $\begin{array}{l}1,680 \\
2,100 \\
2,400 \\
1,800 \\
3,000\end{array}$ & $\begin{array}{l}2,900 \\
2,280 \\
2,700 \\
4,300 \\
3,400\end{array}$ & $\begin{array}{l}30 \\
5 \\
4 \\
41 \\
5-3 / 4\end{array}$ & $\begin{array}{l}20-1 / 2 \\
12 \\
9-1 / 2 \\
34 \\
6\end{array}$ & $\begin{array}{l}31-1 / 2 \\
5 \\
4 \\
35 \\
3-3 / 4\end{array}$ & $\begin{array}{l}52 \\
17 \\
13-1 / 2 \\
69 \\
9-3 / 4\end{array}$ & $\begin{array}{l}1,832 \\
1,748 \\
1,724 \\
1,908 \\
1,754\end{array}$ & $\begin{array}{r}32,809 \\
7,943 \\
7,200 \\
50,532 \\
5,670\end{array}$ \\
\hline $\begin{array}{l}3,500 \\
4,200 \\
3,600 \\
3,638 \\
3,508\end{array}$ & $\begin{array}{l}4,925 \\
5,500 \\
5,288 \\
4,035\end{array}$ & $\begin{array}{l}1 \\
6 \\
33-2 / 3 \\
24 \\
3\end{array}$ & $\begin{array}{l}1,900 \\
2,400 \\
2,220 \\
2,100 \\
2,400\end{array}$ & $\begin{array}{l}3,000 \\
2,775 \\
3,600 \\
4,406 \\
3,254\end{array}$ & $\begin{array}{l}9 \\
11-1 / 2 \\
74-1 / 2 \\
45-1 / 4 \\
15\end{array}$ & $\begin{array}{l}11 \\
13 \\
42-2 / 3 \\
42-3 / 4 \\
12\end{array}$ & $\begin{array}{l}8 \\
10-1 / 2 \\
84-1 / 2 \\
45-1 / 4 \\
15\end{array}$ & $\begin{array}{l}19 \\
23-1 / 2 \\
127-1 / 6 \\
88 \\
27\end{array}$ & $\begin{array}{l}1,848 \\
1,787 \\
1,750^{6} \\
1,850 \\
1,794\end{array}$ & $\begin{array}{l}20,179 \\
24,555 \\
38,000^{6} \\
45,994 \\
11,351\end{array}$ \\
\hline $\begin{array}{l}4,000 \\
3,800 \\
3,600 \\
3,636 \\
3,456\end{array}$ & $\begin{array}{l}4,400 \\
5,000 \\
4,830 \\
5,844 \\
4,620\end{array}$ & $\begin{array}{l}2 \\
12^{26} \\
16 \\
47 \\
15-1 / 2\end{array}$ & $\begin{array}{l}1,920 \\
2,280 \\
1,800 \\
2,220 \\
1,920\end{array}$ & $\begin{array}{l}2,400 \\
3,360 \\
2,880 \\
6,000 \\
3,120\end{array}$ & $\begin{array}{l}3 \\
39^{26} \\
23 \\
86-1 / 2 \\
26-3 / 4\end{array}$ & $\begin{array}{l}8 \\
47 \\
26 \\
68 \\
31-1 / 2\end{array}$ & $\begin{array}{l}3 \\
52-1 / 2 \\
23 \\
86-1 / 2 \\
24-1 / 4\end{array}$ & $\begin{array}{l}11 \\
99-1 / 2 \\
49 \\
154-1 / 2 \\
55-3 / 4\end{array}$ & & \\
\hline $\begin{array}{l}3,586 \\
3,736 \\
3,500 \\
3,696 \\
3,300\end{array}$ & $\begin{array}{l}4,736 \\
5,105 \\
6,000 \\
4,800 \\
4,200\end{array}$ & $\begin{array}{l}18 \\
17 \\
30 \\
24 \\
19\end{array}$ & $\begin{array}{l}1,992 \\
2,075 \\
1,820 \\
1,920 \\
1,500\end{array}$ & $\begin{array}{l}3,540 \\
3,700 \\
4,000 \\
3,744 \\
2,970\end{array}$ & $\begin{array}{l}28-1 / 2 \\
18 \\
97 \\
43 \\
42\end{array}$ & $\begin{array}{l}30 \\
24-19 / 50 \\
56-1 / 4 \\
36 \\
30\end{array}$ & $\begin{array}{l}28-1 / 2 \\
16-7 / 20 \\
94-1 / 4 \\
36 \\
42\end{array}$ & $\begin{array}{l}58-1 / 2 \\
40-73 / 100 \\
150-1 / 2 \\
72 \\
72\end{array}$ & $\begin{array}{l}1,880 \\
1,547^{4} \\
1,816 \\
1,725\end{array}$ & $\begin{array}{l}19,376 \\
27,031 \\
29,780 \\
75,943 \\
15,002\end{array}$ \\
\hline $\begin{array}{l}3,300 \\
4,600 \\
3,400 \\
3,100 \\
3,500\end{array}$ & $\begin{array}{l}4,500 \\
4,800 \\
4,410 \\
3,800 \\
5,200\end{array}$ & $\begin{array}{c}22 \\
7 \\
5 \\
9 \\
16-1 / 2\end{array}$ & $\begin{array}{l}1,920 \\
2,280 \\
1,980 \\
2,600 \\
2,340\end{array}$ & $\begin{array}{l}3,600 \\
4,140 \\
2,840 \\
3,000 \\
3,224\end{array}$ & $\begin{array}{r}99 \\
48 \\
4 \\
6 \\
27\end{array}$ & $\begin{array}{l}36-5 / 6 \\
23 \\
7 \\
15-1 / 2 \\
26-1 / 2\end{array}$ & $\begin{array}{l}88-1 / 3 \\
46 \\
4 \\
5-1 / 2 \\
27\end{array}$ & $\begin{array}{l}125-1 / 6 \\
69 \\
11 \\
21 \\
53-1 / 2\end{array}$ & $\begin{array}{l}1,885 \\
1,816 \\
1,710 \\
1,840 \\
1,617\end{array}$ & $\begin{array}{c}17,000^{6} \\
51,203 \\
5,300 \\
25,596 \\
29,050\end{array}$ \\
\hline $\begin{array}{l}3,432 \\
3,000 \\
3,200 \\
3,200 \\
\ldots \ldots\end{array}$ & $\begin{array}{l}5,412 \\
4,200 \\
3,500 \\
\ldots \ldots 4\end{array}$ & $\begin{array}{l}21 \\
3^{4} \\
3^{30} \\
27\end{array}$ & $\begin{array}{l}2,400 \\
2,160 \\
2,100 \\
2,400 \\
\ldots \ldots\end{array}$ & $\begin{array}{c}3,600 \\
3,600 \\
2,400 \\
2,575 \\
\ldots \ldots\end{array}$ & $\begin{array}{l}40 \\
14^{4} \\
3 \\
48\end{array}$ & $\begin{array}{l}42 \\
27 \\
10-1 / 2 \\
8 \\
43-1 / 2\end{array}$ & $\begin{array}{l}40 \\
25 \\
23 \\
5-1 / 2 \\
44-3 / 5\end{array}$ & $\begin{array}{l}82 \\
52 \\
33-1 / 2 \\
13-1 / 2 \\
88-1 / 10\end{array}$ & $\begin{array}{l}1,828 \\
1,672 \\
1,764 \\
2,121 \\
1,840\end{array}$ & $\begin{array}{c}26,862 \\
9,541 \\
25,000^{6} \\
6,769 \\
62,487\end{array}$ \\
\hline
\end{tabular}




\begin{tabular}{|c|c|c|c|c|c|c|c|c|c|c|}
\hline \multirow[b]{2}{*}{ Library $^{1}$} & \multirow[b]{2}{*}{$\underset{\text { brarian }}{\text { Li- }}$} & \multicolumn{3}{|c|}{$\begin{array}{c}\text { Associate or } \\
\text { Assistant } \\
\text { CHIEF LiBRARIAN }\end{array}$} & \multicolumn{3}{|c|}{ Department Heads } & \multicolumn{3}{|c|}{$\begin{array}{l}\text { HEAD LIBRARIAN } \\
\text { ScHOOL, COLLEGE, } \\
\text { AND DEPARTMENTAL } \\
\text { (PROFESSIONAL) }\end{array}$} \\
\hline & & $\begin{array}{l}\text { Mini- } \\
\text { mum }\end{array}$ & $\begin{array}{l}\text { Maxi- } \\
\text { mum }\end{array}$ & $\underset{\text { ber }}{\text { Num- }}$ & $\begin{array}{l}\text { Mini- } \\
\text { mum }\end{array}$ & $\begin{array}{c}\text { Maxi- } \\
\text { mum }\end{array}$ & $\underset{\text { ber }}{\text { Num- }}$ & $\begin{array}{l}\text { Mini- } \\
\text { mum }\end{array}$ & $\begin{array}{l}\text { Maxi- } \\
\text { mum }\end{array}$ & $\underset{\text { ber }}{\text { Num- }}$ \\
\hline $\begin{array}{l}\text { 91. Southern Illinois } \ldots \ldots \ldots \ldots \\
\text { 92. Southern Methodist } \ldots \ldots \ldots \ldots \\
\text { 93. Syracuse } \ldots \ldots \ldots \ldots \ldots \ldots \ldots \\
\text { 94. Temple } \ldots \ldots \ldots \ldots \ldots \ldots \ldots \\
\text { 95. Tennessee } \ldots \ldots \ldots \ldots \ldots \ldots \ldots\end{array}$ & $\begin{array}{r}10,080 \\
7,500 \\
11,000 \\
\dddot{9}, 400^{4}\end{array}$ & $\begin{array}{l}7,200 \\
\ddot{4,980} 0 \\
\ddot{6,}, \dot{0} 00\end{array}$ & $\begin{array}{l}7,920 \\
\cdots \cdots \\
\cdots \cdots \\
\cdots \cdots\end{array}$ & $\begin{array}{l}2 \\
i \\
i\end{array}$ & & $\begin{array}{l}6,480 \\
4,500 \\
4,680 \\
5,220 \\
5,200\end{array}$ & $\begin{array}{l}7 \\
6 \\
6 \\
8 \\
6\end{array}$ & $\begin{array}{l}4,560 \\
5,700 \\
3,500 \\
4,360 \\
3,400\end{array}$ & $\begin{array}{l}5,400 \\
6,200 \\
4,200 \\
5,160 \\
6,700\end{array}$ & $\begin{array}{r}2 \\
3 \\
12 \\
5 \\
3\end{array}$ \\
\hline 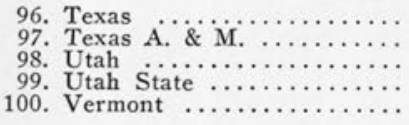 & $\begin{array}{l}10,000 \\
8,700 \\
8,600 \\
5,800^{16} \\
7,200\end{array}$ & $\begin{array}{r}7,400 \\
6,300 \\
6,820 \\
5,100\end{array}$ & $\begin{array}{l}\cdots \cdots \\
\cdots \cdots \\
\cdots \cdots\end{array}$ & \begin{tabular}{r}
1 \\
1 \\
1 \\
\hdashline \\
2
\end{tabular} & $\begin{array}{l}3,840 \\
4,500 \\
3,850 \\
3,650 \\
4,600\end{array}$ & $\begin{array}{l}6,250 \\
4,800 \\
4,300 \\
4,400 \\
4,900\end{array}$ & $\begin{array}{c}10 \\
4 \\
4 \\
6^{16} \\
2\end{array}$ & $\begin{array}{l}3,840 \\
4,000 \\
4,300 \\
3,500\end{array}$ & $\begin{array}{l}5,604 \\
6,320 \\
4,850 \\
\cdots \cdots \\
\cdots \cdots\end{array}$ & $\begin{array}{l}6-2 / 3 \\
2 \\
4 \\
\mathrm{i}\end{array}$ \\
\hline $\begin{array}{l}\text { 101. Virginia }{ }^{35} \\
\text { 102. Washington } \\
\text { 103. Washington }\left(\text { Seattle }{ }^{32}\right) \\
\text { 104. Washington State }\end{array}$ & $\begin{array}{c}9,168 \\
9,500 \\
11,604^{23} \\
10,000 \\
12,900\end{array}$ & $\begin{array}{l}7,032 \\
\cdots \cdots \\
9,250 \\
8,267\end{array}$ & $\begin{array}{l}\cdots \cdots \\
\cdots \cdots \\
\cdots \cdots\end{array}$ & $\begin{array}{l}1 \\
\cdots \\
i \\
1\end{array}$ & $\begin{array}{l}5,640 \\
5,160 \\
5,220^{33} \\
6,260 \\
7,095\end{array}$ & $\begin{array}{l}7,032 \\
5,700 \\
6,600^{33} \\
6,950 \\
8,155\end{array}$ & $\begin{array}{l}5 \\
4 \\
4 \\
4 \\
7\end{array}$ & $\begin{array}{l}4,704 \\
3,250 \\
4,140^{33} \\
7,79 \dot{5}\end{array}$ & $\begin{array}{l}7,344 \\
6,175 \\
5,640^{33} \\
\dddot{7}, 997\end{array}$ & $\begin{array}{r}3 \\
13 \\
14 \\
14 \\
2\end{array}$ \\
\hline 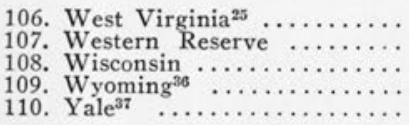 & $\begin{array}{l}7,000 \\
\cdots \cdots{ }^{4} \\
\dddot{9}, 300 \\
\ldots \ldots\end{array}$ & $\begin{array}{l}5,700 \\
9,100^{4} \\
\ldots \ldots\end{array}$ & $\begin{array}{l}\cdots \cdots \\
\dddot{9}, 400 \\
\cdots \cdots \\
\cdots \cdots\end{array}$ & \begin{tabular}{r}
1 \\
1 \\
2 \\
\hdashline \\
3
\end{tabular} & $\begin{array}{l}4,200 \\
4,200 \\
5,200 \\
4,500 \\
\ldots \ldots\end{array}$ & $\begin{array}{l}5,100 \\
5,124 \\
7,124 \\
5,508 \\
\ldots \ldots\end{array}$ & $\begin{array}{l}6 \\
5 \\
8 \\
4 \\
8\end{array}$ & $\begin{array}{r}3,120 \\
3,552 \\
\ldots \ldots\end{array}$ & $\begin{array}{l}\dddot{5}, 120 \\
7,924 \\
\ldots \ldots\end{array}$ & $\begin{array}{r}\ddot{5} \\
10 \\
\ddot{9}\end{array}$ \\
\hline 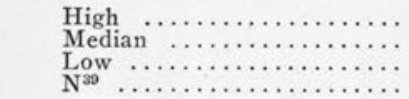 & $\begin{array}{r}15,900 \\
9,200 \\
5,800 \\
74\end{array}$ & $\begin{array}{r}9,384 \\
6,500 \\
3,960 \\
57\end{array}$ & $\begin{array}{r}10,860 \\
8,400 \\
6,000 \\
24\end{array}$ & $\begin{array}{r}8 \\
1 \\
1 \\
74\end{array}$ & $\begin{array}{r}7,095 \\
4,595 \\
3,120 \\
99\end{array}$ & $\begin{array}{r}11,700 \\
5,800 \\
3,800 \\
91\end{array}$ & $\begin{array}{r}43 \\
5 \\
1 \\
103\end{array}$ & $\begin{array}{r}7,795 \\
4,000 \\
2,400 \\
72\end{array}$ & $\begin{array}{r}14,000 \\
6,200 \\
3,960 \\
59\end{array}$ & $\begin{array}{r}30 \\
5 \\
1 \\
76\end{array}$ \\
\hline
\end{tabular}

1. Central library and all agencies. 2. Excludes student assistants and building maintenance staff. 3. Excludes vacations and legal holidays. 4. Not reported or not available. 5. Excludes Schools of Medicine, Pharmacy, and Nursing. 6. Estimate. 7-8. Salary range for the position. 9. Half-time. 10. Excludes additional salary as ViceChancellor. 11. Included with department heads, 12. Excludes full-time positions paid from funds for wages. 13. Excludes librarians on academic department budgets. 14. Excludes nonprofessional library employees on 13. Excludes librarians on academic department budgets. 14. Excludes nonprofessional library employees on academic department budgets. 15. Excludes Denison M. D. Memorial Library, 16. Nine months only. 17. Ex-
cludes Barnard College, Teachers College, New York School of Social Work, and College of Pharmacy. 18. Included with nonprofessional employees. 19. Includes librarians and assistant librarians of New York State Contract Colleges at Cornell. 20. Excludes bindery staff. 21. Excludes T. C. Elementary and High Schools, Hilo Branch, Legislative Reference Bureau, and Industrial Relations. 22. Excludes staffs of Audio-Visual and

\section{College and University Library}

Library ${ }^{1}$

1. Adelphi (N.Y.)

Fiscal
Year
Ending

Student Enrollment

Ending graduate Graduate Extension Stock

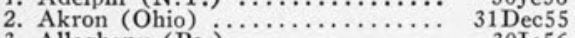

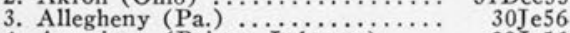

4. American (Beirut, Lebanon)... .3 30 3056

1,773

$2,445^{2}$

1,036

1,063

$\begin{array}{rcr}894 & 1,048 & 79,416 \\ 45 & \ldots . & 102,776 \\ 10 & 18 & 135,912 \\ 77 & \ldots . & 106,000 \\ 10 & \ldots . & 303,303\end{array}$

$\begin{array}{ll}\text { 6. Antioch (Ohio) } & \text { (O.............. } \\ \text { 7. Associated Coll., Claremont (C.alif.) } & 30 \mathrm{Je} 56 \\ 30 \mathrm{Je} 56\end{array}$

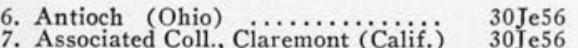

8. Atlanta (Ga.) ................... $31 \mathrm{My} 56$

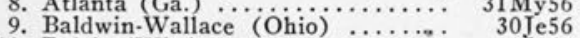

10. Beloit (Wis.) .................... $30 \mathrm{Je56}$

11. Bowdoin (Maine)

$30 \mathrm{Je} 56$

12. Brigham Young (Utah) ....... 31Ag56

14. Bucknell (Pa.) ...............

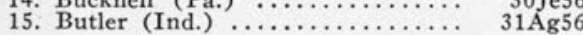

16. Carleton (Minn.)

$30 \mathrm{Je} 5$

17. Central State (Ohio) ............. 30 Ap5

18. Clemson (S.C.) $\ldots \ldots \ldots \ldots \ldots \ldots \ldots \ldots \ldots \ldots$
19. Colby (Maine) $30 \mathrm{Je} 56$
$30 \mathrm{Je} 56$

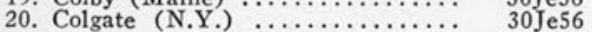

21. College of the Pacific (Calif.) ... 31Ag56

22. Colorado College .............. 30 30

23. Cooper Union (N.Y.) $\ldots \ldots \ldots \ldots \ldots$. 30 Je56

25. Denison (Ohio) $\ldots \ldots \cdots \cdots \cdots \cdots \cdots \cdots \cdots \cdots$

26. DePauw (Ind.) ..............

27. Detroit (Mich.)

28. Dickinson (Pa.) $\ldots \ldots \ldots \ldots \ldots \ldots \ldots$

29. Drake (Iowa)

10. Drexel (Pa.)

$30 \mathrm{Je} 56$

$30 \mathrm{Je} 56$

$30 \mathrm{Je} 56$

$31 \mathrm{My} 56$

1,021

1,616

1,737

987

$3 \dot{8} \dot{4}$

384
578

578

790
7,894

7,894

633

2,007
3,767

903

947

2,917

1,100
1,307

1,239
1,065

1,325

864
1,327

1,957

8,434

871
5,017

3,367

i9

276

20

...

17
649

649
... 303,303

… 88,628

… $\quad 249,755$

$\cdots \quad 142,400$

… 167,500

242,509

i. $\quad 221,889$

ii2 $\quad 240,496$

$\begin{array}{ll}212 & 151,228 \\ \ldots . . & 175,980\end{array}$

... 155,561

$\begin{array}{lr}1 . . & 155,561 \\ \cdots . & 33,549\end{array}$

… 154,782

$\begin{array}{ll}199,394 \\ \cdots . & 217,729\end{array}$

64,878

149

....

64,878
163,003

87,944

… 66,271

.... 127,444

… 180,000

$\begin{array}{ll}0,095 & 100,000 \\ 1,095 & 146,647\end{array}$

$\begin{array}{ll}1,095 & 146,647\end{array}$
Volumes News- PeriAdded papers odicals

$\begin{array}{rrr}5,032 & 10 & 805 \\ 5,509 & 11 & 653 \\ 3,323 & 9 & 402 \\ 5,733 & 24 & 1,400 \\ 6,321 & 13 & 975\end{array}$

5,032

11,006

3,521

2,021

2,417

5,095

9,985

5,817

5,860

3,355

2,049

6,803
5,965

4,056

3,550

2,433

3,246

3,904

13,692

3,111
5,397

6,235 
Salary Statistics, $1955^{-5} 6$ (Group I, Cont.)

\begin{tabular}{|c|c|c|c|c|c|c|c|c|c|c|c|}
\hline All & $\begin{array}{l}\text { TTHER Pr } \\
\text { IL AsSIST }\end{array}$ & $\begin{array}{l}\text { ROFES- } \\
\text { TANTS }\end{array}$ & AlL NoN & $\begin{array}{l}\text { NPROFES } \\
\text { SSISTANT }\end{array}$ & SIONAL & $\begin{array}{r}\text { Tota } \\
\text { IN } \mathrm{F}\end{array}$ & $\begin{array}{l}\text { UMBER OF EM } \\
\text { L-TIME EQUIV }\end{array}$ & $\begin{array}{l}\text { PLOYEES } \\
\text { ALENT }^{2}\end{array}$ & $\begin{array}{l}\text { Working } \\
\text { Hours in }\end{array}$ & $\begin{array}{l}\text { Hours } \\
\text { of Stu- }\end{array}$ & \\
\hline $\begin{array}{l}\text { Mini- } \\
\text { mum }\end{array}$ & $\begin{array}{l}\text { Maxi- } \\
\text { mum }\end{array}$ & $\begin{array}{c}\text { Num- } \\
\text { ber }\end{array}$ & $\begin{array}{l}\text { Mini- } \\
\text { mum }\end{array}$ & $\begin{array}{l}\text { Maxi- } \\
\text { mum }\end{array}$ & $\underset{\text { ber }}{\text { Num- }}$ & $\begin{array}{c}\text { Pro- } \\
\text { fessional }\end{array}$ & $\begin{array}{l}\text { Nonpro- } \\
\text { fessional }\end{array}$ & Total & $\begin{array}{l}\text { Fiscal } \\
\text { Year }^{3}\end{array}$ & $\begin{array}{l}\text { dent As- } \\
\text { sistance }\end{array}$ & \\
\hline $\begin{array}{l}4,200 \\
3,240 \\
3,500 \\
3,800 \\
3,400\end{array}$ & $\begin{array}{l}6,480 \\
4,300 \\
4,200 \\
4,520 \\
5,400\end{array}$ & $\begin{array}{r}9 \\
10 \\
17 \\
18 \\
17\end{array}$ & $\begin{array}{l}2,040 \\
2,000 \\
1,320 \\
2,000 \\
1,800\end{array}$ & $\begin{array}{l}3,360 \\
3,000 \\
2,820 \\
3,840 \\
3,800\end{array}$ & $\begin{array}{l}18 \\
15 \cdot 5 / 6 \\
30 \\
26 \\
41\end{array}$ & $\begin{array}{l}17 \\
18 \\
33 \\
31 \\
28\end{array}$ & $\begin{array}{l}19 \\
15-1 / 3 \\
30-1 / 2 \\
26 \\
38\end{array}$ & $\begin{array}{l}36^{22} \\
33-1 / 3 \\
63-1 / 2 \\
57 \\
66\end{array}$ & $\begin{array}{l}1,739 \\
1,774 \\
1,709 \\
1,611 \\
1,760\end{array}$ & $\begin{array}{l}41,470 \\
22,089 \\
32,103 \\
21,210 \\
16,980\end{array}$ & $\begin{array}{l}91 \\
92 \\
93 \\
94 \\
95\end{array}$ \\
\hline $\begin{array}{l}3,480 \\
3,220 \\
3,600 \\
3,650 \\
3,400\end{array}$ & $\begin{array}{l}5,088 \\
5,300 \\
4,950 \\
3,800 \\
4,000\end{array}$ & $\begin{array}{c}17 \\
12 \\
5 \\
3^{16} \\
8\end{array}$ & $\begin{array}{l}2,280 \\
2,040 \\
2,400 \\
2,150 \\
1,860\end{array}$ & $\begin{array}{l}4,212 \\
3,000 \\
3,320 \\
4,000 \\
3,600\end{array}$ & $\begin{array}{l}55-1 / 4 \\
27 \\
12 \\
8 \\
9\end{array}$ & $\begin{array}{l}33-2 / 3 \\
19^{2} \\
15 \\
13^{4}\end{array}$ & $\begin{array}{r}58 \\
26 \\
13 \\
8 \\
9\end{array}$ & $\begin{array}{l}91-2 / 3 \\
45 \\
28 \\
22^{4}\end{array}$ & $\begin{array}{l}1,864 \\
1,880 \\
1,848 \\
1,480^{16} \\
1,732\end{array}$ & $\begin{array}{r}112,710 \\
15,385 \\
46,000 \\
39,000 \\
8,836\end{array}$ & $\begin{array}{r}98 \\
99 \\
100\end{array}$ \\
\hline $\begin{array}{l}4,128 \\
3,600 \\
3,780^{33} \\
3,925 \\
5,126\end{array}$ & $\begin{array}{l}5,880 \\
5,529 \\
5,640^{33} \\
6,020 \\
7,344\end{array}$ & $\begin{array}{l}18 \\
16 \\
29-3 / 4 \\
14 \\
20^{34}\end{array}$ & $\begin{array}{l}2,112 \\
2,200 \\
2,220^{33} \\
2,520 \\
3,280\end{array}$ & $\begin{array}{l}3,936 \\
2,940 \\
4,680^{33} \\
3,960 \\
5,744\end{array}$ & $\begin{array}{l}42-1 / 2 \\
26-1 / 2 \\
74 \\
34 \\
42^{34}\end{array}$ & $\begin{array}{l}28 \\
30 \\
48-3 / 4 \\
20 \\
32-3 ; 5\end{array}$ & $\begin{array}{l}42-1 / 2 \\
24-1 / 2 \\
74 \\
34 \\
43-1 / 2\end{array}$ & $\begin{array}{c}70-1 / 2 \\
54-1 / 2 \\
122-3 / 4 \\
54 \\
76-1 / 10\end{array}$ & $\begin{array}{l}1,728^{31} \\
1,840 \\
1,824 \\
1,844\end{array}$ & \begin{tabular}{l}
$29,500^{6}$ \\
\hdashline $194^{4}$ \\
32,497 \\
66,314
\end{tabular} & $\begin{array}{l}104 \\
105\end{array}$ \\
\hline $\begin{array}{l}3,750 \\
3,200 \\
3,552 \\
4,116 \\
3,240\end{array}$ & $\begin{array}{l}5,100 \\
4,620 \\
6,626 \\
4,308 \\
4,680\end{array}$ & $\begin{array}{r}9 \\
13 \\
54 \\
4 \\
76\end{array}$ & $\begin{array}{l}2,000 \\
2,400 \\
2,400 \\
2,304 \\
1,740\end{array}$ & $\begin{array}{l}3,600 \\
3,780 \\
3,852 \\
3,000 \\
3,480\end{array}$ & $\begin{array}{r}35 \\
29 \\
32 \\
6 \\
118\end{array}$ & $\begin{array}{r}17 \\
25 \\
74 \\
9 \\
107\end{array}$ & $\begin{array}{c}35 \\
28 \\
32 \\
6 \\
122-47 / 100\end{array}$ & $\begin{array}{l}52 \\
53 \\
106 \\
15 \\
229.47 / 100\end{array}$ & $\begin{array}{l}1,778 \\
1,748 \\
1,872 \\
1,840 \\
1,695\end{array}$ & $\begin{array}{l}31,500 \\
28,000^{\circ} \\
80,123 \\
12,482 \\
44,531\end{array}$ & $\begin{array}{l}109 \\
110\end{array}$ \\
\hline $\begin{array}{r}5,200 \\
3,586 \\
2,700 \\
100\end{array}$ & $\begin{array}{r}10,000 \\
4,800 \\
3,420 \\
98\end{array}$ & $\begin{array}{c}100-3 / 4 \\
14 \\
1 \\
104\end{array}$ & $\begin{array}{r}3,280 \\
2,100 \\
1,320 \\
102\end{array}$ & $\begin{array}{r}6,840 \\
3,540 \\
2,100 \\
104\end{array}$ & $\begin{array}{r}251 \\
27 \\
3 \\
106\end{array}$ & $\begin{array}{r}135 \\
25 \\
2 \\
109\end{array}$ & $\begin{array}{r}251 \\
26 \\
3 \\
110\end{array}$ & $\begin{array}{r}386 \\
52 \\
9 \\
109\end{array}$ & $\begin{array}{r}2,121 \\
1,800 \\
1,400 \\
106\end{array}$ & $\begin{array}{r}186,453 \\
26,176 \\
2,120 \\
106\end{array}$ & $\begin{array}{r}\text { High } \\
\text { Median } \\
\text { Low } \\
\mathrm{N}^{30}\end{array}$ \\
\hline
\end{tabular}

Textbook Rental Departments of Library. 23. Excludes Maryland State College. 24. Excludes Clements, Business Administration, and Lane Hall Libraries. 25. Excludes Schools of Law and Medicine. 26. Excludes Chi cago campus. 27. Excludes Schools of Medicine and Dentistry, 28. Ten months only. 29. Twelve per cent of salary paid for duties as Director of Libraries, Oregon State System of Higher Education. 30. Includes McKissick Library only. 31 . Varies from 1,856 to 1,904 hours depending on length of service. 32. Excludes School of Law and two other autonomous units. 33. Salaries are those being paid as of June 1956. 34. Includes full-time positions only. 35. Excludes branch college libraries. 36. Excludes Law Library. 37. Includes Sterling Memorial five years, 1,880. 39. Number of libraries on which high, median, and low are based.

\section{Statistics, I 955-I956 (Group II)}

\begin{tabular}{|c|c|c|c|c|c|c|c|c|c|c|}
\hline $\begin{array}{c}\text { Staff } \\
\text { Salaries }\end{array}$ & $\begin{array}{l}\text { Student } \\
\text { Service }\end{array}$ & $\begin{array}{l}\text { Total } \\
\text { Staff } \\
\text { Salaries } \\
\text { and } \\
\text { Student } \\
\text { Service }\end{array}$ & $\begin{array}{l}\text { Books } \\
\text { and } \\
\text { Related } \\
\text { Materials }\end{array}$ & Binding & $\begin{array}{l}\text { Total } \\
\text { for } \\
\text { Books } \\
\text { and } \\
\text { Binding }\end{array}$ & $\begin{array}{l}\text { Other } \\
\text { Operat- } \\
\text { ing Ex- } \\
\text { pendi- } \\
\text { tures }\end{array}$ & $\begin{array}{l}\text { Total } \\
\text { Operat- } \\
\text { ing Ex- } \\
\text { pendi- } \\
\text { tures }\end{array}$ & $\begin{array}{l}\text { Per Stu- } \\
\text { dent Op- } \\
\text { erating } \\
\text { Expendi- } \\
\text { tures }\end{array}$ & $\begin{array}{c}\text { Total Edu- } \\
\text { cational and } \\
\text { General In- } \\
\text { stitutional } \\
\text { Expendi- } \\
\text { tures }\end{array}$ & $\begin{array}{c}\text { Ratio of } \\
\text { Library } \\
\text { Expendi- } \\
\text { tures to } \\
\text { Total } \\
\text { (Per Cent }\end{array}$ \\
\hline $\begin{array}{l}37,139 \\
40,861 \\
23,547 \\
56,734 \\
52,531\end{array}$ & $\begin{array}{r}\$ 7,929 \\
14,742 \\
2,668 \\
5,722 \\
5,519\end{array}$ & $\begin{array}{r}\$ 45,068 \\
55,602 \\
26,215 \\
62,456 \\
58,050\end{array}$ & $\begin{array}{r}\$ 16,076 \\
18,020 \\
11,853 \\
36,100 \\
29,434\end{array}$ & $\begin{array}{r}\$ 2,747 \\
2,853 \\
828 \\
2,350 \\
3,431\end{array}$ & $\begin{array}{r}\$ 18,823 \\
20,873 \\
12,681 \\
38,450 \\
32,865\end{array}$ & $\begin{array}{r}\$ 2,693 \\
7,118 \\
1,410 \\
5,095 \\
5,387\end{array}$ & $\begin{array}{r}\$ 66,584 \\
83,593 \\
40,306 \\
106,001 \\
96,302\end{array}$ & $\begin{array}{r}\$ 17.92 \\
33.57 \\
38.53 \\
52.34 \\
89.75\end{array}$ & $\begin{array}{r}\$ 2,556,538 \\
1,669,314 \\
833,915 \\
3,451,234 \\
2,276,180\end{array}$ & $\begin{array}{l}2.60 \\
5.00 \\
4.83 \\
3.07 \\
4.23\end{array}$ \\
\hline $\begin{array}{l}34,550 \\
56,520 \\
35,658 \\
25,245 \\
26,700\end{array}$ & $\begin{array}{r}14,652 \\
14,524 \\
4,842 \\
1,095 \\
3,400\end{array}$ & $\begin{array}{l}49,202 \\
71,044 \\
40,500 \\
26,340 \\
30,100\end{array}$ & $\begin{array}{r}18,925 \\
43,000 \\
9,456 \\
10,119 \\
9,800\end{array}$ & $\begin{array}{l}2,947 \\
7,000 \\
3,049 \\
2,060 \\
1,200\end{array}$ & $\begin{array}{l}21,872 \\
50,000 \\
13,055 \\
12,171 \\
11,000\end{array}$ & $\begin{array}{l}2,019 \\
6,962 \\
4,684 \\
1,136 \\
1,900\end{array}$ & $\begin{array}{r}73,092 \\
128,006 \\
58,239 \\
39,646 \\
43,000\end{array}$ & $\begin{array}{r}71.58 \\
64.50 \\
101.00 \\
22.82 \\
43.00\end{array}$ & $\begin{array}{l}1,213,435 \\
2,967,442 \\
1,070,514^{3} \\
952,261\end{array}$ & $\begin{array}{l}6.07 \\
4.31 \\
3.70^{3} \\
4.51\end{array}$ \\
\hline $\begin{array}{l}27,426 \\
61,950 \\
48,939 \\
45,963 \\
41,617\end{array}$ & $\begin{array}{r}3,960 \\
38,000 \\
2,801 \\
3,138 \\
8,683\end{array}$ & $\begin{array}{l}31,386 \\
99,950 \\
51,740 \\
49,101 \\
50,300\end{array}$ & $\begin{array}{l}14,177 \\
66,500 \\
22,058 \\
21,135 \\
16,245\end{array}$ & $\begin{array}{l}3,464 \\
5,000 \\
4,271 \\
2,972 \\
3,602\end{array}$ & $\begin{array}{l}17,641 \\
71,500 \\
26,329 \\
24,107 \\
19,846\end{array}$ & $\begin{array}{l}2,881 \\
8,050 \\
2,676 \\
2,603 \\
2,906\end{array}$ & $\begin{array}{r}51,908 \\
179,500 \\
80,745 \\
75,811 \\
73,052\end{array}$ & $\begin{array}{r}65.70 \\
21.93 \\
100.00 \\
37.29 \\
19.39\end{array}$ & & $\begin{array}{l}3.80 \\
4.70 \\
5.00 \\
3.82 \\
3.40\end{array}$ \\
\hline $\begin{array}{l}17,410 \\
29,600 \\
59,611 \\
31,953 \\
30,500^{5}\end{array}$ & $\begin{array}{r}5,841 \\
3,000 \\
10,162 \\
3,494 \\
14,309\end{array}$ & $\begin{array}{l}23,251 \\
34,100 \\
69,773 \\
35,447 \\
44,809\end{array}$ & $\begin{array}{r}15,640 \\
8,786 \\
27,447 \\
17,740 \\
16,134\end{array}$ & $\begin{array}{l}1,113 \\
7,299 \\
1,179 \\
1,268\end{array}$ & $\begin{array}{r}16,753 \\
8,786 \\
34,746 \\
18,920 \\
17,402\end{array}$ & $\begin{array}{l}1,590 \\
3,101 \\
3,840 \\
4,281 \\
3,727\end{array}$ & $\begin{array}{r}41,594 \\
45,987 \\
108,359 \\
58,648 \\
65,938\end{array}$ & $\begin{array}{l}46.06 \\
48.70 \\
35.67 \\
53.31 \\
49.00\end{array}$ & $\begin{array}{l}1,229,381 \\
1,128,227 \\
2,637,291 \\
1,113,956 \\
\ldots \ldots \ldots\end{array}$ & $\begin{array}{c}3.40 \\
4.07 \\
4.10 \\
5.30 \\
\ldots\end{array}$ \\
\hline $\begin{array}{l}24,583 \\
24,029 \\
48,060 \\
15,983 \\
30,192^{4}\end{array}$ & $\begin{array}{l}4,690 \\
2,300 \\
9,471 \\
3,307 \\
5,958\end{array}$ & $\begin{array}{l}29,273 \\
26,439 \\
57,531 \\
19,290 \\
36,150\end{array}$ & $\begin{array}{r}13,165 \\
7,958 \\
10,400 \\
12,621 \\
13,846\end{array}$ & $\begin{array}{l}1,577 \\
1,699 \\
3,500 \\
1,206 \\
1,816\end{array}$ & $\begin{array}{r}14,742 \\
9,657 \\
13,900 \\
13,827 \\
15,661\end{array}$ & $\begin{array}{l}1,453 \\
899 \\
3,500 \\
3,870 \\
3,119\end{array}$ & $\begin{array}{l}45,468 \\
36,995 \\
74,981 \\
36,988 \\
54,930\end{array}$ & $\begin{array}{l}30.01 \\
34.00 \\
56.59 \\
42.81 \\
41.38\end{array}$ & $\begin{array}{r}1,125,000 \\
691,200 \\
1,541,767 \\
904,127 \\
1,258,838\end{array}$ & $\begin{array}{l}4.04 \\
5.35 \\
4.86 \\
4.09 \\
4.36\end{array}$ \\
\hline $\begin{array}{l}34,716 \\
67,834 \\
31,766 \\
50,195 \\
44,879^{\circ}\end{array}$ & $\begin{array}{r}3,506 \\
10,891 \\
1,928 \\
13,000 \\
8,066\end{array}$ & $\begin{array}{l}38,322 \\
78,725 \\
33,694 \\
63,195 \\
52,945\end{array}$ & $\begin{array}{l}13,833 \\
50,381 \\
11,024 \\
20,215 \\
18,675\end{array}$ & $\begin{array}{r}1,796 \\
8,116 \\
640 \\
3,200 \\
3,404\end{array}$ & $\begin{array}{l}15,629 \\
58,497 \\
11,664 \\
23,415 \\
22,079\end{array}$ & $\begin{array}{l}6,239 \\
3,078 \\
3,635 \\
2,790\end{array}$ & $\begin{array}{r}60,089 \\
140,298 \\
45,358 \\
90,245 \\
77,814\end{array}$ & & & $\begin{array}{l}3.40 \\
3.60 \\
5.40 \\
4.70 \\
2.41\end{array}$ \\
\hline
\end{tabular}


Library $^{1}$

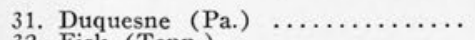

32. Fisk (Tenn.) $\ldots \ldots \ldots \ldots \ldots \ldots$

33. Fort Hayes (Kan )

34. Goucher (Md.).$\ldots \ldots \ldots \ldots \ldots$

36. Hamilton (N.Y.) ...........

37. Haverford (Pa.) $\ldots \ldots \ldots \ldots \ldots \ldots$

38. Holy Cross (Mass.) …........

39. Howard (Ala.) … Howa $\ldots \ldots \ldots \ldots$

41. Idaho State $\ldots \ldots \ldots \ldots \ldots \ldots \ldots$

42. Illinois Inst. of Tech. $\ldots \ldots \ldots$

43. Immaculate Heart (Calif.) .......

45. Kent (Ohio) $\ldots \ldots \ldots \ldots \ldots \ldots \ldots \ldots \ldots \ldots \ldots$

46. Lewis and Clark (Ore.) .......

47. Loyola (Calif.) $\ldots \ldots \ldots \ldots \ldots \ldots \ldots$

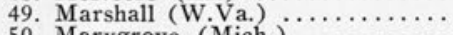

50. Marygrove (Mich.) $\ldots \ldots \ldots \ldots$

51. Michigan Coll. of Mining \& Tech.

52. Middlebury (Vt.) $\ldots \ldots \ldots \ldots \ldots \ldots$

54. Mt. Holyoke (Mass.) $\ldots \ldots \ldots \ldots \ldots$

55. Muhlenberg ( $\mathrm{Pa}.) \quad \ldots \ldots \ldots \ldots \ldots$

56. New Rochelle (N.Y.) . . . . . . .

57. North Texas 58. $\ldots \ldots \ldots \ldots \ldots$

59. Ohio Univ., Athens ............

60. Queens (N.Y.) $\ldots \ldots \ldots \ldots \ldots \ldots$

61. Redlands (Calif.) ...........

62. Reed (Ore.) $\ldots \ldots \ldots \ldots \ldots \ldots \ldots \ldots$

64. Roosevelt (Iil.) $\ldots \ldots \ldots \ldots \ldots \ldots$

66. St. John's (Minn.)

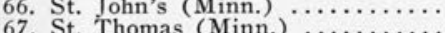

68. San Francisco (Calif.) $\ldots \ldots \ldots$

70. Santa Clara (Calif.) $\ldots \ldots \ldots \ldots$

71. Skidmore (N.Y.) $\ldots \ldots \ldots \ldots \ldots$

72. Smith (Mass.)

73. South Dakota State

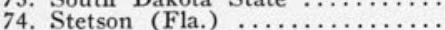

75. Swarthmore ( $\mathrm{Pa}$. $\ldots \ldots \ldots \ldots$

76. Sweet Briar (Va.) ............

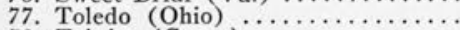

78. Trinity (Conn.) $\ldots \ldots \ldots \ldots \ldots \ldots$

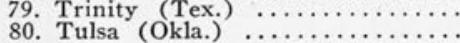

81. Valparaiso (Ind.)

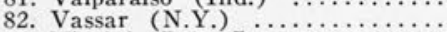

83. Virginia Poly. Inst. $\ldots \ldots \ldots \ldots \ldots$

84. Wake Forest (N.C.) … . . .

85. Wellesley (Mass.) ............

86. Wesleyan (Conn.)

(I11.)

88. Wheaton (Mass.) $\ldots \ldots \ldots \ldots \ldots \ldots$

89. Wichita (Kan.)

90. William and Mary $\left(\hat{V}_{\mathrm{a}}.\right) \ldots \ldots$.

91. Williams (Mass.)

92. Wooster (Ohio) $\ldots \ldots \ldots \ldots \ldots$

Median $\ldots \ldots \ldots \ldots \ldots \ldots \ldots \ldots \ldots \ldots \ldots \ldots$

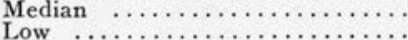

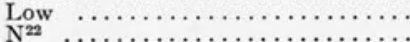

Fiscal
Year
Ending

Student EnRollment

$30 \mathrm{Je} 56$

$31\}$ y 56

$30 \mathrm{Je} 56$

$30 \mathrm{Je} 56$

$30 \mathrm{Je} 56$

$31 \mathrm{Ag} 56$

$30 \mathrm{Je} 56$

$31 \mathrm{Ag} 56$

$30 \mathrm{Je} 56$

31 Âg 56

1 Jy 56

$30 \mathrm{Je} 56$

$31 \mathrm{My} 56$

31 Jy 56

$30 \mathrm{Je} 56$

$30 \mathrm{Je} 56$

$30 \mathrm{Je} 56$

30 e 56
$30 \mathrm{Je} 56$

$30 \mathrm{Je} 56$

31 Ảg 56

$30 \mathrm{Je} 56$

$31 \mathrm{Ag} 56$

$30 \mathrm{Je} 56$

$30 \mathrm{Je} 56$

$30 \mathrm{Je} 56$

30 Je56

$30 \mathrm{Je} 56$

$31 \mathrm{Ag} 56$
$30 \mathrm{Je} 56$

$30 \mathrm{Je} 56$

31 My 56

$30 \mathrm{Je} 56$

$30 \mathrm{Je} 56$

$30 \mathrm{Je} 56$

$30 \mathrm{Je} 56$
$30 \mathrm{Je} 56$

31 Âg 56

30 Je56

$30 \mathrm{Je} 56$

$30 \mathrm{Je} 56$

$30 \mathrm{e} 56$
31 My 56

31 My 56
31 My 56

$30 \mathrm{Je} 56$

$30 \mathrm{Je} 56$

$30 \mathrm{Je} 56$

$30 \mathrm{Je} 56$

$30 \mathrm{Je} 56$

31 A g 56

$30 \mathrm{Je} 56$

$30 \mathrm{Je} 56$
$30 \mathrm{Je} 56$

$30 \mathrm{Je} 56$

$30 \mathrm{Ag} 56$

1. Central library and all agencies. 2. Does not not available. 4. Salaries for 11 months only. 5. Professional only. 6 . Includes only part of Director's salary.
7. Total enrollment 1,765 . 7a. Total enrollment 883 . 8. Includes $\$ 7,000$ non-salaried services. 9. Includes $\$ 20,000$

Total

graduate Graduate Extension Stock

3,584
695
1,957
634
843
633
459
1,823
1,440
3,380

\begin{tabular}{|c|c|c|}
\hline 339 & . & 70,8 \\
\hline 41 & $\ldots$ & 126,2 \\
\hline 125 & 484 & \\
\hline 15 & $\ldots$ & 97, \\
\hline$\cdots$ & $\ldots$ & 121 \\
\hline$\cdots$ & $\ldots$ & 241 \\
\hline & $\cdots$ & 204 \\
\hline & $\ddot{2}, 02 \dot{8}$ & 70 \\
\hline 235 & 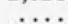 & 3 \\
\hline
\end{tabular}

Volumes News- PeriAdded papers odicals

$\begin{array}{rrr}5,955 & 7 & 696 \\ 3,613 & 47 & 523 \\ 6,500 & 78 & 533 \\ 2,207 & 3 & 473 \\ 2,599 & 6 & 539 \\ & & \\ 5,335 & 8 & 550 \\ 4,180 & 6 & 640 \\ 6,511 & 29 & 370 \\ 2,813 & 22 & 661 \\ 12,037 & 41 & 1,762\end{array}$

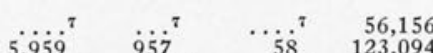

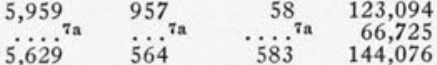

$\begin{array}{rrrr}5,629 & 564 & 583 & 144,076 \\ 793 & \ldots & \ldots & 86,021\end{array}$

$5,820 \quad 81 \quad 937$

$\begin{array}{lll}2,126 & \text { ii } & 950 \\ 6,648 & \text { 17 } & 493\end{array}$

$\begin{array}{lll}7,806 & 10 & 815\end{array}$

1,024
1,166

1,166

2,980

699

32
398

$\dot{3} \dot{6} \dot{1}$

... 44,632

... 86,341

\begin{tabular}{lr}
$33 \dot{3}$ & 23,000 \\
\hdashline & 102,471
\end{tabular}

2,242

1,258

472
1,204

1,204
828

...

829

5,053

1,251

3,924

1,033

1,033
632

608
3,503

942

1,000

1,333

2,860

1,097
389

25

10

68
43
$\cdots$

... 74,157

3,299
3,341

4,000

3,535

3,340

70,076

... 126,838

$\begin{array}{ll}\cdots & 121,954 \\ \cdots & \end{array}$

1,834

3,333

3,218

5,758
2,750

246,634
91,818

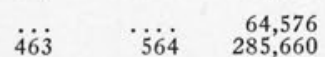

1,958

$\begin{array}{rrr}463 & 564 & 285,660 \\ 185 & \because 4 \dot{8} & 121,632\end{array}$

$750 \quad 448 \quad 254,795$

17,003

10,918

13,312

$\begin{array}{llr}65 & \cdots & 99,882\end{array}$

5,578

4,391

$\begin{array}{rrr} & 320 & 94,540 \\ 261 & \ldots & 100,936\end{array}$

4,657
7,000

7,000
2,273

85,421

4,126

2,110
5,469

5,469

6,382

1,054

2,243

2,605
1,536

1,536
907

$\begin{array}{lll}75 & \cdots & 99,862\end{array}$

$\begin{array}{rlr}50 & \cdots & 55,258 \\ 180 & \cdots & 60,755\end{array}$

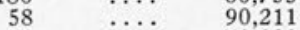

69,000

4,199

$\because 60 \quad \cdots \cdots \quad 80,135$

$\begin{array}{lll}60 & \cdots & 406,929\end{array}$

$\begin{array}{lll}79 & \ldots & 112,739\end{array}$

$\begin{array}{llr}84 & \cdots & 91,069 \\ \cdots & \cdots & 200,434\end{array}$

9,213

4,262

3,272
5,610

... 85,993

480
5,170

5,170

906
1,480

320

274
275

2,107

12,637

... 363,936

363,936
72,885

72,885
164,847

6,150

5,808
8,690

2,350

1,428

3,754

1,750

$\begin{array}{lll}\cdots & 264 \quad 82,500\end{array}$

$\begin{array}{rlr}6 & \cdots & 309,662 \\ 230 & \cdots & 202,100\end{array}$

4,279

6,449

8,740
5,532

5,718

$\begin{array}{rrrr}774 & 62 & 151 & 418,269\end{array}$

11,383

774
1,697
546

546
4,087
$\ldots .21$

2,455

8,614
7,378

4,138

3,164

17,003

4,524
248
92

$\begin{array}{rrrr}8,434 & 957 & 3,002 & 418,269 \\ 1,316 & 76 & 448 & 121,552 \\ 389 & 1 & 18 & 23,000 \\ 88 & 64 & 17 & 92\end{array}$

$24 \quad 485$

$10 \quad 337$

21
11

$20 \quad 593$

$27 \quad 580$

$13 \quad 480$

$14 \quad 717^{11}$

$16 \quad 39$

$\begin{array}{rr}33 & 1,689 \\ 14 & 707\end{array}$

$17 \quad 1,138$

$9 \quad 771$

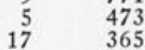

$22 \quad 646$

$25 \quad 725$

$10 \quad 445$

$1 \quad 845$

$9 \quad 534$

$\begin{array}{rr}30 & 1,450 \\ 21 & 950\end{array}$

$13 \quad 662$

1,029

$10 \quad 453$

4
18

$\begin{array}{ll}18 & 551 \\ 12 & 418\end{array}$

$\begin{array}{rr}14 & 524 \\ 28 & 1,381\end{array}$

$\begin{array}{ll}97 & 2,031 \\ 31 & 1,171\end{array}$

$21 \quad 1,145$

121,200

$\begin{array}{rr}8 & 574 \\ 9 & 375\end{array}$

$\begin{array}{lr}10 & 846 \\ 18 & 1,287\end{array}$

9
9 400

$97 \quad 2,293$

$\begin{array}{rr}97 & 2,293 \\ 14 & 636 \\ 1 & 320 \\ 91 & 92\end{array}$ non-salaried services. 10. Includes $\$ 6,500$ non-salaried services. 11. Plus 285 received through Hampshire Inter- 
Statistics, $1955^{-5} 6$ (Group II, Cont.)

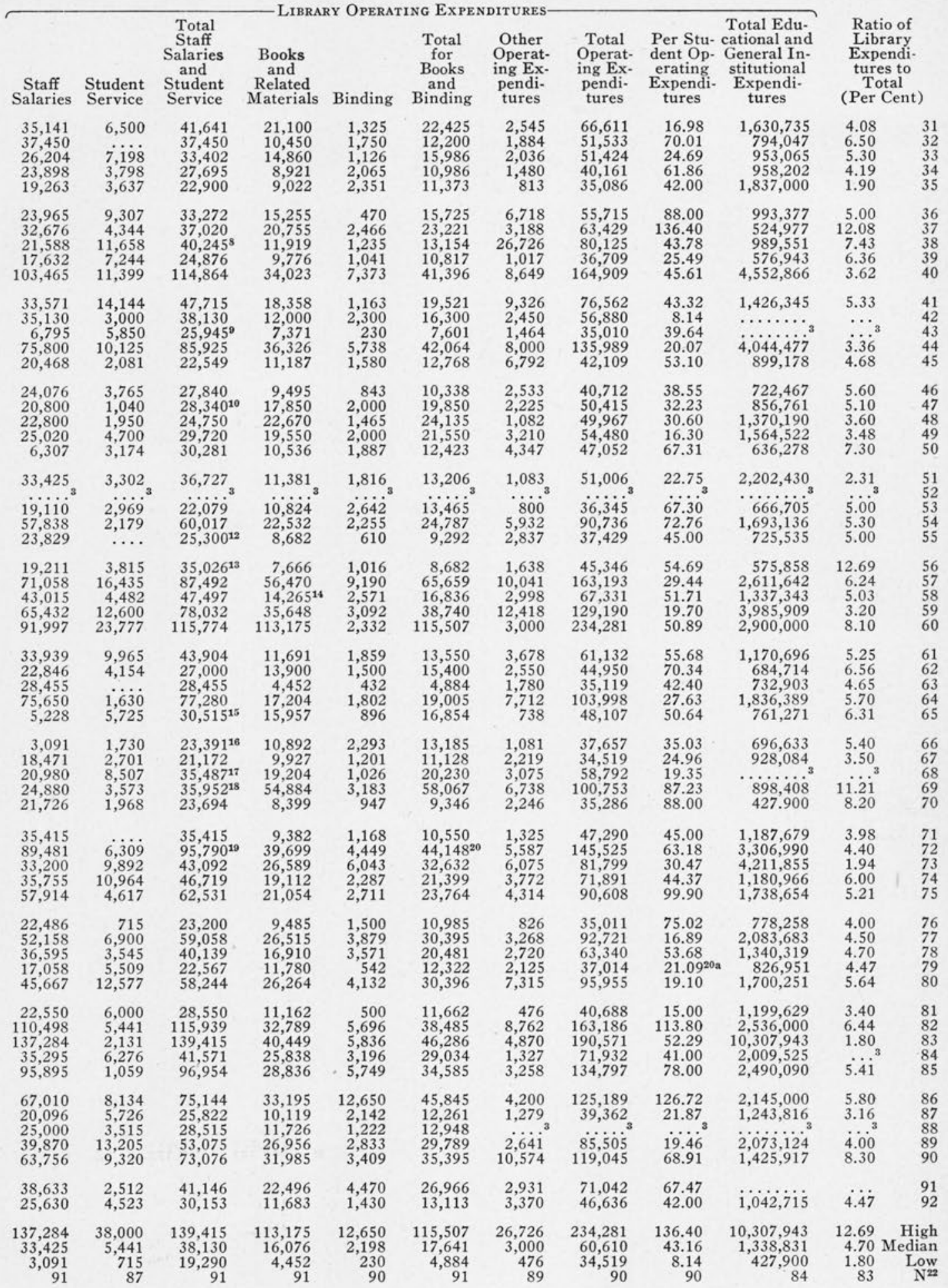

additional spent from gifts not in budget. 15. Includes $\$ 19,562$ non-salaried services. 16. Includes $\$ 19,570$ nonsalaried services. 17. Includes $\$ 6,000$ non-salaried services. 18. Includes $\$ 7,500$ non-salaried services. 19. Does not include music and art libraries staff salaries. 20. Includes funds for music and art libraries. 20. $\$$ Richmond. 22. Number of libraries on which high, median and low are based. 


\section{College and University Library Salary}

Library $^{3}$

1. Adelphi (N.Y.)

2. Akron (Ohio)

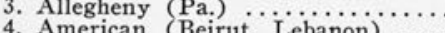

5. Amherst (Mass.)

6. Antioch (Ohio)

7. Associated Coll., Claremont (Calif.) 8. Atlanta (Ga.)

9. Baldwin-Wallace (Öhio) ............

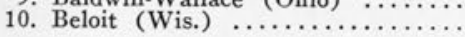

11. Bowdoin (Maine)

12. Brigham Young (Utah) $\ldots \ldots \ldots$

13. Bryn Mawr (Pa.) ...............

14. Bucknell (Pa.) ..................

15. Butler (In $(\mathrm{Pa})$

16. Carleton (Minn.)

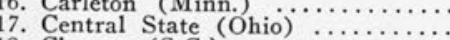

18. Clemson (S.C.)

19. Colby (Maine)

1. College of the Pacific (Calif) .....

22. Colorado State

23. Cooper Union (N.Y.) $\ldots \ldots \ldots \ldots . .$.

24. Davidson (N.C.) …...........

25. Denison (Ohio)

26. DePauw (Ind.)

28. Dickinson ( $\mathrm{Pa}$.

29. Drake (Iowa)

30. Drexel (Pa.)

31. Duquesne (Pa.)

32. Fisk (Tenn.)

33. Fort Hayes (Kan.)

34. Goucher (Md.)

36. Hamilton (N.Y.)

37. Haverford ( $\mathrm{Pa}$. )

39. Howard (Ala.)

41. Idaho State

42. Illinois Inst $\ldots \ldots \ldots$

43. Immacula

43. Immaculate Heart (Calif.) $\ldots \ldots \ldots$

46. Lewis and Clark (Ore.) ........

47. Loyola (Calif.) $\ldots \ldots \ldots \ldots \ldots \ldots$

48. McNeese (La.) $\ldots \ldots \ldots \ldots \ldots \ldots$

50. Mar

51. Michigan Coll. of Mining \& Tech. 52. Middlebury (Vt.) $\ldots \ldots \ldots \ldots \ldots$

53. Mills (Calif.) (M...

5. Muhlenberg ( $\left.\mathrm{Pa}_{3}\right)$

56. New Rochelle (N.Y.)

57. North Texas

58. Occidental (Calif.) $\ldots \ldots \ldots \ldots \ldots \ldots$

59. Ohio Univ., Athens

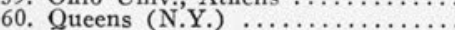

61. Redlands (Calif.)

62. Reed (Ore.)

63. Roosevelt (Iil.)

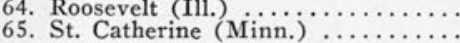

\begin{tabular}{|c|c|c|c|}
\hline \multirow[b]{2}{*}{$\underset{\text { brarian }}{\mathrm{Li}-}$} & \multicolumn{3}{|c|}{$\begin{array}{c}\text { AsSOCIATE OR } \\
\text { ASSISTANT } \\
\text { CHIEF LIBRARIAN }\end{array}$} \\
\hline & $\begin{array}{l}\text { Mini- } \\
\text { mum }\end{array}$ & Maxi- & $\begin{array}{c}\text { Num. } \\
\text { ber }\end{array}$ \\
\hline$\$ \ldots W_{2}^{2}$ & $\$ \ldots$ & $\$ \ldots$. & $\cdots_{2}$ \\
\hline$\ldots^{2}{ }^{2}$ & $\ldots{ }^{2}$ & $\ldots{ }^{2}$ & $\ldots^{2}$ \\
\hline$\ldots \cdots^{2}$ & $\ldots^{2}$ & $\cdots_{2}^{2}$ & $i^{2}$ \\
\hline 6,750 & $\ldots$ & 5,500 & 1 \\
\hline $7,500_{2}$ & $\cdots v_{2}$ & $\cdots$ & $\therefore$ \\
\hline$\ddot{6}, \overline{70} \dot{0}^{2}$ & $\ldots{ }^{2}$ & $\ldots{ }^{2}$ &.$^{2}$ \\
\hline 6,300 & $\ldots$ & $\ldots$. & .. \\
\hline $\begin{array}{l}8,000 \\
6,700\end{array}$ & $\begin{array}{l}5,400 \\
5,000\end{array}$ & 5,100 & $\begin{array}{l}1 \\
2\end{array}$ \\
\hline $6.500^{2}$ & $\cdots$ & $\ldots$ & 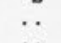 \\
\hline 6,000 & 3,350 & ..... & i \\
\hline$\because \ddot{0}^{2}$ & $\ldots^{2}$ & $\ldots^{2}$ &.$^{2}$ \\
\hline 7,200 & 4,860 & $\cdots$ & i \\
\hline 7,500 & 4,500 & $\cdots$ & 1 \\
\hline & & $\cdots$ & \\
\hline $\begin{array}{l}1,750^{7} \\
5,000\end{array}$ & 4.400 & $\cdots$ & ${ }^{i} \mathrm{i}$ \\
\hline$\because 60^{2}$ & 5,400 & $\cdots$ & 1 \\
\hline 6,600 & $\cdots$ & $\cdots$ & . \\
\hline & .... & $\cdots$ & $\cdot$ \\
\hline$\ldots \ldots^{2}$ & $\ldots^{2}$ & $\ldots^{2}$ & 1 \\
\hline$\ddot{6}, \overline{5} 00^{2}$ & $\cdots$ & $\ldots$. & $\because$ \\
\hline$\ldots m^{2}$ & $\ldots .^{2}$ & 5,300 & $\ddot{i}$ \\
\hline 5,500 & $\cdots_{2}$ & $\cdots$ & $\cdots$ \\
\hline$\dddot{6}, 2 i 0^{2}$ & $\ldots$ & $\ldots^{2}$ &.$^{2}$ \\
\hline$\dddot{5}, 250^{2}$ & $\cdots^{2}$ & $\cdots^{2}$ & $\cdots^{2}$ \\
\hline & & $\cdots$ & $\cdots$ \\
\hline$\ddot{2} 2 \ddot{0}^{2}$ & $\ddot{3} 60^{2}$ & $39 \dot{0}^{2}$ & 1 \\
\hline 7,000 & $\ldots$ & 5,000 & 1 \\
\hline $\begin{array}{l}4,200 \\
7,785\end{array}$ & $\ldots$ & $\ldots$ & .. \\
\hline & $\cdots$ & $\cdots$ & . \\
\hline $\begin{array}{l}6,000 \\
8,300\end{array}$ & $\ldots$. & $\ldots$. & 2 \\
\hline & $\ldots{ }^{2}$ & $\cdots{ }^{\cdots}{ }_{2}$ & i \\
\hline 9,100 & 5,000 & 6,000 & 1 \\
\hline & $\cdots$ & $\cdots$ & 1 \\
\hline 6,500 & .... & $5,000^{15}$ & $5 \quad 1$ \\
\hline 6,900 & $5,400^{14}$ & $\cdots$ & $\mathrm{i}$ \\
\hline $\begin{array}{l}5,720 \\
5,500\end{array}$ & $\cdots$ & $4, \overline{5} 000$ & i \\
\hline 6,350 & 4,420 & 4,900 & 2 \\
\hline 5,300 & $\cdots \cdots$ & $\ldots$. & \\
\hline$\ddot{6,5000^{2}}$ & $4,700^{2}$ & $\ldots$ & $\begin{array}{l}1 \\
1\end{array}$ \\
\hline & & & \\
\hline 7,744 & 5,200 & $\ldots$ & 1 \\
\hline $\begin{array}{l}6,000 \\
8,823\end{array}$ & $\cdots$ & $\ldots$ & \\
\hline 8,650 & 6,450 & 8,050 & $i$ \\
\hline 6,000 & $\ldots$ & $\cdots$ & . \\
\hline & ... & $\ldots$ & \\
\hline 6,000 & .... & .... & $\therefore$ \\
\hline & $\ldots$ & $\ldots$ & 1 \\
\hline
\end{tabular}

Department Heads

$\underset{\text { mumi- }}{\text { Maxi- Num }}$

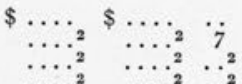

$\begin{array}{lll}\cdots \cdots_{2}^{2} & \cdots \cdots_{2}^{2} & \cdots\end{array}$

$4,300 \quad 4,950 \quad 3$

$\begin{array}{lll}4,800 & 4,000 & 3 \\ 3,600 & 4,300 & 3\end{array}$

$3,600 \quad 4,300 \quad 3$

$4,000 \quad 4,400 \quad 3$

$3,800 \quad 4,000$

$4,400 \quad 5,000$

$4,400-5,000$

$\begin{array}{ll}4,100 & 4,900 \\ 3,850 & 4,500\end{array}$

3,204

$3, \dddot{3}^{2} \dot{0}^{2} \quad 3, m^{2} \dot{0}^{2}$

$4,404 \quad 5,604 \quad 4$

$\begin{array}{lll}3,500 & 4,500 \\ 4,200 & 4,700\end{array}$

$4,500 \quad 5,000 \quad 4$

$\begin{array}{lll}3,400 & & \\ 4,800 & 5,000 & 2\end{array}$

$\begin{array}{ll}3,650^{8} & 3,800^{8} \\ 4,300 & \ldots\end{array}$

$4,400^{2} \quad \cdots^{2}, \dot{0}^{2} \quad 3$

$\begin{array}{lll}\cdots_{2} \quad \cdots_{2} & \cdots\end{array}$

$4,100 \quad 4,700$

$\begin{array}{lll}3,600 & 4,400 & 3 \\ 2 & & 3\end{array}$

$4,23 \dot{5}^{2} \quad \dddot{5}_{3}, 720^{2} \ddot{3}^{2}$

$3,900 \quad 4,100{ }^{2} \ddot{4}^{2}$

$3,084^{2} \quad 3,600^{2} \quad 2$

$3,400 \quad 4,500 \quad 2$

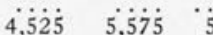

$3,900 \quad 4,200 \quad 2$

$\begin{array}{ll}3,900 & 4,200 \\ 3,600 & 5,300\end{array}$

$\begin{array}{ll}3,800^{2} & 5,500^{2} \\ 3,500 & 4,700\end{array}$

……

3,870

…

3,870
3,700

4,625

$\dddot{3}^{2} \quad \dddot{\cdots}^{2}{ }^{2} \quad \ddot{3}^{2}$

$3,700^{2} \quad \ldots \cdots^{2} \quad{ }^{2}$

$4,000 \quad 5,000 \quad \because$

$3,500 \quad 5,000$

$\begin{aligned} & 4,600 \\ & 5,500\end{aligned} \quad 5,046$

6,750

4,000
3,500

3,000

4,350
3,531

4,400
4,100

5,000

4,000
Head Librarian

School, College,
and Depart Mental

(PROFESSIONAL)

Mini- Maxi- Num-

mum mum be

$\$ \ldots . \$ \ldots .$.

$\ldots^{2} \quad \ldots 2^{2} \cdots^{2}$

$\cdots+{ }^{2}$

$\begin{array}{lll}\cdots \cdots^{2} & \ldots{ }^{2} & \cdots\end{array}$

$\cdots \cdots$

$\ddot{4,400} \quad \ddot{4,400} \quad \ddot{2}$

... ....

$\cdots \cdots, \quad \cdots$

$\ldots^{2} \quad \ldots{ }^{2} \ldots^{2}$

$\cdots$

$\therefore$

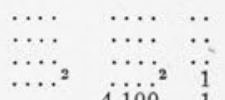

$\cdots_{2} \quad \cdots_{2} \quad \cdots_{2}$

... $\ldots . .$.

$\begin{array}{lll}\cdots & \cdots & \cdots\end{array}$

$4,660 \quad 5,500 \quad 3$

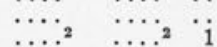

$\begin{array}{lll}\cdots & \cdots & \cdots\end{array}$

$5,000 \quad \cdots \cdots$

$4,500^{14} \quad \cdots \cdots \cdot$ i

$\ddot{3,000} \quad \ldots .$.

$\begin{array}{lll}\cdots & \cdots & \cdots\end{array}$

$\begin{array}{lll}\cdots & \cdots & \cdots\end{array}$

$\begin{array}{lll}\cdots & \cdots & \cdots\end{array}$

$5,200 \quad \ldots . \cdots \quad{ }_{1}$

$\ddot{4,100 \quad 6,200} \quad \ddot{5}$

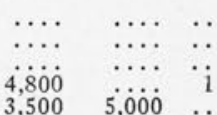


Statistics, ' I 955-I 956 (Group II)

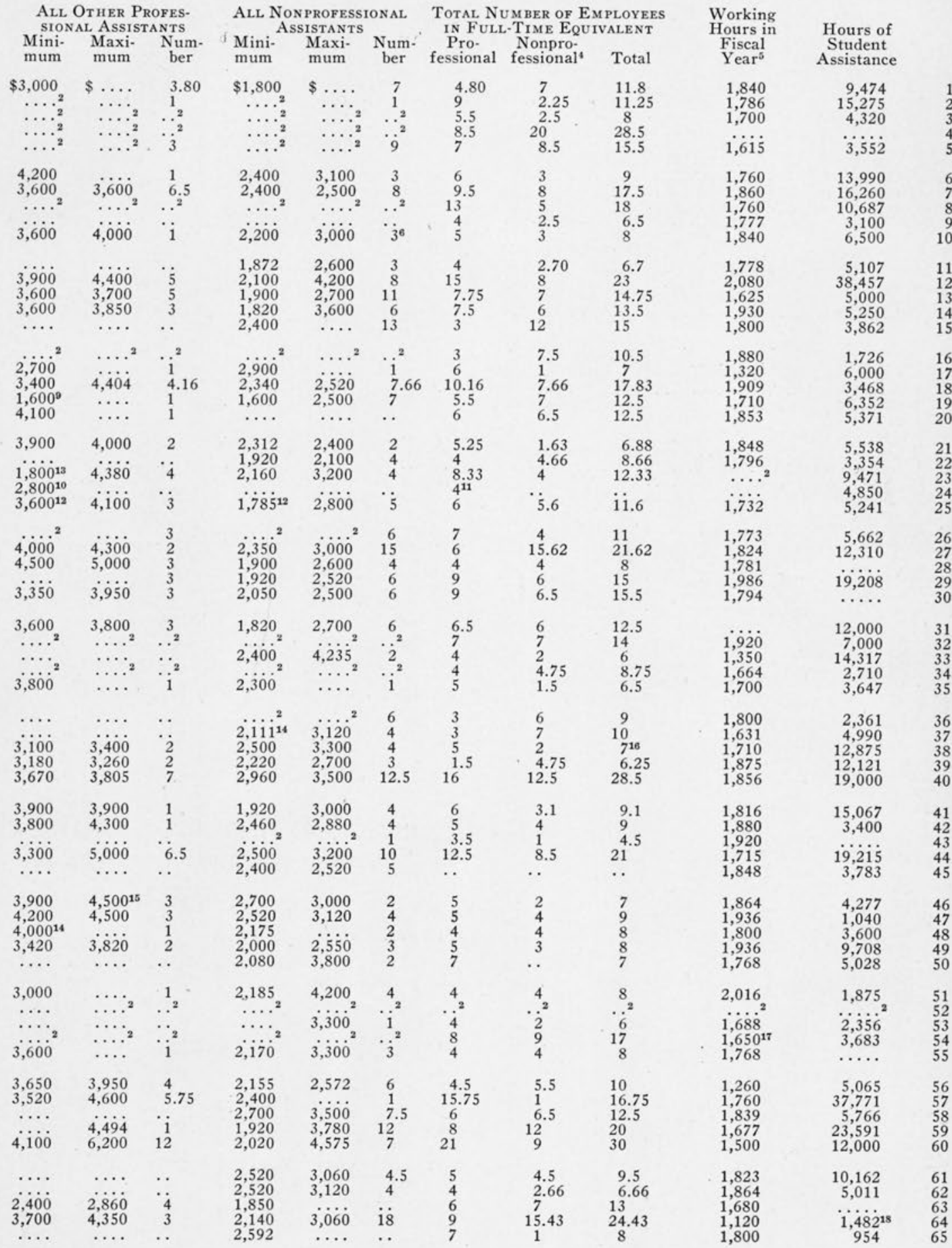


Library $^{3}$

66. St. John's (Minn.)

67. St. Thomas (Minn ) .............

68. San Francisco (Cai

69. Santa Clara (Calif.)

70. Sarah Lawrence (N.Y.) ............

71. Skidmore (N.Y.)

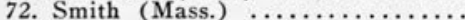

73. South Dakota State

74. Stetson (Fla.

75. Swarthmore (Pa.) $\ldots \ldots \ldots \ldots \ldots \ldots$

76. Sweet Briar (Va.)

77. Toledo (Ohio)

78. Trinity (Conn.)

79. Trinity (Tex.)

80. Tulsa (Okla.)

81. Valparaiso (Ind.)

82. Vassar (N.Y.)

83. Virginia Poly. Inst.

85. Wellesley (Mass.) ............

86. Wesleyan (Conn.) .............

87. Wheaton (IIl.)

88. Wheaton (Mass.) $\ldots \ldots \ldots \ldots \ldots \ldots \ldots . . .6$.

89. Wichita (Kan.)

90. William and Mary (Va.)

91. Williams (Mass.)

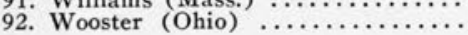

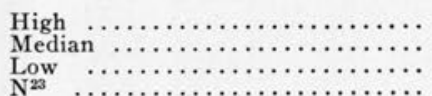

\begin{tabular}{|c|c|c|c|}
\hline \multirow[b]{2}{*}{$\underset{\text { brarian }}{\mathrm{Li}-}$} & \multicolumn{3}{|c|}{$\begin{array}{c}\text { Assistant } \\
\text { CHIEF LIBRARIAN }\end{array}$} \\
\hline & $\begin{array}{l}\text { Mini- } \\
\text { mum }\end{array}$ & $\begin{array}{l}\text { Maxi- } \\
\text { mum }\end{array}$ & $\begin{array}{l}\text { Num. } \\
\text { ber }\end{array}$ \\
\hline & 7,000 & ... & 1 \\
\hline & ..... & 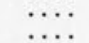 & $\because$ \\
\hline & & $\cdots$ & \\
\hline & 4,800 & $\cdots$ & 1 \\
\hline $5,450_{2}$ & $4,500_{2}$ & 5,250 & \\
\hline 7,000 & & & \\
\hline & 5,500 & $\ldots$ & 1 \\
\hline 5,300 & 4,000 & & \\
\hline & $\cdots_{2}^{2}$ & $\cdots_{2}^{2}$ & \\
\hline & $\ldots$. & $\ldots$ & 1 \\
\hline & $\cdots$ & $\ldots$ & \\
\hline & ... & $\cdots$ & .. \\
\hline & 5,160 & $6,43 \dot{3}$ & 2 \\
\hline & $\ddot{5,800}$ & $5, \ddot{8} 0 \dot{0}$ & 4 \\
\hline 10,30 & & $\cdots$ & .. \\
\hline & $4,500_{2}$ & $\ldots^{2}$ & $\because{ }^{2}$ \\
\hline $\begin{array}{l}6,500 \\
7,032\end{array}$ & ... & $\cdots$ & \\
\hline & 580 & & \\
\hline & $\ldots, \ldots^{2}$ & $\cdots^{2}{ }^{2}$ & \\
\hline 10 & 7,000 & & \\
\hline $\begin{array}{l}6,5 \\
3,9\end{array}$ & $\begin{array}{l}5,400 \\
3,350\end{array}$ & 3,990 & \\
\hline & 23 & 11 & 37 \\
\hline
\end{tabular}

Department Heads

Mini- Maxi- Num

mum mum ber

$3,600 \quad \ldots \ldots{ }_{1.75}$

...

$\ldots$

$\cdots \cdots$

$\begin{array}{lll}3,750 & 4,800 & \ddot{6} \\ 4,000 & 4,600 & 4 \\ 4,000 & 4,700 & 3 \\ \ldots 2^{2} & \ldots 2^{2} & 4\end{array}$

Scho Librarian

AND DEPARTMENTAL

(PRofessional)

Mini- Maxi- Num

mum mum ber

3,700

$2,400^{2}$

$\cdots 2^{2} \quad{ }^{1}$

$4,200-4,760$

$\begin{array}{lll}5,600 & 6,1000 & \ddot{4} \\ 4,320 & 5,400 & 4 \\ 3,600 & 3,800 & 4\end{array}$

$\begin{array}{lll}3,250 & 3000 & \ddot{3} \\ \ddot{4,000} & \ddot{5}, 0 \ddot{0}^{2} & \ddot{4}^{2} \\ 4,320 & 5,400 & 5\end{array}$

$4,700,4,800,2$

5,600
3,870

2,400

$\begin{array}{rr}6,750 & 7 \\ 4,700 & 3 \\ 3,600 & 1 \\ 49 & 64\end{array}$

1. Salaries are as of September 1, 1956. 2. Not reported or not available. 3. Central library and all agencies. 4. Excludes student assistants and building maintenance staff. 5. Excludes vacations and legal holidays. 6. Includes two nonprofessionals working half time. 7. 25 per cent appointment. 8. Actual salaries. 9. Half-time. 10. Part-time, actual salary. 11. One position part time. 12. Ten months. 13. One-third time. 14. Nine months. 15.

\section{College and University Library}

Library $^{1}$

1. Agnes Scott (Ga.)

Alabama College

3. Alaska ......................

4. Anderson (Ind.)

5. Arkansas A. \& M. .............

6. Arkansas State

7. Ashland (Ohio)

8. Augustana (S.D.)

9. Aurora (IIl.)

(n)

11. Bates (Maine)

12. Beaver (Pa.)

13. Belhaven (Miss.)

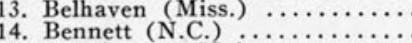

15. Bennington (Vt.) $\ldots \ldots \ldots \ldots \ldots$

16. Bethany (Kan.)

17. Birmingham-Southern (Ala.)

18. Catawba (N.C.)

19. Cedar Crest (Pa) $\ldots \ldots \ldots \ldots$

20. Chapman (Calif)

21. Coe (Iowa)

1. Coe (lowa)

23. Coll of the $\ldots \ldots \ldots \ldots$

3. Coll of the Ozarks (Ark. $\cdots$

25. Concord (W.Va.) $\ldots \ldots \ldots \ldots$.
Fiscal
Year
Ending

$30 \mathrm{Je} 56$

$31 \mathrm{Ag} 56$

$30 \mathrm{Je} 56$

$30 \mathrm{Je} 5$

$30 \mathrm{Je} 56$

$30 \mathrm{Je} 56$

$10 \mathrm{Je} 56$

30 บe56

$30 \mathrm{Ye} 56$

$30 \mathrm{e} 56$

$30 \mathrm{Je} 56$

$31 \mathrm{My} 56$

$15 \mathrm{Je} 56$

$30 \mathrm{Je} 56$

$31 \mathrm{My} 56$

$31 \mathrm{Ag} 56$

$30 \mathrm{Je} 56$

31 Ag 56

$31 \mathrm{Ag} 56$

$31 \mathrm{Jy} 56$

$30 \mathrm{y} 56$

$30 \mathrm{Je} 56$

\section{Student Enrollment \\ Total
Under- Total Total graduate Graduate Extension}

Book
Stock

Volumes

News- Peri-

562
485
429

429

998

$\ddot{2}$

i4i5

$65,523^{4}$

$67,613^{4}$

39,499

$29,631^{4}$

2,202

652
1,111

1,111

212

832

564

177

424
337

291

976
629

629
427

359

824

824
246

1,004

1,004 $\dddot{46}$

47,192

29,000

38,474

39,632
69,584

$99,938^{4}$

$34,000^{4}$

24,088

33,045

33,832

32,000

81,527

$46,415^{4}$

29,947

63,732

29,542

$40,025^{4}$
2,250

1,650

1,958

1,050
420

2,548

1,300
1,835

1,041

2,425

2,916

1,533

1,092
1,379

944

750
1,832

1,832
1,679

919
2,282

180
408
2,237
2,135
714 ers odicals

6
17

$16 \quad 513$

5
18

$11 \quad 470$

$6 \quad 290$

$10 \quad 330$

8462

$8 \quad 262$

$21 \quad 128$

6158

8
11

$9 \quad 345$

180

\begin{tabular}{rr}
8 & 261 \\
11 & 187 \\
\hline & 236
\end{tabular}

$\begin{array}{ll}6 & 236 \\ 3 & 340\end{array}$ 
Salary Statistics, ${ }^{1}$ 1955-56 (Group II, Cont.)

\begin{tabular}{|c|c|c|c|c|c|c|c|c|c|c|c|}
\hline \multicolumn{3}{|c|}{$\begin{array}{l}\text { All Other Profes- } \\
\text { SiONAl Assistants }\end{array}$} & \multicolumn{3}{|c|}{$\begin{array}{c}\text { AlL Nonprofessional } \\
\text { ASSISTANTS }\end{array}$} & \multicolumn{3}{|c|}{$\begin{array}{l}\text { Total Number of EMployees } \\
\text { IN FULL-TIME EQUIVALENT }\end{array}$} & \multirow{2}{*}{$\begin{array}{c}\text { Working } \\
\text { Hours in } \\
\text { Fiscal } \\
\text { Year }^{5}\end{array}$} & \multirow[b]{2}{*}{$\begin{array}{l}\text { Hours of } \\
\text { Student } \\
\text { Assistance }\end{array}$} & \\
\hline $\begin{array}{l}\text { Sio } \\
\text { Mini- } \\
\text { mum }\end{array}$ & $\begin{array}{l}\text { L Assis } \\
\text { Maxi- } \\
\text { mum }\end{array}$ & $\begin{array}{l}\text { ANTs } \\
\text { Num- } \\
\text { ber }\end{array}$ & $\underset{\text { mum }}{\text { Mini- }^{A}}$ & $\begin{array}{l}\text { SISTANTS } \\
\text { Maxi- } \\
\text { mum }\end{array}$ & $\underset{\text { ber }}{\text { Num- }}$ & $\begin{array}{l}\text { IN FULI } \\
\text { Pro- } \\
\text { fessional }\end{array}$ & $\begin{array}{l}\text {-TIME EQU } \\
\text { Nonpro- } \\
\text { fessional }\end{array}$ & $\begin{array}{l}\text { ALENT } \\
\text { Total }\end{array}$ & & & \\
\hline $\begin{array}{l}3,000 \\
3,600 \\
3,500 \\
3,600\end{array}$ & $\begin{array}{c}\ldots \ldots \\
4,140 \\
3,850 \\
\ldots \ldots\end{array}$ & $\begin{array}{l}1 \\
\because 4.5 \\
4.8\end{array}$ & $\begin{array}{l}3,000 \\
2,040 \\
2,380^{2} \\
2,400\end{array}$ & $\begin{array}{l}2,160 \\
3,100^{2} \\
3,600\end{array}$ & $\begin{array}{l}1.5 \\
4 \\
3 \\
5 \\
2.7\end{array}$ & $\begin{array}{l}3 \\
2.75 \\
5.5 \\
6 \\
2.8\end{array}$ & $\begin{array}{l}2 \\
4 \\
3 \\
4.5 \\
2.7\end{array}$ & $\begin{array}{l}5 \\
6.75 \\
8.5 \\
10.5 \\
5.5\end{array}$ & $\begin{array}{l}1,657 \\
1,762 \\
1,672 \\
1,700\end{array}$ & $\begin{array}{l}3,764 \\
3,601 \\
7,915 \\
2,679 \\
3,400\end{array}$ & 6 \\
\hline $\begin{array}{l}3,900 \\
3,000 \\
4,100 \\
1,200 \\
\ldots \ldots 2\end{array}$ & $\begin{array}{c}4,250 \\
4,300 \\
2,400 \\
\ldots .^{2}\end{array}$ & $\begin{array}{l}2 \\
8 \\
1 \\
\ddot{6}\end{array}$ & $\begin{array}{c}1,480 \\
1,800 \\
2,100 \\
\ldots \ldots 2\end{array}$ & $\begin{array}{c}2,900 \\
2,850 \\
2,520 \\
\ldots \ldots 2\end{array}$ & $\begin{array}{r}7 \\
14 \\
3 \\
7 \\
10\end{array}$ & $\begin{array}{c}5 \\
16 \\
7 \\
5.66 \\
9.5\end{array}$ & $\begin{array}{c}5.75 \\
14 \\
3 \\
5.33 \\
7.5\end{array}$ & $\begin{array}{l}10.75 \\
30 \\
10 \\
11 \\
17\end{array}$ & $\begin{array}{l}1,518 \\
1,618 \\
2,060 \\
1,200^{19} \\
1,785\end{array}$ & $\begin{array}{r}\ldots \cdots{ }^{2} \\
13,190^{2} \\
17,000 \\
5,198\end{array}$ & \\
\hline $\begin{array}{c}3,300 \\
\ldots 2^{2} \\
\cdots{ }^{2} \\
3,960\end{array}$ & $\begin{array}{l}\ldots{ }^{2} \\
\cdots{ }^{2} \\
4,080\end{array}$ & $\begin{array}{l}1 \\
2 \\
0^{2} \\
2\end{array}$ & $\begin{array}{c}1,700 \\
\cdots{ }^{2} \\
2,400^{2} \\
1,020^{9}\end{array}$ & $\begin{array}{c}2,200 \\
\cdots \cdots^{2} \\
\cdots{ }^{2} \\
2,400^{21}\end{array}$ & $\begin{array}{l}3 \\
5 \\
i^{2}\end{array}$ & $\begin{array}{l}4 \\
8 \\
7 \\
5 \\
9\end{array}$ & $\begin{array}{l}3 \\
5.5 \\
6 \\
1 \\
3\end{array}$ & $\begin{array}{l}7 \\
13.5 \\
13 \\
6 \\
12\end{array}$ & $\begin{array}{l}1,770^{20} \\
1,836 \\
1,631 \\
1,800 \\
1,755\end{array}$ & $\begin{array}{r}1,163 \\
8,023 \\
3,400 \\
8,475 \\
16,769\end{array}$ & \\
\hline $\begin{array}{l}4,200 \\
4,150 \\
3,456 \\
3,600 \\
3,900\end{array}$ & $\begin{array}{l}4,600 \\
4,500 \\
4,320 \\
3,600 \\
4,600\end{array}$ & $\begin{array}{r}3 \\
7 \\
12 \\
2 \\
9\end{array}$ & $\begin{array}{l}1,400 \\
2,288 \\
2,112 \\
1,920 \\
2,460\end{array}$ & $\begin{array}{l}1,650 \\
3,536 \\
2,880 \\
2,400 \\
3,360\end{array}$ & $\begin{array}{r}3 \\
22 \\
22 \\
7 \\
13\end{array}$ & $\begin{array}{r}4 \\
13 \\
19 \\
7 \\
14\end{array}$ & $\begin{array}{l}2.5 \\
19.5 \\
22 \\
6.66 \\
12\end{array}$ & $\begin{array}{l}6.5 \\
32.5 \\
41.5 \\
13.66 \\
26\end{array}$ & $\begin{array}{l}1,440 \\
1,700 \\
1,880 \\
1,480 \\
1,505\end{array}$ & $\begin{array}{l}8,000 \\
8,289 \\
1,569 \\
5,938 \\
864^{22}\end{array}$ & \\
\hline $\begin{array}{c}3,600 \\
\cdots \cdots{ }^{2} \\
3,800^{2} \\
3,456\end{array}$ & $\begin{array}{l}5,000 \\
\cdots \cdots{ }^{2} \\
4,200^{2} \\
4,320\end{array}$ & $\begin{array}{l}9 \\
\ddot{2}^{2} \\
3\end{array}$ & $\begin{array}{c}2,000 \\
\cdots \cdots \\
2,40{ }^{2} \\
2,304\end{array}$ & $\begin{array}{c}3,050 \\
\cdots{ }^{2} \\
3,000 \\
3,312\end{array}$ & $\begin{array}{l}10 \\
\ddot{7}^{2} \\
\ddot{7}^{2} .5\end{array}$ & $\begin{array}{l}9.5 \\
5.25 \\
3 \\
7 \\
7.5\end{array}$ & $\begin{array}{l}8.5 \\
\ddot{5} \\
6.5 \\
11\end{array}$ & $\begin{array}{c}18 \\
5.25 \\
8 \\
13.5 \\
18.5\end{array}$ & $\begin{array}{l}1,680 \\
1,864 \\
1,800 \\
1,912\end{array}$ & $\begin{array}{r}8,000 \\
\dddot{6}, 055 \\
16,394 \\
11,853\end{array}$ & \\
\hline 2,900 & $\begin{array}{l}3,200 \\
\ldots \ldots\end{array}$ & $\begin{array}{r}2 \\
\cdots^{2}\end{array}$ & 1,820 & 2,600 &.$^{4.5}$ & $\begin{array}{l}6 \\
6\end{array}$ & $\begin{array}{l}4.5 \\
1\end{array}$ & $\underset{7}{10.5}$ & $\begin{array}{l}1,638 \\
1,422\end{array}$ & $\begin{array}{l}3,349 \\
6,836\end{array}$ & \\
\hline $\begin{array}{r}4,500 \\
3,600 \\
1,200 \\
51\end{array}$ & $\begin{array}{r}6,200 \\
4,140 \\
2,400 \\
43\end{array}$ & $\begin{array}{r}12 \\
3 \\
1 \\
61\end{array}$ & $\begin{array}{r}3,000 \\
2,180 \\
1,600 \\
64\end{array}$ & $\begin{array}{r}4,575 \\
3,000 \\
1,650 \\
57\end{array}$ & $\begin{array}{c}22 \\
4.75 \\
1 \\
74\end{array}$ & $\begin{array}{c}21 \\
6 \\
1.50 \\
89\end{array}$ & $\begin{array}{l}22 \\
4.50 \\
1 \\
87\end{array}$ & $\begin{array}{l}41.50 \\
10.63 \\
4.50 \\
88\end{array}$ & $\begin{array}{r}2,080 \\
1,777 \\
1,120 \\
85\end{array}$ & $\begin{array}{r}38,457 \\
5,662 \\
864 \\
81\end{array}$ & $\begin{array}{r}\text { High } \\
\text { Median } \\
\text { Low } \\
\mathbf{N}^{23}\end{array}$ \\
\hline
\end{tabular}

Plus additional for teaching. 16. Does not include FTE for three part-time employees. 17, 1,650-1,720 according to length of service. 18. Motion picture operators only, 19. Varies from 1,200 to 1,400 . 20. Average for four positions. 21. Two-thirds time. 22. Plus 4,411 hours not on library budget. 23. Number of libraries on which high, median and low are based.

Statistics, I $955^{-1} 956$ (Group III)

\begin{tabular}{|c|c|c|c|c|c|c|c|c|c|c|}
\hline $\begin{array}{c}\text { Staff } \\
\text { Salaries }\end{array}$ & $\begin{array}{l}\text { Student } \\
\text { Service }\end{array}$ & $\begin{array}{l}\text { Total } \\
\text { Staff } \\
\text { Salaries } \\
\text { and } \\
\text { Student } \\
\text { Service }\end{array}$ & $\begin{array}{c}\text { Books } \\
\text { and } \\
\text { Related } \\
\text { Materials }\end{array}$ & Binding & $\begin{array}{l}\text { Total } \\
\text { for } \\
\text { Books } \\
\text { and } \\
\text { Binding }\end{array}$ & $\begin{array}{l}\text { Other } \\
\text { Operat- } \\
\text { ing Ex- } \\
\text { pendi- } \\
\text { tures }^{2}\end{array}$ & $\begin{array}{l}\text { Total } \\
\text { Operat- } \\
\text { ing Ex- } \\
\text { pendi- } \\
\text { tures }\end{array}$ & $\begin{array}{l}\text { Per Stu- } \\
\text { dent Op- } \\
\text { erating } \\
\text { Expendi- } \\
\text { tures }\end{array}$ & $\begin{array}{l}\text { Total Edu- } \\
\text { cational } \\
\text { and Gen- } \\
\text { eral Insti- } \\
\text { tutional } \\
\text { Expendi- } \\
\text { tures }^{3}\end{array}$ & $\begin{array}{l}\text { Ratio of } \\
\text { Library } \\
\text { Expendi- } \\
\text { tures to } \\
\text { Total } \\
\text { (Per Cent) }\end{array}$ \\
\hline $\begin{array}{r}\$ 13,200 \\
16,800 \\
13,106 \\
6,951 \\
12,510\end{array}$ & $\begin{array}{r}\$ 994 \\
1,985 \\
3,794 \\
4,245 \\
2,016\end{array}$ & $\begin{array}{r}\$ 14,914 \\
18,785 \\
16,810 \\
11,196 \\
14,526\end{array}$ & $\begin{array}{r}\$ 8,725 \\
7,590 \\
12,020 \\
5,247 \\
1,312\end{array}$ & $\begin{array}{r}\$ 1,088 \\
559 \\
1,124 \\
527 \\
561\end{array}$ & $\begin{array}{r}\$ 9,813 \\
8,149 \\
13,144 \\
5,774 \\
1,873\end{array}$ & $\begin{array}{r}\$ 1,314 \\
492 \\
1,502 \\
909 \\
454\end{array}$ & $\begin{array}{r}\$ 25,321 \\
27,426 \\
31,456 \\
17,879 \\
16,853\end{array}$ & $\begin{array}{r}\$ 44.74 \\
56.32 \\
69.21 \\
17.00 \\
17.83\end{array}$ & $\begin{array}{r}\$ 810,814 \\
871,027 \\
1,072,187 \\
539,226 \\
672,313\end{array}$ & $\begin{array}{l}3.1 \\
3.5 \\
3 . \\
3.3 \\
2.5\end{array}$ \\
\hline $\begin{array}{r}18,838 \\
10,175 \\
9,806 \\
10,021 \\
15,135\end{array}$ & $\begin{array}{r}270 \\
1,036 \\
2,285 \\
3,955 \\
1,750\end{array}$ & $\begin{array}{l}19,108 \\
11,211 \\
12,091 \\
13,976 \\
16,885\end{array}$ & $\begin{array}{l}7,317 \\
3,784 \\
8,411 \\
3,942 \\
6,800\end{array}$ & $\begin{array}{r}1,350 \\
685 \\
557 \\
1,300\end{array}$ & $\begin{array}{l}8,668 \\
4,469 \\
8,968 \\
3,942 \\
8,100\end{array}$ & $\begin{array}{r}1,344 \\
277 \\
6,582 \\
261 \\
1,300\end{array}$ & $\begin{array}{l}29,120 \\
15,957 \\
27,641 \\
18,179 \\
26,285\end{array}$ & $\begin{array}{r}11.21 \\
24.47 \\
24.88 \\
28.99 \\
123.98\end{array}$ & $\begin{array}{r}2,051,805 \\
428,186 \\
519,988 \\
268,806 \\
421,940\end{array}$ & $\begin{array}{l}1.41 \\
3.73 \\
5.3 \\
6.8 \\
6.2\end{array}$ \\
\hline $\begin{array}{r}15,696 \\
10,605 \\
2,850 \\
8,233 \\
16,142\end{array}$ & $\begin{array}{r}2,879 \\
689 \\
1,000 \\
1,340 \\
1,000\end{array}$ & $\begin{array}{r}18,575 \\
11,294 \\
3,850 \\
9,573 \\
17,142\end{array}$ & $\begin{array}{l}8,082 \\
4,588 \\
4,276 \\
4,595 \\
3,781\end{array}$ & $\begin{array}{r}1,500 \\
412 \\
168 \\
550 \\
922\end{array}$ & $\begin{array}{l}9,582 \\
5,000 \\
4,444 \\
5,145 \\
4,703\end{array}$ & $\begin{array}{r}1,456 \\
592 \\
177 \\
399 \\
169\end{array}$ & $\begin{array}{r}29,614 \\
16,886 \\
8,471 \\
15,117 \\
22,014\end{array}$ & $\begin{array}{l}35.60 \\
29.93 \\
47.86 \\
35.32 \\
64.37\end{array}$ & $\begin{array}{l}382,441 \\
182,711 \\
310,736 \\
531,642\end{array}$ & $\begin{array}{l}4.5 \\
4.6 \\
4.8 \\
4.14\end{array}$ \\
\hline $\begin{array}{r}5,586 \\
13,540 \\
12,158 \\
5,400 \\
8,230\end{array}$ & $\begin{array}{r}982 \\
3,602 \\
1,052 \\
530 \\
3,033\end{array}$ & $\begin{array}{r}6,568 \\
17,142 \\
13,210 \\
5,930 \\
11,263\end{array}$ & $\begin{array}{l}2,000 \\
7,186 \\
3,515 \\
2,990 \\
5,099\end{array}$ & $\begin{array}{r}75 \\
1,500 \\
742 \\
796\end{array}$ & $\begin{array}{l}2,075 \\
8,686 \\
4,257 \\
2,990 \\
5,564\end{array}$ & $\begin{array}{c}1,500 \\
1,367 \\
\ldots \ldots \\
\ddot{504}\end{array}$ & $\begin{array}{r}10,143 \\
27,195 \\
18,211 \\
8,920 \\
17,331\end{array}$ & $\begin{array}{l}34.85 \\
26.04 \\
29.00 \\
20.66 \\
48.30\end{array}$ & $\begin{array}{c}272,400 \\
\ldots \ldots \\
3 \ddot{3} 6,954 \\
513,934\end{array}$ & $\begin{array}{l}3.33 \\
\cdots \\
\cdots \\
3.4\end{array}$ \\
\hline $\begin{array}{r}15,600 \\
5,400 \\
4,000 \\
13,895 \\
16,244\end{array}$ & $\begin{array}{l}1,850 \\
1,147 \\
3,075 \\
6,707 \\
1,986\end{array}$ & $\begin{array}{r}17,450 \\
6,547 \\
7,075 \\
20,602 \\
18,230\end{array}$ & $\begin{array}{l}6,000 \\
2,414 \\
2,750 \\
9,565 \\
4,450\end{array}$ & $\begin{array}{r}1,000 \\
96 \\
375 \\
1,395 \\
950\end{array}$ & $\begin{array}{r}7,000 \\
2,510 \\
3,125 \\
10,960 \\
5,400\end{array}$ & $\begin{array}{r}2,950 \\
169 \\
200 \\
1,235 \\
1,022\end{array}$ & $\begin{array}{r}26,600 \\
9,326 \\
10,400 \\
32,797 \\
24,652\end{array}$ & $\begin{array}{l}20.14 \\
37.91 \\
41.76 \\
31.59 \\
21.57\end{array}$ & $\begin{array}{l}862,496 \\
217,338 \\
207,211 \\
832,172 \\
468,885\end{array}$ & $\begin{array}{l}3 . \\
4.2 \\
5 . \\
3.9 \\
5.2\end{array}$ \\
\hline
\end{tabular}




\section{Library $^{1}$}

Fiscal

Ending

26. Concordia (Minn.) ......... 30 Je56

27. Drury (Mo.) .............. $31 \mathrm{My} 56$

28. Earlham (Ind.) $\ldots \ldots \ldots \ldots \ldots, 30$. 3056

29. Elon (N.C.) .............. $31 \mathrm{My} 56$

30. Emory and Henry (Va.) ..... 31Ag56

31. Evansville (Ind.) $\ldots \ldots \ldots \ldots \ldots$ 30 Je56

32. Fenn (Ohio) $\ldots \ldots \ldots \ldots \ldots \ldots, \ldots$

33. Findlay (Ohio)

34. Franklin (Ind.) .................

35. Geneva (Pa.) ...

$31 \mathrm{Ag} 56$

30 Ap56

$30 \mathrm{Je} 56$
$30 \mathrm{Je} 56$

$30 \mathrm{Je} 56$

36. Georgia State Coll. for Women 37. Gettysburg $(\mathrm{Pa}.) \ldots \ldots \ldots \ldots \ldots$

38. Golden Gate (Calif.) $\ldots \ldots \ldots \ldots$

40. Gustavus Adolphus (Minn.) ....

41. Hamlin (Minn.) (Y............

42. Hampden-Sydney (Va.) $\ldots \ldots$.

44. Heidelberg (Ohio) $\ldots \ldots \ldots, \ldots$

45. Hendrix (Ark.)

46. Hillsdale (Mich.) $\ldots \ldots \ldots \ldots \ldots$

47. Hiram (Ohio) $\ldots \ldots \ldots \ldots \ldots \ldots \ldots .$.

48. Hollins $\mathrm{Va}$.

49. Hood (Md.) $\ldots \ldots \ldots \ldots \ldots \ldots \ldots \ldots \ldots$

51. Houghton (N.Y.)

52. Huntingdon (Ala.) .............

(Ind.) ........... $31 \mathrm{Jy5} 56$

54. Illinois College $\ldots \ldots \ldots \ldots \ldots \ldots, 30 \mathrm{~J}$ e56

55. Illinois Wesleyan ............

56. Iowa Wesleyan ............ $31 \mathrm{Ag} 56$

$\begin{array}{lll}\text { 57. Jamestown (N.D.) } \quad \ldots \ldots \ldots \ldots \ldots & \text { 30Je56 } \\ 30 \mathrm{Je} 56\end{array}$

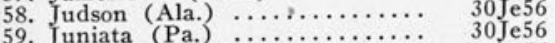

60. Lafayette $($ Pa. $) \ldots \ldots \ldots \ldots \ldots, \quad 31 \mathrm{Ag} 56$

61. LaGrange (Ga.) ........... 30 Je56

62. Lake Forest (Iil.) ........... 30 3056

63. LaVerne (Calif.) ............. $31 \mathrm{Ag} 56$

64. Lawrence (Wis.) ............ 30 3056

65. Lebanon Valley (Pa.) $\cdots \cdots \cdots \cdots, 30$ p 56

66. Livingstone (N.C.) $\ldots \ldots \ldots \ldots$ 30 3056

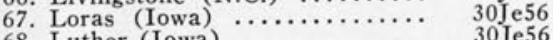

68. Luther (Iowa) $\ldots \ldots \ldots \ldots \ldots \ldots, \quad \begin{aligned} & 30 \mathrm{Je} 56 \\ & 30 \mathrm{Je} 56\end{aligned}$

70. Madison (Tenn.) ........... 31Ag56

71. Manchester (Ind.) $\ldots \ldots \ldots \ldots \ldots$ 31Jy56

72. Marietta (Ohio) ............. 30 30 56

72. Marietta (Ohio) $\ldots \cdots \cdots \cdots \cdots, 31 \mathrm{My} 56$

74. Marymount (Calif.) $\ldots \ldots \ldots \ldots \ldots$
75. Marywood (Pa.)...$\cdots \cdots \cdots \cdots$

76. Meredith (N.C.) $\ldots \ldots \ldots \ldots \ldots \ldots$ 30Je56

77. Midland (Neb.) $\ldots \ldots \ldots \ldots \ldots \ldots, 31 \mathrm{My} 56$

78. Millikin (III.) $\ldots \ldots \ldots \ldots \ldots \ldots, 30 \mathrm{Je} 56$

80. Mount Union (Ohio) ........... 30 Je56

81. Muskingum (Ohio) ......... 31Ag56

82. Nebraska Wesleyan .......... 30 My56

83. New Mexico Highlands ...... 30 Je56

84. Pacific Union (Calif.) $\ldots \ldots \ldots \ldots .$.
85. Paine (Ga.)

86. Parsons (Iowa) ............ $31 \mathrm{Ag} 56$

87. Pepperdine (Calif.) $\ldots \ldots \ldots \ldots .31 \mathrm{Ag} 56$

88. Randolph-Macon (Va.) ....... 30Ap56

89. Ripon (Wis.) .............. 30 Je56

90. Rosary (III.) …............ $30 \mathrm{Je} 56$

91. Saint Joseph's College (Ind.) . 31Jy56

92. Savannah (Ga.) …........ 30 3056

93. Scripps (Calif.) $\ldots \ldots \ldots \ldots \ldots \ldots$ 30 3056

94. Seton Hill (Pa.) $\cdots \ldots \ldots \ldots \ldots . .6 \%$ 31Ag56

96. Springfield (Mass.) ......... 31Ag56

97. Talladega (Ala) ............ 30 30 e56

98. Tennessee Wesleyan ............. $31 \mathrm{Ag} 56$

98. Tennessee Wesleyan ........... 31 30Je56

99. Trinity (D.C.) $\ldots \ldots \ldots \ldots \ldots \ldots$
100. Tusculum (Tenn.)
Student EnROLLMENT

Total

Under. Total Total

graduate Graduate Extension Stock

$\begin{array}{rrrr}1,354 & & \ldots & 47,533 \\ 619 & 14 & \ldots & 38,543 \\ 737 & 3 & 256 & 86,854 \\ 853 & \ldots & \ldots & 45,585 \\ 517 & \ldots & \ldots & 35,146\end{array}$

Volumes

$2,879 \quad 10 \quad 292$

1,300

$2,062^{4}$

1,552

1,654

1,274

2,548

528

834

196

632

1,403

1,591

1,020

1,230

360
739

739
638

417

591

560

567

486

876

592

640
203

360

1,002

520

441

248

668
1,536

213

708

788

592

529

1,020

1,039
763

404

906

906
851
716

144

613

39

1,136

670

857

857
858
724

858
724

772
277

324

898

675

555

656

815

951
$207^{6}$

$207^{6}$
491

584

899

314
447

486

253
40,386

$\begin{array}{ll}\cdots & 35,489\end{array}$

$1,900^{4}$

1,793

1,177

$1,548^{4}$

$2,657^{4}$

2,6344
1,367

1,367
1,400

1,400

$3,054^{4}$

$1,723^{4}$

1,941

1,941
$1,199^{4}$

$675^{4}$

3,060

$1,921^{4}$

, 255

44,497

36,838

36,838
46,759

46,759
20,886

20,886
44,569

1,815

1,815
2,026

1,399

1,395

52,000

717

$24,000^{3}$

25,959

21,861

142,414

$1,633^{4}$

1,048

$2,330^{4}$

17,411

107

535

1,708

$1,448^{4}$

1,992

75,758

58,519

34,549

119,044

104,510

31,247
24,027

720

2,000

$3,102^{4}$
$1,174^{4}$

486
486

45,270

1,427

$2,080^{4}$

1,851

16,435

38,503

$1,056^{4}$

$1,552^{4}$

1,004

2,550

1,900

877
3,8494

3,849

1,732
574

$\begin{array}{rrr}43 & 20 & 65,584 \\ 13 & \because 69 & 47,829 \\ \ldots & 69,835\end{array}$

2,032

33,080
52,007

52,007
80,599

2,088

$1,633^{4}$

2,653

74,630

3,326

62,944

23,043

$1,071^{4}$

1,669

43,063

1,126

47,340

$1,622^{4}$

$1,090^{4}$

$1,899^{4}$

21,153

70,085

3,037
690
News- Peri-

$6 \quad 378$

$17 \quad 385$

9290

$4 \quad 464$

$13 \quad 257$

$11 \quad 276$

3
5 156

$\begin{array}{ll}8 & 287 \\ 7 & 292\end{array}$

$\begin{array}{ll}7 & 318\end{array}$

261

293

325
308

181

435
287

290

285

282

342

148
202

283

156

325

168

371

160

351
210

400

391

153

262

262
237

256

312

392

231

299

239

272

253
480

520

304

188

325

480
145

192

475
337

337
$304^{5}$

448

582

278 
Statistics, $1955^{-5} 6$ (Group III, Cont.)

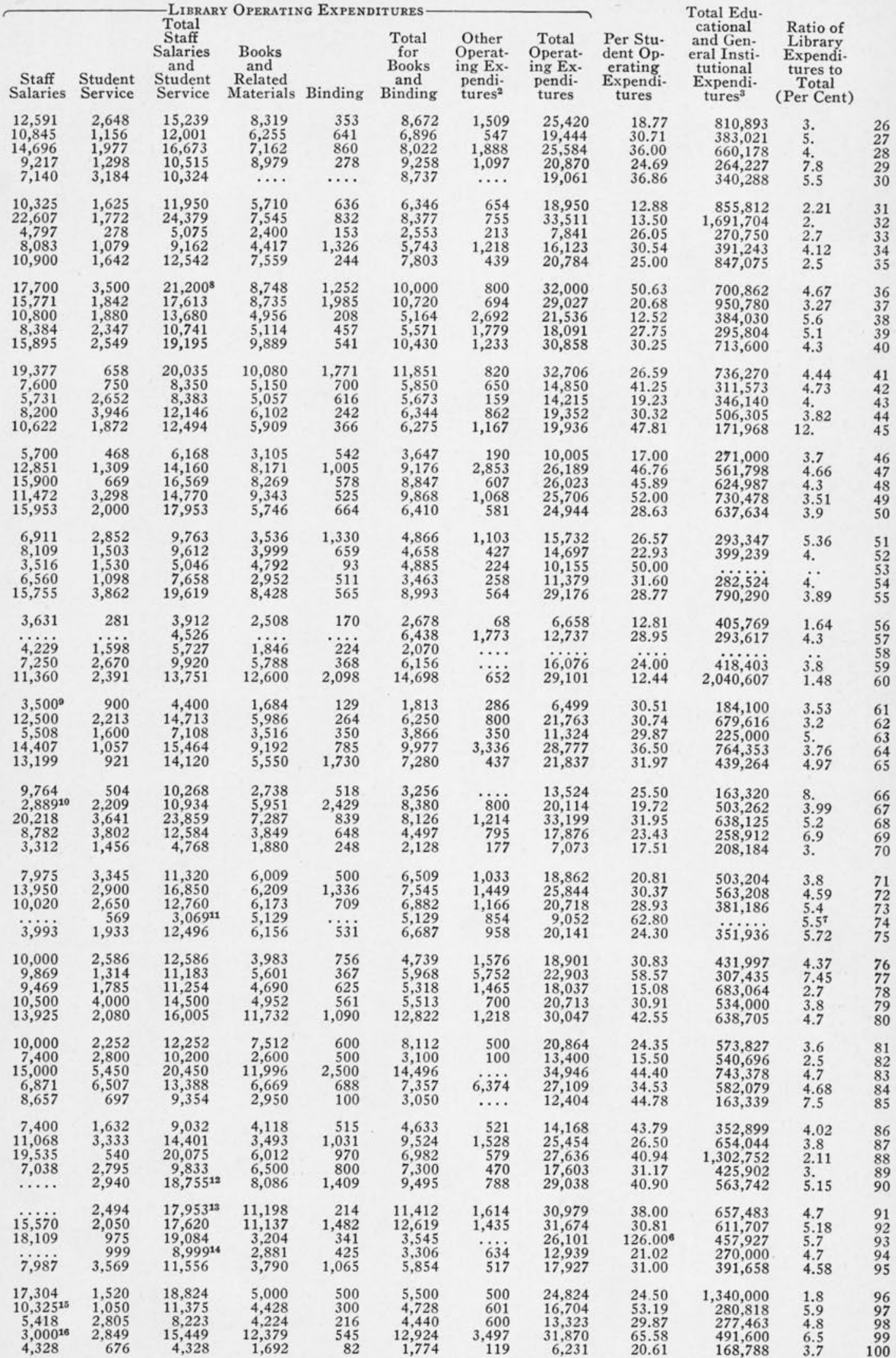




\begin{tabular}{|c|c|c|c|c|c|c|c|c|}
\hline brary $^{1}$ & $\begin{array}{l}\text { Fiscal } \\
\text { Year } \\
\text { Ending }\end{array}$ & $\begin{array}{l}\text { STuDE } \\
\text { Total } \\
\text { Under- } \\
\text { graduate }\end{array}$ & $\begin{array}{l}\text { NT ENROLI } \\
\text { Total } \\
\text { Graduate }\end{array}$ & $\begin{array}{l}\text { LMENT } \\
\text { Total } \\
\text { Extension }\end{array}$ & $\begin{array}{c}\text { Book } \\
\text { Stock }\end{array}$ & $\begin{array}{l}\text { Volumes } \\
\text { Added }\end{array}$ & $\begin{array}{l}\text { News- } \\
\text { papers }\end{array}$ & $\begin{array}{c}\text { Peri- } \\
\text { odicals }\end{array}$ \\
\hline 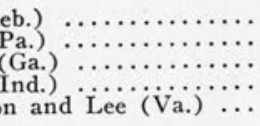 & $\begin{array}{r}30 \mathrm{Je} 56 \\
30 \mathrm{Je} 56 \\
31 \mathrm{My} 56 \\
31 \mathrm{Ag} 56 \\
30 \mathrm{Je} 56\end{array}$ & $\begin{array}{l}779 \\
720 \\
460 \\
589 \\
960\end{array}$ & $\begin{array}{l}\cdots \\
\cdots \\
\cdots \\
\cdots\end{array}$ & $\begin{array}{l}\cdots \\
\cdots \\
\cdots \\
\cdots\end{array}$ & $\begin{array}{r}55,939 \\
46,009 \\
34,531 \\
100,000 \\
166,091\end{array}$ & $\begin{array}{l}1,826 \\
1,301^{4} \\
1,431 \\
4,500^{4} \\
3,563\end{array}$ & $\begin{array}{r}7 \\
9 \\
10 \\
4 \\
11\end{array}$ & $\begin{array}{l}378 \\
224 \\
238 \\
411 \\
412\end{array}$ \\
\hline 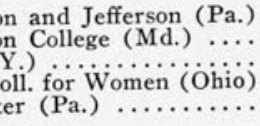 & $\begin{array}{l}30 \mathrm{Je} 56 \\
30 \mathrm{Je} 56 \\
30 \mathrm{Je} 56 \\
30 \mathrm{Je} 56 \\
31 \mathrm{Jy} 56\end{array}$ & $\begin{array}{l}635 \\
467 \\
367 \\
294 \\
897\end{array}$ & $\begin{array}{r}8 \\
\dddot{1} \\
17 \dot{8}\end{array}$ & $\begin{array}{l}\cdots \\
\cdots \\
\cdots \\
\cdots\end{array}$ & $\begin{array}{c}88,757 \\
47,111 \\
120,000^{7} \\
51,023 \\
52,948\end{array}$ & $\begin{array}{l}1,568 \\
1,061^{4} \\
2,243 \\
1,250 \\
2,528\end{array}$ & $\begin{array}{r}8 \\
7 \\
7 \\
5 \\
20\end{array}$ & $\begin{array}{l}212 \\
215 \\
362 \\
250 \\
291\end{array}$ \\
\hline $\begin{array}{l}\text { alif.) } \cdots \cdots \cdots \cdots \\
\text { (Ohio) } \\
\text { (Oreg.) }\end{array}$ & $\begin{array}{r}31 \mathrm{Ag} 56 \\
30 \mathrm{Ap} 56 \\
30 \mathrm{Je} 56\end{array}$ & $\begin{array}{r}992 \\
248 \\
1,078\end{array}$ & $\begin{array}{r}78 \\
11 \\
7\end{array}$ & $\begin{array}{l}\cdots \\
\cdots \\
\cdots\end{array}$ & $\begin{array}{l}58,719 \\
38,285 \\
56,757\end{array}$ & $\begin{array}{l}1,770 \\
2,279 \\
1,646\end{array}$ & $\begin{array}{r}75 \\
8\end{array}$ & $\begin{array}{l}458 \\
296 \\
336\end{array}$ \\
\hline$\cdots \cdots$ & & $\begin{array}{r}2,548 \\
619 \\
144 \\
113\end{array}$ & $\begin{array}{r}196 \\
16 \\
1 \\
28\end{array}$ & $\begin{array}{r}2,081 \\
77 \\
11 \\
21\end{array}$ & $\begin{array}{r}166,091 \\
46,009 \\
16,435 \\
112\end{array}$ & $\begin{array}{r}4,500 \\
1,633 \\
180 \\
112\end{array}$ & $\begin{array}{r}75 \\
8 \\
3 \\
113\end{array}$ & $\begin{array}{r}582 \\
290 \\
96 \\
113\end{array}$ \\
\hline
\end{tabular}

1. Central library and all agencies. 2. Includes supplies, transportation, and all other non-capital expenditures 1. Central ally reported. 3. Total expenditures for educational and general purposes include administration, resinot specifically reported. capital costs and expenditures for auxiliary enterprises such as dormitories, cafeterias, etc. 4. Includes docucapital costs and expenditures for auxilary enterprises service to students and faculty of other Associated Col-

\section{College and University Library Salary}

\begin{tabular}{|c|c|c|c|c|c|c|c|c|c|}
\hline Library $^{1}$ & $\underset{\text { brarian }}{\text { Li- }}$ & $\begin{array}{l}\text { Assoc } \\
\text { Assis } \\
\text { CHIEF L } \\
\text { Mini- } \\
\text { mum }\end{array}$ & $\begin{array}{l}\text { IATE OR } \\
\text { STANT } \\
\text { IBRARIAN } \\
\text { MaXi- } \\
\text { mum }\end{array}$ & $\begin{array}{l}\text { DEPAR } \\
\text { Mini- } \\
\text { mum }\end{array}$ & $\begin{array}{l}\text { MENT H } \\
\text { Maxi- } \\
\text { mum }\end{array}$ & $\begin{array}{l}\text { EADS } \\
\text { ber }\end{array}$ & $\begin{array}{l}\text { ALL OT } \\
\text { SIONAL } \\
\text { Mini- } \\
\text { mum }\end{array}$ & $\begin{array}{l}\text { HER PRo } \\
\text { ASSISTA } \\
\text { Maxi- } \\
\text { mum }\end{array}$ & $\begin{array}{l}\text { FEs- } \\
\text { NTS } \\
\text { Num- } \\
\text { ber }\end{array}$ \\
\hline $\begin{array}{l}\text { 1. Agnes Scott (Ga.) } \ldots \ldots \ldots \ldots \ldots \\
\text { 2. Alabama College } \ldots \ldots \ldots \ldots \ldots \ldots \\
\text { 3. Alaska . . } \ldots \ldots \ldots \ldots \\
\text { 4. Anderson (Ind.) } \ldots \ldots \ldots \ldots \ldots \\
\text { 5. Arkansas A. \& M. } \ldots \ldots \ldots \ldots \ldots\end{array}$ & $\begin{array}{l}\$ \ldots 4 \\
7,800 \\
3,900 \\
4,410\end{array}$ & $\begin{array}{l}\$ \ldots 4 \\
\ldots{ }^{4} \\
\ldots \\
\ddot{3}, \dot{9} \dot{0}\end{array}$ & $\begin{array}{l}\$ \ldots \\
\ldots \\
\ldots \\
\ldots \\
\ldots\end{array}$ & $\begin{array}{l}\$ \ldots \\
\ldots \\
\ldots \\
\ldots \\
\ldots \\
\cdots\end{array}$ & $\begin{array}{l}\$ \ldots \\
\ldots \\
\ldots \\
\ldots \\
\ldots\end{array}$ & $\begin{array}{l}\cdots \\
\cdots \\
\cdots \\
\cdots\end{array}$ & $\begin{array}{l}\$ \ldots \\
\ldots{ }^{4} \\
\ldots \\
\ldots \\
\ldots{ }^{4}\end{array}$ & $\begin{array}{l}\$ \beta^{4} \\
\ldots{ }^{4} \\
\ldots \\
\ldots \\
\cdots\end{array}$ & $\begin{array}{l}\cdots^{4} \\
\cdots \\
\cdots \\
\cdots\end{array}$ \\
\hline 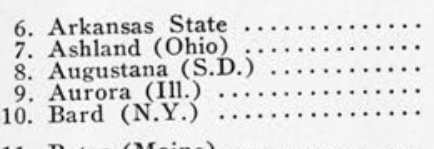 & $\begin{array}{l}5,400^{4} \\
4,520 \\
\ldots \ldots \\
\cdots\end{array}$ & $\ddot{\cdots}^{4}$ & $\begin{array}{l}\ldots{ }^{4} \\
\ldots{ }^{4} \\
\cdots 4^{4} \\
\cdots\end{array}$ & $\begin{array}{l}\cdots{ }^{4} \\
\cdots \\
\cdots \\
\cdots{ }^{4}\end{array}$ & $\begin{array}{l}\cdots{ }^{4} \\
\cdots \cdots \\
\cdots \cdots \\
\cdots\end{array}$ & $\begin{array}{l}\cdots^{4} \\
\cdots \\
\cdots 4\end{array}$ & $\begin{array}{l}\ldots{ }^{4} \\
\ldots{ }^{4} \\
3 \ddot{7} \dot{8} 0 \\
\ldots{ }^{4}\end{array}$ & $\begin{array}{l}\cdots{ }^{4} \\
\cdots \\
\cdots \\
\cdots\end{array}$ & $\begin{array}{l}\ddot{2}^{4} \\
\dot{3}_{4}^{4}\end{array}$ \\
\hline 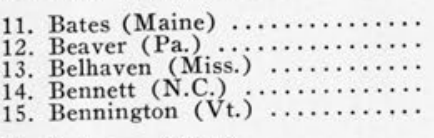 & $\begin{array}{l}5,600 \\
4,200 \\
2,850 \\
\ldots \ldots \\
\ldots \ldots\end{array}$ & $\begin{array}{c}3,000 \\
3,400 \\
\ldots \\
\ldots \cdots \\
\cdots\end{array}$ & $\begin{array}{l}3,500 \\
\ldots \\
\ldots \\
\cdots \\
\cdots\end{array}$ & $\begin{array}{l}\cdots \cdots \\
\cdots \\
\cdots \\
\cdots\end{array}$ & $\begin{array}{l}\cdots \\
\cdots \\
\cdots \\
\cdots\end{array}$ & $\begin{array}{l}\ldots \\
{ }_{1.25}\end{array}$ & $\begin{array}{l}\ldots \\
\ldots \\
\cdots \\
\cdots \\
\cdots\end{array}$ & $\begin{array}{l}\cdots \cdots \\
\cdots \cdots \\
\cdots \cdots \\
\cdots\end{array}$ & $\begin{array}{l}{ }^{4} \\
\ldots \\
\ldots\end{array}$ \\
\hline 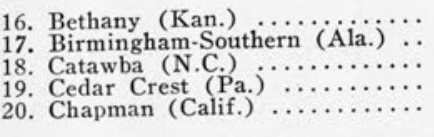 & $\begin{array}{l}3,500 \\
4,700 \\
4,800^{1} \\
4,100 \\
4,560\end{array}$ & $\begin{array}{l}\cdots \\
3,300^{5} \\
\ddot{3}, \dot{4} \dot{7} 0\end{array}$ & $\begin{array}{l}\cdots \\
\cdots \\
\cdots \\
\cdots\end{array}$ & $\begin{array}{l}3 \ddot{3} \dot{50} \\
3,100 \\
\cdots \\
\cdots\end{array}$ & $\begin{array}{c}3,350 \\
\ldots \\
\cdots \\
\cdots\end{array}$ & $\begin{array}{l}3 \\
1 \\
\ldots \\
\cdots\end{array}$ & $\begin{array}{l}\cdots \cdots \\
\cdots \\
\cdots \\
900^{6}\end{array}$ & $\begin{array}{l}\cdots \\
\cdots \\
\cdots \\
\cdots \\
\cdots\end{array}$ & $\begin{array}{l}\cdots \\
\therefore \\
\cdots\end{array}$ \\
\hline 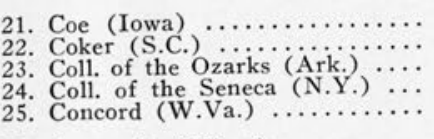 & $\begin{array}{l}5,200 \\
3,000 \\
4,000 \\
4,900 \\
5,320\end{array}$ & $\begin{array}{l}\cdots \\
\cdots \\
\cdots \\
\cdots \\
\cdots\end{array}$ & $\begin{array}{l}\cdots \\
\cdots \\
3,850 \\
5,196\end{array}$ & $\begin{array}{l}\cdots \\
\cdots \\
\cdots \\
\cdots\end{array}$ & $\begin{array}{l}\cdots \\
\cdots \\
3,600 \\
\cdots\end{array}$ & $\begin{array}{l}\cdots \\
i \\
\cdots\end{array}$ & $\begin{array}{l}3,600 \\
2,400 \\
\ldots \\
\cdots \\
\cdots\end{array}$ & $\begin{array}{l}4,100 \\
\cdots \\
\cdots \\
\cdots \\
\cdots\end{array}$ & $\begin{array}{l}3 \\
\cdots \\
\cdots \\
\cdots\end{array}$ \\
\hline $\begin{array}{l}\text { 26. Concordia (Minn.) } \ldots \ldots \ldots \ldots \ldots \\
\text { 27. Drury (Mo.) } \ldots \ldots \ldots \ldots \ldots \ldots \\
\text { 28. Earlham (Ind.) } \ldots \ldots \ldots \ldots \ldots \ldots \\
\text { 29. Elon (N.C.) } \ldots \ldots \ldots \ldots \ldots \\
\text { 30. Emory and Henry }(\text { Va. }) \ldots \ldots\end{array}$ & $\begin{array}{l}4,200 \\
4,300 \\
5,500 \\
3,400 \\
4,500\end{array}$ & $\begin{array}{l}3,700 \\
3,000 \\
4,000 \\
2,700 \\
3,000\end{array}$ & $\begin{array}{c}3,650 \\
2,700^{8} \\
\ldots\end{array}$ & $\begin{array}{l}\cdots \cdots \\
\cdots \\
\cdots \cdots \\
\cdots\end{array}$ & $\begin{array}{l}\cdots \\
\cdots \\
\cdots \\
\cdots \\
\cdots\end{array}$ & $\begin{array}{l}\cdots \\
\cdots \\
\cdots\end{array}$ & $\begin{array}{l}\cdots \\
3,600 \\
\cdots\end{array}$ & $\begin{array}{l}\cdots \\
4,000 \\
\cdots \\
\cdots\end{array}$ & $\begin{array}{l}\cdots \\
i \\
\cdots \\
\cdots\end{array}$ \\
\hline $\begin{array}{l}\text { 31. Evansville (Ind.) } \ldots \ldots \ldots \ldots \ldots \ldots \\
\text { 32. Fenn (Ohio) } \ldots \ldots \ldots \ldots \ldots \ldots \\
\text { 33. Findlay (Ohio) } \ldots \ldots \ldots \ldots \ldots \ldots \\
\text { 34. Franklin (Ind.) } \ldots \ldots \ldots \ldots \ldots \ldots \\
\text { 35. Geneva (Pa.) } \ldots \ldots \ldots \ldots \ldots\end{array}$ & $\begin{array}{l}5,000 \\
4,090 \\
5,300 \\
4,800\end{array}$ & $\begin{array}{c}4,300 \\
\ldots \ldots \\
3,900 \\
4,180\end{array}$ & $\begin{array}{l}\cdots \\
\cdots \\
\cdots \\
\cdots\end{array}$ & $\begin{array}{l}\ldots \\
\cdots \\
\cdots \\
\cdots\end{array}$ & $\begin{array}{l}\cdots \\
\cdots \\
\cdots \\
\cdots\end{array}$ & $\begin{array}{l}\cdots \\
\cdots \\
\cdots\end{array}$ & $\begin{array}{c}3,600 \\
\ldots \\
3,400\end{array}$ & $\begin{array}{l}\ldots \\
\cdots \cdots \\
\cdots \\
\cdots \\
\ldots\end{array}$ & $\begin{array}{l}\ddot{3} \\
\cdots \\
\dot{1}\end{array}$ \\
\hline $\begin{array}{l}\text { 36. Georgia State Coll. for Women } \\
\text { 37. Gettysburg (Pa.) } \ldots \ldots \ldots \ldots \ldots \\
\text { 38. Golden Gate (Calif.) } \ldots \ldots \ldots \ldots \\
\text { 39. Guilford (N.C.) } \ldots \ldots \ldots \ldots \\
\text { 40. Gustavus Adolphus (Minn.) } \ldots\end{array}$ & $\begin{array}{l}5,800 \\
5,104 \\
6,600^{9} \\
2,800^{6} \\
5,407\end{array}$ & $\begin{array}{l}3,900^{5} \\
3,733 \\
4,200 \\
3,000 \\
\ldots\end{array}$ & $\begin{array}{l}4,000^{5} \\
\cdots \\
3,300 \\
\cdots\end{array}$ & $\begin{array}{c}3,533 \\
\ldots \ldots \\
4,57 i\end{array}$ & $\begin{array}{l}\cdots \\
\cdots \\
\vdots \\
5,9 \\
\end{array}$ & $\begin{array}{l}i \\
\cdots \\
2\end{array}$ & $\begin{array}{c}\cdots \\
3,600 \\
\ldots \\
\ldots\end{array}$ & $\begin{array}{l}\ldots \ldots \\
\ldots \\
\ldots \\
\cdots \\
\cdots\end{array}$ & $\begin{array}{l}\cdots \\
i \\
\cdots\end{array}$ \\
\hline 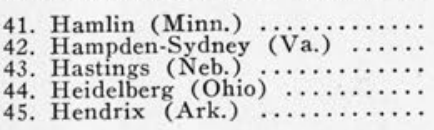 & $\begin{array}{l}6,400 \\
4,900 \\
4,200 \\
4,900 \\
3,600\end{array}$ & $\begin{array}{l}\ldots \ldots \\
3 \ldots \\
3,900 \\
2,600\end{array}$ & $\begin{array}{l}\ldots \\
\ldots \\
\ldots \\
\ldots \\
\ldots\end{array}$ & $\begin{array}{l}2,700 \\
\ldots \\
\ldots \\
\ldots \\
\ldots\end{array}$ & $\begin{array}{l}3,700 \\
\ldots \\
\cdots \\
\cdots \\
\cdots\end{array}$ & $\begin{array}{l}2 \\
\cdots \\
\cdots \\
\cdots\end{array}$ & $\begin{array}{c}1,350^{6} \\
2,700 \\
\cdots \\
1,800\end{array}$ & $\begin{array}{l}\ldots \\
\cdots \\
\cdots \\
\cdots \\
\cdots\end{array}$ & $\begin{array}{l}2 \\
1 \\
\cdots \\
1\end{array}$ \\
\hline
\end{tabular}


Statistics, $1955^{-5} 6$ (Group III, Cont.)

\begin{tabular}{|c|c|c|c|c|c|c|c|c|c|c|c|}
\hline $\begin{array}{l}\text { Staff } \\
\text { Salaries }\end{array}$ & $\begin{array}{l}\text { Studen } \\
\text { Servic }\end{array}$ & $\begin{array}{l}\text { Total } \\
\text { Staff } \\
\text { Salaries } \\
\text { and } \\
\text { Student } \\
\text { Service }\end{array}$ & $\begin{array}{l}\text { Books } \\
\text { and } \\
\text { Related } \\
\text { Materials }\end{array}$ & Binding & $\begin{array}{c}\text { Total } \\
\text { for } \\
\text { Books } \\
\text { and } \\
\text { Binding }\end{array}$ & $\begin{array}{l}\text { Other } \\
\text { Operat- } \\
\text { ing Ex- } \\
\text { pendi- } \\
\text { tures }^{2}\end{array}$ & $\begin{array}{l}\text { Total } \\
\text { Operat- } \\
\text { ing Ex- } \\
\text { pendi- } \\
\text { tures }\end{array}$ & $\begin{array}{c}\text { Per Stu- } \\
\text { dent Op- } \\
\text { erating } \\
\text { Expendi- } \\
\text { tures }\end{array}$ & $\begin{array}{l}\text { Total Edu- } \\
\text { cational } \\
\text { and Gen- } \\
\text { eral Insti- } \\
\text { tutional } \\
\text { Expendi- } \\
\text { tures }^{3}\end{array}$ & $\begin{array}{l}\text { Ratio-of } \\
\text { Library } \\
\text { Expendi- } \\
\text { tures to } \\
\text { Total } \\
\text { (Per Cent) }\end{array}$ & \\
\hline $\begin{array}{r}4,998 \\
10,787 \\
8,100 \\
16,200 \\
21,290\end{array}$ & $\begin{array}{l}8,402 \\
2,461 \\
1,697 \\
2,826\end{array}$ & $\begin{array}{r}13,400 \\
13,248 \\
9,797 \\
19,026 \\
22,490\end{array}$ & $\begin{array}{r}4,298 \\
4,798 \\
4,169 \\
10,103 \\
10,214\end{array}$ & $\begin{array}{r}1,544 \\
349 \\
515 \\
2,067 \\
1,444\end{array}$ & $\begin{array}{r}5,842 \\
5,147 \\
4,684 \\
12,170 \\
11,758\end{array}$ & $\begin{array}{r}1,674 \\
294 \\
96 \\
2,655 \\
738\end{array}$ & & & $\begin{array}{c}473,649 \\
282,963 \\
650,000^{7} \\
1,026,836\end{array}$ & $\begin{array}{l}4.42 \\
4.87 \\
5.2 \\
3.4\end{array}$ & \\
\hline $\begin{array}{r}12,600 \\
10,100 \\
17,836 \\
8,300 \\
14,200\end{array}$ & $\begin{array}{r}2,000 \\
1,300 \\
975 \\
1,445 \\
3,162\end{array}$ & $\begin{array}{c}14,600 \\
11,900^{17} \\
18,811 \\
9,975^{18} \\
17,162\end{array}$ & $\begin{array}{r}6,200 \\
3,205 \\
10,562 \\
4,488 \\
7,638\end{array}$ & $\begin{array}{r}450 \\
223 \\
1,156 \\
523 \\
534\end{array}$ & $\begin{array}{r}6,650 \\
3,528 \\
11,718 \\
5,011 \\
8,172\end{array}$ & $\begin{array}{r}1,200 \\
286 \\
1,657 \\
457 \\
1,320\end{array}$ & & $\begin{array}{l}36.00 \\
33.06 \\
90.00 \\
52.52 \\
24.97\end{array}$ & $\begin{array}{r}542,000 \\
412,533 \\
487,676 \\
607,063\end{array}$ & $\begin{array}{l}4 . \\
3.1 \\
7 . \\
4.4\end{array}$ & \\
\hline $\begin{array}{r}12,088 \\
5,156 \\
12,700\end{array}$ & $\begin{array}{l}5,523 \\
3,448 \\
3,625\end{array}$ & $\begin{array}{r}17,611 \\
8,604 \\
16,325\end{array}$ & $\begin{array}{l}6,500 \\
5,088 \\
6,867\end{array}$ & $\begin{array}{r}1,493 \\
\dddot{800}\end{array}$ & $\begin{array}{l}7,993 \\
5,088 \\
7,667\end{array}$ & $\begin{array}{r}612 \\
7300\end{array}$ & & $\begin{array}{c}24.59 \\
53.00 \\
\ldots \ldots\end{array}$ & $\begin{array}{c}1,150,000^{7} \\
333,409 \\
651,440\end{array}$ & $\begin{array}{l}2.3 \\
4.1 \\
3.8\end{array}$ & \\
\hline $\begin{array}{r}22,607 \\
10,175 \\
2,850 \\
108\end{array}$ & $\begin{array}{r}8,402 \\
1,880 \\
270 \\
112\end{array}$ & $\begin{array}{r}24,379 \\
12,542 \\
3,069 \\
113\end{array}$ & $\begin{array}{r}12,600 \\
5,710 \\
1,312 \\
111\end{array}$ & $\begin{array}{r}2,500 \\
565 \\
75 \\
107\end{array}$ & $\begin{array}{r}14,698 \\
6,275 \\
1,813 \\
113\end{array}$ & $\begin{array}{r}6,582 \\
788 \\
68 \\
103\end{array}$ & $\begin{array}{r}34,986 \\
20,784 \\
6,231 \\
112\end{array}$ & $\begin{array}{r}123.98 \\
30.19 \\
11.21 \\
110\end{array}$ & $\begin{array}{r}2,051,805 \\
491,600 \\
163,320 \\
105\end{array}$ & $\begin{array}{c}12.00 \\
4.3 \\
1.41 \\
106\end{array}$ & 4 \\
\hline
\end{tabular}

leges; service load is 947 units. 7. Approximately. 8. Summer salaries not included. 9. 101/2 months. 10. Plus $\$ 5,836$ estimated nonsalaried service. 11. Includes $\$ 2,500$ estimated nonsalaried service. 12. Includes $\$ 15,815$ nonsalaried service. 13. Includes $\$ 15,459$ nonsalaried service. 14. Includes $\$ 8,000$ nonsalaried service. 15. Includes living quarters. 16. Plus $\$ 9,600$ nonsalaried service. 17. Includes $\$ 500$ nonsalaried service. 18. Includes $\$ 230$ nonsalaried service. 19. Number of libraries on which high, median and low are based.

\section{Statistics, I 955-I $95^{6}$ (Group III)}

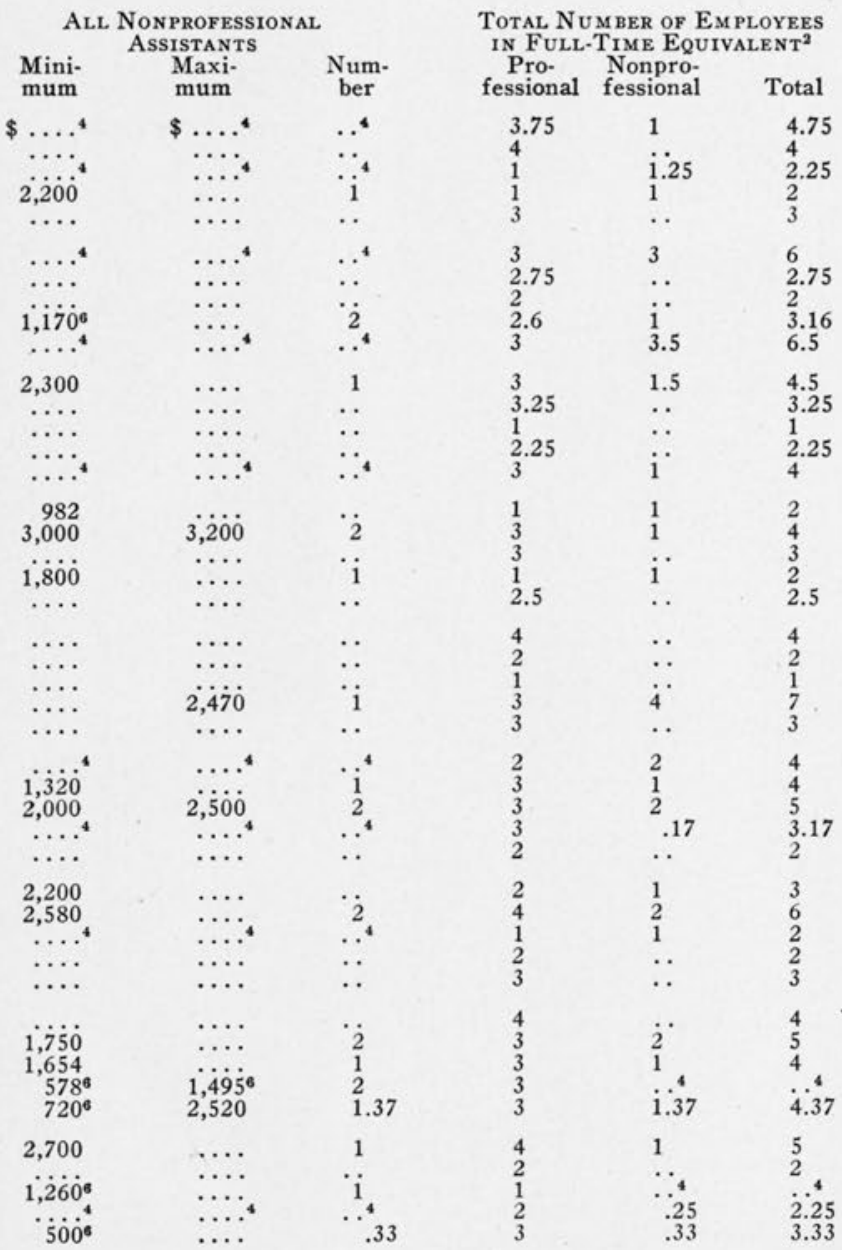

\begin{tabular}{|c|c|}
\hline $\begin{array}{c}\text { Working } \\
\text { Hours in } \\
\text { Fiscal } \\
\text { Year }^{3}\end{array}$ & $\begin{array}{c}\text { Hours of } \\
\text { Student } \\
\text { Assistance }\end{array}$ \\
\hline $\begin{array}{l}1,760^{4} \\
2,080 \\
1,760 \\
1,960\end{array}$ & $\begin{array}{l}1,326 \\
4,670 \\
1,827 \\
6,064 \\
4,481\end{array}$ \\
\hline $\begin{array}{l}1,8 \dot{4}^{4} \\
2,070 \\
1,568 \\
1,888\end{array}$ & $\begin{array}{l}4,472 \\
4,109 \\
4,249 \\
2,500\end{array}$ \\
\hline $\begin{array}{l}1,400 \\
1,650^{7} \\
1,368 \\
1,719 \\
1,861\end{array}$ & $\begin{array}{l}3,248 \\
720^{7} \\
2,268 \\
3,600 \\
1,306\end{array}$ \\
\hline $\begin{array}{l}1,516 \\
1,755 \\
2,888 \\
\ldots .4^{4}\end{array}$ & $\begin{array}{l}1,655 \\
6,080 \\
3,012 \\
1,060 \\
2,500\end{array}$ \\
\hline $\begin{array}{l}1,760 \\
\cdots\end{array}$ & $\begin{array}{l}6,000 \\
\cdots . .4\end{array}$ \\
\hline $\begin{array}{l}1,637 \\
2,024\end{array}$ & $\begin{array}{l}2,215 \\
3,610\end{array}$ \\
\hline $\begin{array}{l}1,833 \\
1,760 \\
1,868 \\
2,024\end{array}$ & $\begin{array}{r}4,538 \\
2,183 \\
906 \\
3,125 \\
6,368\end{array}$ \\
\hline $\begin{array}{l}2,000 \\
1,702 \\
2,322 \\
1,787 \\
1,665\end{array}$ & $\begin{array}{l}2,500 \\
1,762 \\
2,06 \dot{6} \\
2,873\end{array}$ \\
\hline $\begin{array}{l}1,326 \\
1,748 \\
1,800 \\
1,850^{4}\end{array}$ & $\begin{array}{r}7,000 \\
2,456 \\
960 \\
513 \\
3,537\end{array}$ \\
\hline $\begin{array}{l}1,610 \\
1,710 \\
2,025 \\
1,572 \\
1,518\end{array}$ & $\begin{array}{l}940^{17} \\
1,600 \\
3,000 \\
4,720 \\
5,301\end{array}$ \\
\hline
\end{tabular}




\section{College and University Library}

\begin{tabular}{|c|c|c|c|c|c|c|c|c|c|}
\hline & & $\begin{array}{r}\text { Associ } \\
\text { Assis } \\
\text { CHIEF L }\end{array}$ & $\begin{array}{l}\text { ATE OR } \\
\text { TANT } \\
\text { IBRARIAN }\end{array}$ & DEPART & MENT H & EADS & $\begin{array}{l}\text { ALL OT } \\
\text { SIONAL }\end{array}$ & $\begin{array}{l}\text { HER PRo } \\
\text { AsSISTA }\end{array}$ & $\begin{array}{l}\text { ES- } \\
\text { TS }\end{array}$ \\
\hline Library $^{1}$ & $\underset{\text { brarian }}{\mathrm{Li}-}$ & $\begin{array}{l}\text { Mini- } \\
\text { mum }\end{array}$ & $\begin{array}{l}\text { Maxi- } \\
\text { mum }\end{array}$ & $\begin{array}{l}\text { Mini- } \\
\text { mum }\end{array}$ & $\begin{array}{l}\text { Maxi- } \\
\text { mum }\end{array}$ & Num- & $\begin{array}{l}\text { Mini- } \\
\text { mum }\end{array}$ & $\begin{array}{l}\text { Maxi- } \\
\text { mum }\end{array}$ & $\begin{array}{l}\text { Num- } \\
\text { ber }\end{array}$ \\
\hline 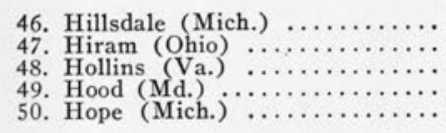 & $\begin{array}{l}3,900 \\
7,300 \\
4,300 \\
5,300\end{array}$ & $\begin{array}{l}\cdots \cdots \\
5,500 \\
3,000 \\
\cdots\end{array}$ & $\begin{array}{l}\cdots \cdots \\
\cdots \cdots \\
3,300 \\
\cdots \cdots\end{array}$ & $\begin{array}{l}\cdots, 300 \\
4,300\end{array}$ & $\begin{array}{l}\cdots \cdots \\
\cdots \cdots \\
4,900\end{array}$ & $\begin{array}{l}\because i^{4} \\
\ddot{2}\end{array}$ & $\begin{array}{l}\cdots \cdots \\
1,700^{\circ} \\
\cdots \cdots\end{array}$ & $\begin{array}{l}\cdots \cdots \\
\cdots \\
\cdots \\
\cdots\end{array}$ & $\begin{array}{l}\cdots \\
\cdots \\
\cdots\end{array}$ \\
\hline 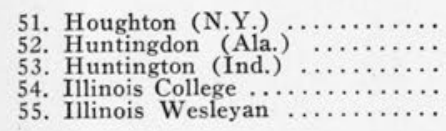 & $\begin{array}{l}3,000^{3} \\
4,980 \\
4,600^{4} \\
4,700\end{array}$ & $\begin{array}{l}\cdots \cdots \\
\cdots \cdots \\
\cdots \cdots\end{array}$ & $\begin{array}{l}3,129 \\
\cdots \cdots \\
\cdots \cdots\end{array}$ & $\begin{array}{l}1,600^{8} \\
\cdots \cdots \\
3,400^{4} \\
3,500\end{array}$ & $\begin{array}{l}\cdots \\
\cdots \\
\cdots \\
\cdots\end{array}$ & $\begin{array}{l}2 \\
\cdots \\
2 \\
2\end{array}$ & $\begin{array}{l}\cdots \cdots \\
\cdots \cdots \\
2,8 \overline{7}^{10}\end{array}$ & $\begin{array}{l}\cdots \cdots \\
\cdots \cdots \\
\cdots \cdots\end{array}$ & $\begin{array}{l}\because \\
\because \\
\therefore\end{array}$ \\
\hline 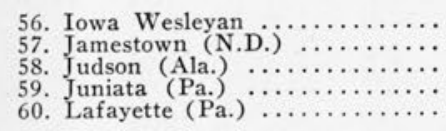 & $\begin{array}{l}3,300 \\
3,384 \\
4,100 \\
5,000\end{array}$ & $\begin{array}{r}3,2000 \\
\ldots \ldots\end{array}$ & $\begin{array}{l}\cdots \\
\cdots \cdots \\
\cdots\end{array}$ & $\begin{array}{l}\cdots \\
\cdots \cdots \\
\cdots \cdots\end{array}$ & $\begin{array}{l}\cdots \\
\cdots \\
\cdots \\
\cdots\end{array}$ & $\begin{array}{l}\cdots \\
\cdots \\
\cdots\end{array}$ & $\begin{array}{l}\cdots{ }^{4} \\
\cdots \cdots \\
3,500\end{array}$ & $\begin{array}{l}\cdots \cdots \\
\cdots \cdots \\
\cdots \\
\cdots\end{array}$ & $\begin{array}{l}\cdots \\
\cdots \\
\vdots \\
i\end{array}$ \\
\hline 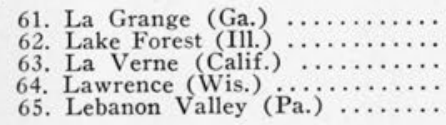 & $\begin{array}{l}3,850 \\
5,210 \\
4,140 \\
6,000 \\
\ldots \ldots\end{array}$ & $\begin{array}{l}\ldots \\
\ldots \ldots \\
4,200 \\
\ldots \ldots\end{array}$ & $\begin{array}{l}\cdots \cdots \\
\cdots \\
\cdots \\
\cdots\end{array}$ & $\begin{array}{l}\cdots \\
1,500^{11} \\
\cdots \cdots\end{array}$ & $\begin{array}{l}\cdots \\
\cdots \\
\cdots \\
\cdots\end{array}$ & $\begin{array}{l}\cdots \\
\because \\
\cdots\end{array}$ & $\begin{array}{l}3,600 \\
\cdots \cdots \\
\cdots \cdots\end{array}$ & $\begin{array}{l}3,800 \\
\ldots \ldots \\
\ldots \ldots\end{array}$ & $\begin{array}{c}1 \\
\cdots \\
\cdots\end{array}$ \\
\hline 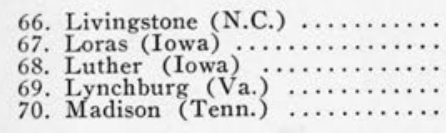 & $\begin{array}{l}3,841 \\
800 \\
6,300 \\
4,216 \\
1,506^{12}\end{array}$ & $\begin{array}{l}\cdots \\
\cdots \\
3,600 \\
1,800\end{array}$ & $\begin{array}{r}2,205 \\
\ldots \\
4,100 \\
\cdots\end{array}$ & $\begin{array}{l}\cdots \cdots \\
4,500 \\
\cdots \cdots\end{array}$ & $\begin{array}{l}\cdots \\
\cdots \cdots \\
\cdots\end{array}$ & $\begin{array}{l}\cdots \\
2 \\
\cdots\end{array}$ & $\begin{array}{l}\cdots \\
3,600 \\
\cdots \\
\cdots\end{array}$ & $\begin{array}{r}2,650 \\
4,000 \\
\cdots\end{array}$ & $\begin{array}{l}\cdots \\
2 \\
\cdots\end{array}$ \\
\hline 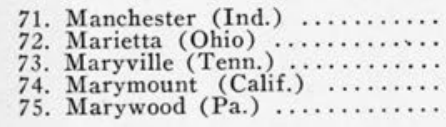 & $\begin{array}{r}4,700 \\
5,250 \\
\cdots \cdots 4 \\
4,300\end{array}$ & $\begin{array}{l}4,050 \\
3,100 \\
3,300 \\
3,800\end{array}$ & $\begin{array}{l}\cdots \\
\cdots \\
\cdots \\
\cdots\end{array}$ & $\begin{array}{l}\cdots \\
\cdots \\
\cdots \\
\cdots\end{array}$ & $\begin{array}{l}\cdots \\
\cdots \\
\cdots \\
\cdots\end{array}$ & $\begin{array}{l}\cdots \\
\because \\
\cdots\end{array}$ & $\begin{array}{l}\ldots \ldots \\
\ldots \ldots \\
3,600\end{array}$ & $\begin{array}{l}\cdots \\
\cdots \\
\cdots \\
\cdots\end{array}$ & $\begin{array}{l}\because 4 \\
i \\
i\end{array}$ \\
\hline 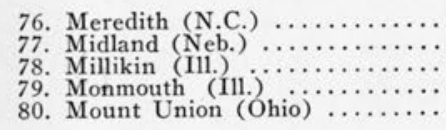 & $\begin{array}{l}3,050^{4} \\
4,200^{4} \\
\ldots{ }^{4}\end{array}$ & $\begin{array}{l}5,000^{4} \\
\ldots \ldots \\
\ldots \ldots\end{array}$ & $\begin{array}{l}\cdots{ }^{4} \\
\cdots \cdots \\
\cdots \cdots\end{array}$ & $\begin{array}{l}\cdots{ }^{4} \\
\cdots \\
\cdots \cdots \\
\cdots\end{array}$ & $\begin{array}{l}\cdots{ }^{4} \\
\cdots \\
\cdots \\
\cdots\end{array}$ & $\begin{array}{l}\cdots^{4} \\
\cdots \\
\cdots\end{array}$ & $\begin{array}{l}\ldots{ }^{4} \\
\cdots, 200^{4} \\
\ldots{ }^{4}\end{array}$ & $\begin{array}{l}3,600^{4} \\
3,300^{4}\end{array}$ & $\begin{array}{l}.^{4} \\
4^{5} \\
3^{4}\end{array}$ \\
\hline $\begin{array}{l}\text { 81. Muskingum (Ohio) } \ldots \ldots \ldots \ldots \\
\text { 82. Nebraska Wesleyan } \ldots \ldots \ldots \ldots \\
\text { 83. New Mexico Highlands } \ldots \ldots \ldots \\
\text { 84. Pacific Union (Calif.) } \ldots \ldots \ldots \ldots \\
\text { 85. Paine (Ga.) } \ldots \ldots \ldots \ldots \ldots \ldots \ldots\end{array}$ & $\begin{array}{l}4,000 \\
4,600 \\
\cdots \cdots{ }^{13} \\
\cdots, 450\end{array}$ & $\begin{array}{l}2,700 \\
5,000 \\
\ldots \\
\cdots\end{array}$ & $\begin{array}{l}\cdots \\
\cdots, 200 \\
\cdots \cdots\end{array}$ & $\begin{array}{l}\cdots \\
4,200 \\
\ldots \ldots\end{array}$ & $\begin{array}{l}\cdots \\
5,400 \\
\cdots \cdots\end{array}$ & $\begin{array}{l}\cdots \\
\because \\
\cdots\end{array}$ & $\begin{array}{l}3,000 \\
\cdots \\
\cdots \\
\cdots\end{array}$ & $\begin{array}{l}3,600 \\
\cdots \cdots \\
\cdots \cdots \\
\cdots\end{array}$ & $\begin{array}{l}2 \\
\cdots \\
\cdots\end{array}$ \\
\hline 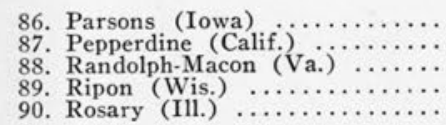 & $\begin{array}{l}4,200 \\
3,900 \\
4,600^{4} \\
\ldots \ldots 4\end{array}$ & $\begin{array}{l}\cdots \\
\cdots \\
\cdots \\
\cdots\end{array}$ & $\begin{array}{l}1,116^{6} \\
\cdots \cdots \\
\cdots \cdots\end{array}$ & $\begin{array}{l}3,300 \\
\cdots \\
\cdots\end{array}$ & $\begin{array}{r}3,600 \\
\cdots \\
\cdots\end{array}$ & $\begin{array}{l}\ddot{2} \\
\cdots \\
\cdots\end{array}$ & $\begin{array}{l}1,281^{6} \\
3,255^{4} \\
\cdots \cdots\end{array}$ & $\begin{array}{l}\cdots \\
4,300 \\
\ldots \\
\ldots\end{array}$ & $\begin{array}{l}1 \\
3 \\
\cdots 4\end{array}$ \\
\hline $\begin{array}{l}\text { 91. Saint Joseph's Coll. (Ind.) } \ldots \ldots \\
\text { 92. Savannah (Ga.) } \ldots \ldots \ldots \ldots \ldots \ldots \\
\text { 93. Scripps (Calif.) } \ldots \ldots \ldots \ldots \ldots \ldots \\
\text { 94. Seton Hill (Pa.) } \ldots \ldots \ldots \ldots \ldots \\
\text { 95. Simpson (Iowa) } \ldots \ldots \ldots \ldots \ldots\end{array}$ & $\begin{array}{l}8,000 \\
5,100 \\
4,000 \\
4,300\end{array}$ & $\begin{array}{l}5,600 \\
4,520 \\
3,000 \\
3,700^{14}\end{array}$ & $\begin{array}{r}7,000 \\
4,600 \\
3,400 \\
\cdots \cdots \\
\cdots\end{array}$ & $\begin{array}{l}\cdots \\
\cdots \cdots \\
\cdots \cdots\end{array}$ & $\begin{array}{l}\cdots \\
\cdots \cdots \\
\cdots \cdots\end{array}$ & $\begin{array}{l}\cdots \\
\therefore \\
\therefore\end{array}$ & $\begin{array}{l}\cdots \\
\cdots \\
1,000^{\circ} \\
\cdots\end{array}$ & $\begin{array}{l}\cdots \\
\cdots \cdots \\
\cdots \cdots\end{array}$ & $\ldots$ \\
\hline 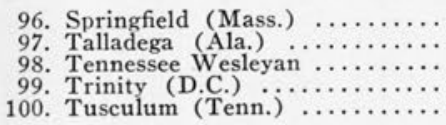 & $\begin{array}{l}3, \ldots 0^{4} \\
3,900 \\
3,300^{16}\end{array}$ & $\begin{array}{l}3,220^{15} \\
1,500 \\
\ldots \ldots \\
\ldots \ldots\end{array}$ & $\begin{array}{l}\cdots \\
\cdots \\
\cdots \\
\cdots\end{array}$ & $\begin{array}{l}\cdots \cdots \\
\cdots \\
\cdots \\
\cdots\end{array}$ & $\begin{array}{l}\cdots \\
\cdots \\
\cdots \\
\cdots\end{array}$ & $\begin{array}{l}\cdots \\
\because \\
\cdots\end{array}$ & $\begin{array}{l}\ldots \ldots \\
\cdots \cdots \\
3,500 \\
\ldots \ldots\end{array}$ & $\begin{array}{l}\ldots{ }^{4} \\
\ldots \\
\cdots \\
\cdots\end{array}$ & $\begin{array}{l}\cdots \\
\cdots \\
i\end{array}$ \\
\hline 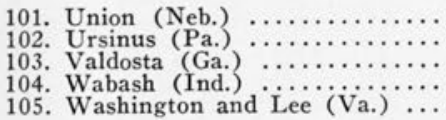 & $\begin{array}{l}2,503 \\
5,200 \\
4,700 \\
\ldots \ldots \\
\ldots \ldots\end{array}$ & $\begin{array}{l}2,494 \\
4,200 \\
4,000 \\
\ldots \ldots\end{array}$ & $\begin{array}{l}\cdots \\
\cdots \cdots \\
\cdots \cdots\end{array}$ & $\begin{array}{l}\cdots \\
\cdots \cdots \\
3,800\end{array}$ & $\cdots \cdots$ & $\begin{array}{l}\cdots \\
\because \\
3\end{array}$ & $\begin{array}{l}\cdots \\
\cdots \\
\cdots \\
\cdots\end{array}$ & $\begin{array}{l}\cdots \\
\cdots \\
\cdots \\
\cdots\end{array}$ & $\begin{array}{l}\cdots \\
\cdots \\
\cdots\end{array}$ \\
\hline 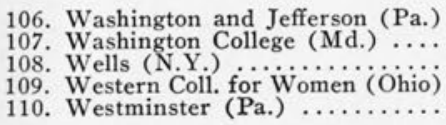 & $\begin{array}{l}5,500 \\
5,500 \\
5,100 \\
4,500 \\
4,800\end{array}$ & $\begin{array}{l}\dot{4,500} \\
\cdots \\
3,600\end{array}$ & $\begin{array}{l}\cdots \\
\cdots \\
3, \ddot{9} 0 \dot{0}\end{array}$ & $\begin{array}{l}\cdots \\
\cdots \cdots \\
\cdots \cdots\end{array}$ & $\begin{array}{l}\cdots \\
\cdots \cdots \\
\cdots\end{array}$ & $\begin{array}{l}\cdots \\
\cdots \\
\cdots\end{array}$ & $\begin{array}{l}3,000 \\
1,200^{6} \\
3,400 \\
\cdots \cdots\end{array}$ & $\begin{array}{c}\cdots \\
\cdots \\
\cdots \\
\cdots \\
\cdots\end{array}$ & $\begin{array}{l}{ }^{.33} \\
\cdots^{4}\end{array}$ \\
\hline $\begin{array}{l}\text { 111. Whittier (Calif.) } \ldots \ldots \ldots \ldots \ldots \\
\text { 112. Wilberforce (Ohio) } \ldots \ldots \ldots \ldots \ldots \\
\text { 113. Willamette (Ore.) } \ldots \ldots \ldots \ldots \ldots\end{array}$ & $\begin{array}{l}5,688 \\
3,600 \\
\ldots \ldots\end{array}$ & $\cdots \cdots$ & $\begin{array}{l}3,800 \\
\cdots \cdots\end{array}$ & $\begin{array}{l}\ldots \\
\ldots \ldots \\
\ldots\end{array}$ & $\begin{array}{l}\cdots \\
\cdots{ }^{4}\end{array}$ & $\because$ & $\begin{array}{l}\cdots \\
\cdots \cdot{ }^{4}\end{array}$ & $\begin{array}{l}\cdots \\
\cdots \cdot{ }^{4}\end{array}$ & $\because$ \\
\hline 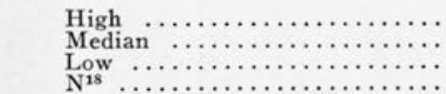 & $\begin{array}{r}8,000 \\
4,700 \\
800 \\
86\end{array}$ & $\begin{array}{r}5,600 \\
3,700 \\
1,500 \\
44\end{array}$ & $\begin{array}{r}7,000 \\
3,800 \\
2,205 \\
17\end{array}$ & $\begin{array}{r}4,571 \\
3,533 \\
2,700 \\
13\end{array}$ & $\begin{array}{r}5,968 \\
3,700 \\
3,350 \\
8\end{array}$ & $\begin{array}{c}3 \\
2 \\
14^{-5}\end{array}$ & $\begin{array}{r}3,780 \\
3,500 \\
1,800 \\
20\end{array}$ & $\begin{array}{l}4,300 \\
3,800 \\
2,650 \\
9\end{array}$ & $\begin{array}{c}3 \\
1 \\
24 \\
.33\end{array}$ \\
\hline
\end{tabular}

1. Central library and all agencies. 2. Excludes student assistants and building maintenance staff. 3. Excludes vacations and legal holidays. 4. Not reported or not available. 5. Plus extra pay for summer. 6. Part-time. 7.Ap. proximately. 8. Nine months. 9. Includes academic teaching. 10. Three-fourth's time. 11. Library consultant 
Salary Statistics, $1955^{-5} 6$ (Group III, Cont.)

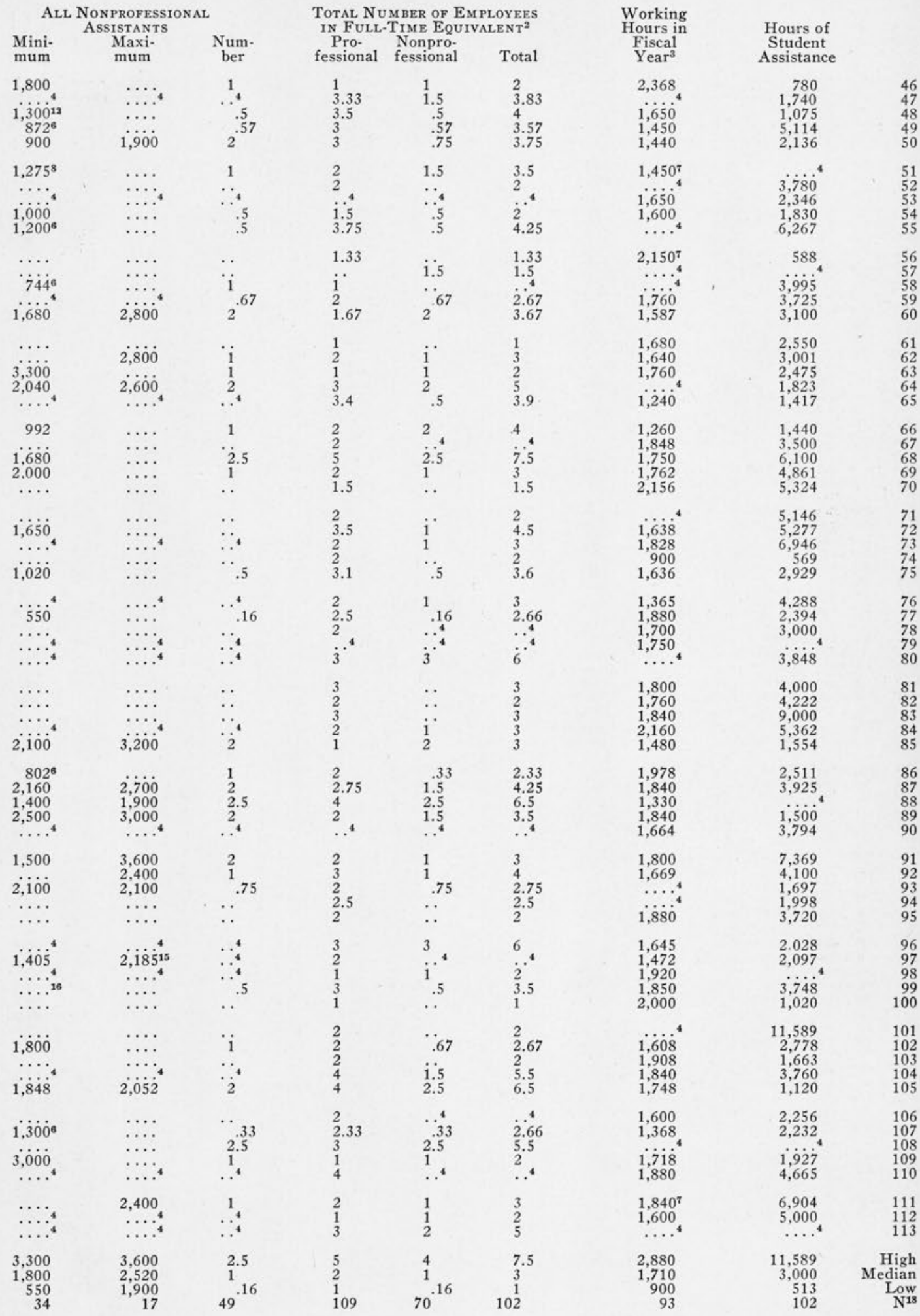

one-fourth time. 12. Half-time. 13. $\$ 5.500-\$ 8,000$ range. 14. Ten months. 15. Includes living quarters. 16 . Nonsalaried service. 17. Plus 2,366 hours paid out of other funds. 18. Number of libraries on which high, median, and low are based. 
Library $^{1}$

1. Alabama, Troy, STC

2. Arizona, Flagstaff, STC

3. California, Arcata, Humboldt $\mathrm{S}^{\mathrm{S}} \ldots \ldots \ldots \ldots$

5. California, Chico, $\mathrm{SC}$. $\ldots \ldots \ldots \ldots \ldots \ldots \ldots \ldots$

6. California, Long Beach

7. California, Sacramento, SC

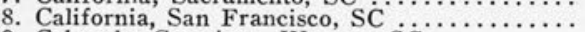

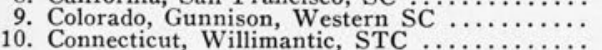

11. Georgia, Collegeboro, TC

12. Illinois, Charleston Eastern $\mathrm{s}_{\mathrm{C}} \ldots \ldots \ldots \ldots \ldots$

13. Illinois, DeKalb, Northern SC.$\ldots \ldots \ldots \ldots$.

14. Indiana, Muncie, Ball STC $\ldots \ldots \ldots \ldots \ldots \ldots$

16. Iowa, Cedar Falls, STC $\ldots \ldots \ldots \ldots \ldots \ldots$

17. Kansas, Emporia, STC $\ldots \ldots \ldots \ldots \ldots \ldots \ldots \ldots$

18. Kansas, Pittsburg, STC $\ldots \ldots \ldots \ldots \ldots$

20. Kentucky, Morehead, SC ..................

21. Kentucky, Murray, SC

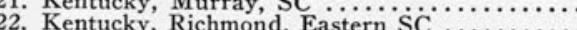

23. Louisiana, Lafayette, Southwestern La. Inst.

24. Louisiana, Natchitoches, Northwestern SC ...

25. Maryland, Frostburg, STC ...............

26. Maryland, Salisbury, STC $\ldots \ldots \ldots \ldots \ldots \ldots$

27. Maryland, Towson, STC

28. Massachusetts, Fitchburg, STC

9. Massachusetts, Framingham, STC ${ }^{2} \ldots \ldots \ldots$

30. Massachusetts, North Adams, STC $\ldots \ldots \ldots$.

31. Michigan, Kalamazoo, Western Mich. Coll. ${ }^{10}$ 32. Michigan, Mt. Pleasant, Central Michigan Coll 33. Michigan, Marquette, Northern Mich. Coll.

34. Michigan, Ypsilanti, Eastern Mich. Coll. ....

35. Minnesota, Bemidji, STC ..................

36. Minnesota, Mankato, STC

37. Minnesota, Moorhead, STC ...................

38. Minnesota, St. Cloud, STC $\ldots . . \ldots \ldots \ldots \ldots \ldots . . . . .$.

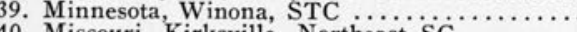

40. Missouri, Kirksville, Northeast SC ...........

41. Missouri, Springfield, Southwest SC ........

42. Nebraska, Chadron, STC $\ldots \ldots \ldots \ldots \ldots \ldots . . . .$.

43. Nebraska, Kearney, STC $\ldots \ldots \ldots \ldots \ldots \ldots \ldots \ldots \ldots \ldots .$.

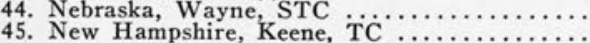

46. New Jersey, Glassboro, STC $\ldots \ldots \ldots \ldots \ldots$

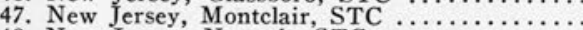

48. New Jersey, Newark, STC $\ldots \ldots \ldots \ldots \ldots \ldots$

50. New Jersey, Trenton, STC $\ldots \ldots \ldots \ldots \ldots \ldots \ldots$

51. New York, Albany, STC

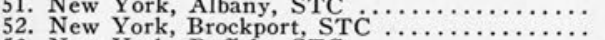

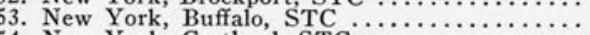

54. New York, Cortland, STC $\ldots \ldots \ldots \ldots \ldots \ldots$

56. New York, Oswego, STC

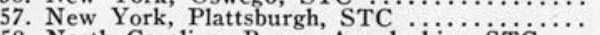

58. North Carolina, Boone, Appalachian STC ...

59. North Carolina, Elizabeth City, STC ......

0. North Carolina, Fayetteville, STC .........

61. North Carolina, Greenville, East Carolina Coll. 62. North Carolina, Winston-Salem, STC ...... 63. North Dakota, Dickinson, STC $\ldots \ldots \ldots \ldots$ 64. North Dakota, Valley City, STC $\ldots \ldots \ldots \ldots$
Total

\begin{tabular}{|c|c|c|c|c|c|c|c|}
\hline $\begin{array}{l}\text { Fiscal } \\
\text { Year } \\
\text { Ending }\end{array}$ & $\begin{array}{l}\text { Under- } \\
\text { grad- } \\
\text { uate }\end{array}$ & $\begin{array}{l}\text { Total } \\
\text { Grad- } \\
\text { uate }\end{array}$ & $\begin{array}{l}\text { Total } \\
\text { Exten- } \\
\text { sion }\end{array}$ & $\begin{array}{l}\text { Book } \\
\text { Stock }\end{array}$ & $\begin{array}{l}\text { Volumes } \\
\text { Added }\end{array}$ & News- & $\begin{array}{l}\text { Peri- } \\
\text { odicals }\end{array}$ \\
\hline $\begin{array}{r}30 \mathrm{~S} 56 \\
30 \mathrm{Je} 56 \\
30 \mathrm{Je} 56 \\
30 \mathrm{Je} 56 \\
30 \mathrm{Je} 56\end{array}$ & $\begin{array}{r}1,348 \\
934 \\
1,075 \\
2,097 \\
4,129\end{array}$ & $\begin{array}{r}* 45 \\
33 \\
101 \\
758\end{array}$ & $\begin{array}{r}277 \\
68 \\
175 \\
316 \\
1,047\end{array}$ & $\begin{array}{r}42,686 \\
53,073 \\
56,432 \\
72,526 \\
112,141\end{array}$ & $\begin{array}{l}2,093 \\
3,285 \\
4,211 \\
5,111 \\
8,390\end{array}$ & $\begin{array}{r}13 \\
7 \\
25 \\
21 \\
17\end{array}$ & $\begin{array}{r}310 \\
340 \\
605 \\
657 \\
1,051\end{array}$ \\
\hline $\begin{array}{r}\text { 30Je56 } \\
\text { 30Je56 } \\
\text { 30Je56 } \\
31 \mathrm{Ag} 56 \\
\text { 30Je56 }\end{array}$ & $\begin{array}{r}3,792 \\
2,657 \\
6,776 \\
664 \\
487\end{array}$ & $\begin{array}{r}1,553 \\
1,457 \\
1,227 \\
168 \\
\ldots .\end{array}$ & $\begin{array}{c}\ddot{426} \\
1,064 \\
31 \dot{9}^{6}\end{array}$ & $\begin{array}{r}58,8 \\
65,4 \\
114,1 \\
60,6 \\
19,9\end{array}$ & $\begin{array}{r}10,285 \\
13,041 \\
9,593 \\
2,251 \\
1,153\end{array}$ & $\begin{array}{r}13 \\
11 \\
22 \\
23 \\
5\end{array}$ & $\begin{array}{r}580 \\
888 \\
1,003 \\
432 \\
225\end{array}$ \\
\hline $\begin{array}{l}30 \mathrm{Je} 56 \\
30 \mathrm{Je} 56 \\
30 \mathrm{Je} 56 \\
30 \mathrm{Je} 56 \\
30 \mathrm{Je} 56\end{array}$ & $\begin{array}{r}763 \\
1,893 \\
2,899 \\
3,909 \\
2,564\end{array}$ & $\begin{array}{r}\cdots 22 \\
361 \\
432 \\
287\end{array}$ & $\begin{array}{l}213 \\
395 \\
227 \\
397\end{array}$ & $\begin{array}{r}53,400 \\
93,040 \\
106,664\end{array}$ & $\begin{array}{l}2, \\
3, \\
5, \\
8, \\
4,\end{array}$ & $\begin{array}{l}16 \\
27 \\
16 \\
15 \\
22\end{array}$ & $\begin{array}{l}701 \\
792 \\
658 \\
520\end{array}$ \\
\hline $\begin{array}{l}30 \mathrm{Je} 56 \\
30 \mathrm{Je} 56 \\
30 \mathrm{Je} 56 \\
30 \mathrm{Je} 56 \\
30 \mathrm{Je} 56\end{array}$ & $\begin{array}{r}2,801 \\
1,994 \\
2,244 \\
1,722 \\
998\end{array}$ & $\begin{array}{r}122 \\
143 \\
70 \\
16 \\
62\end{array}$ & $\begin{array}{l}365 \\
589 \\
988 \\
156 \\
661\end{array}$ & $\begin{array}{r}117,3 \\
101,6 \\
102,5 \\
51,4\end{array}$ & $\begin{array}{l}9 \\
35^{7} \\
9 \\
2 \\
5\end{array}$ & $\begin{array}{l}26 \\
12 \\
30 \\
11 \\
29\end{array}$ & $\begin{array}{l}765 \\
561 \\
845 \\
400 \\
410\end{array}$ \\
\hline $\begin{array}{l}30 \mathrm{Je} 56 \\
30 \mathrm{Je} 56 \\
30 \mathrm{Je} 56 \\
30 \mathrm{Je} 56 \\
30 \mathrm{Je} 56\end{array}$ & $\begin{array}{r}1,845 \\
2,078 \\
3,759 \\
2,004 \\
621\end{array}$ & $\begin{array}{r}52 \\
114 \\
\because 116 \\
0\end{array}$ & $\begin{array}{r}563 \\
\cdots \cdots \\
\cdots \\
80 \\
8\end{array}$ & $\begin{array}{r}56,1 \\
95,2 \\
113,8 \\
79,0 \\
27,3\end{array}$ & $\begin{array}{l}2, \\
6, \\
6, \\
1,\end{array}$ & $\begin{array}{l}1 \\
2 \\
2 \\
2 \\
1\end{array}$ & $\begin{array}{l}465 \\
318 \\
666 \\
601 \\
248\end{array}$ \\
\hline $\begin{array}{l}30 \mathrm{Je} 56 \\
30 \mathrm{Je} 56 \\
30 \mathrm{Je} 56 \\
30 \mathrm{Je} 56 \\
30 \mathrm{Je} 56\end{array}$ & $\begin{array}{r}428 \\
1,209 \\
530 \\
603 \\
172\end{array}$ & $\begin{array}{l}\cdots \\
207 \\
\ldots \\
\ldots\end{array}$ & $\begin{array}{l}\cdots \\
\cdots \\
445^{\circ} \\
\cdots \\
76\end{array}$ & $\begin{array}{l}26,286 \\
49,664 \\
33,000 \\
22,740 \\
13,735\end{array}$ & $\begin{array}{r}3,014 \\
1,500 \\
375 \\
542\end{array}$ & $\begin{array}{r}7 \\
8 \\
5 \\
\hdashline\end{array}$ & $\begin{array}{l}167 \\
257 \\
300 \\
201 \\
128\end{array}$ \\
\hline $\begin{array}{l}30 \mathrm{Je} 56 \\
30 \mathrm{Je} 56 \\
30 \mathrm{Je} 56 \\
30 \mathrm{Je} 56 \\
30 \mathrm{Je} 56\end{array}$ & $\begin{array}{r}5,285 \\
2,856 \\
888 \\
3,050 \\
706\end{array}$ & $\begin{array}{r}465 \\
135 \\
3350 \\
\ldots \ldots\end{array}$ & $\begin{array}{r}1,577 \\
1,481 \\
1,200 \\
427\end{array}$ & $\begin{array}{r}84,195 \\
55,570 \\
116,830 \\
34,979\end{array}$ & $\begin{array}{l}3,6 \\
1,2 \\
5,( \\
1,5\end{array}$ & $\begin{array}{r}28 \\
12 \\
18 \\
12 \\
9\end{array}$ & $\begin{array}{r}1,199 \\
545 \\
347 \\
496 \\
250\end{array}$ \\
\hline $\begin{array}{l}\text { 30Je56 } \\
\text { 30Je56 } \\
\text { 30Je56 } \\
\text { 30Je56 } \\
\text { 30Je56 }\end{array}$ & $\begin{array}{r}2,534 \\
844 \\
1,925 \\
748 \\
1,509\end{array}$ & $\begin{array}{r}109 \\
\cdots 22 \\
54 \\
108\end{array}$ & $\begin{array}{l}677 \\
344 \\
321 \\
246 \\
305\end{array}$ & $\begin{array}{r}55,500 \\
37,121 \\
88,896 \\
42,735 \\
123,870\end{array}$ & & $\begin{array}{r}7 \\
12 \\
13 \\
13 \\
26\end{array}$ & $\begin{array}{l}411 \\
368 \\
502 \\
304 \\
531\end{array}$ \\
\hline $\begin{array}{l}\text { 30Je56 } \\
\text { 30Je56 } \\
\text { 30Je56 } \\
\text { 30Je56 } \\
\text { 30Je56 }\end{array}$ & $\begin{array}{r}2,419 \\
454 \\
1,115 \\
896 \\
621\end{array}$ & $\begin{array}{l}146^{11} \\
\cdots \\
\cdots \\
{ }_{14}\end{array}$ & $\begin{array}{r}599 \\
287 \\
404 \\
72\end{array}$ & $\begin{array}{l}54,0 \\
44,8 \\
50,2 \\
25,8\end{array}$ & $\begin{array}{l}2,2 \\
1,2 \\
1,8 \\
1,5\end{array}$ & $\begin{array}{r}9 \\
46 \\
43 \\
8 \\
10\end{array}$ & $\begin{array}{l}331 \\
226 \\
241 \\
305 \\
203\end{array}$ \\
\hline $\begin{array}{l}30 \mathrm{Je} 56 \\
30 \mathrm{Je} 56 \\
30 \mathrm{Je} 56 \\
30 \mathrm{Je} 56 \\
30 \mathrm{Je} 56\end{array}$ & $\begin{array}{r}518 \\
1,218 \\
1,042 \\
1,188 \\
940\end{array}$ & $\begin{array}{l}323 \\
565^{13} \\
807 \\
69 \\
24^{11}\end{array}$ & $\begin{array}{r}677 \\
24 \\
391 \\
85 \\
909\end{array}$ & $\begin{array}{l}40,6 \\
71,7 \\
34,0 \\
24,3 \\
78,0\end{array}$ & $\begin{array}{r}2,9 \\
9 \\
1,4 \\
4,7\end{array}$ & $\begin{array}{l}7 \\
8 \\
5 \\
7 \\
8\end{array}$ & $\begin{array}{l}173 \\
350 \\
222 \\
265 \\
292\end{array}$ \\
\hline $\begin{array}{l}31 \mathrm{Mr} 56 \\
31 \mathrm{Mr} 56 \\
31 \mathrm{Mr} 56 \\
31 \mathrm{Mr} 56 \\
31 \mathrm{Mr} 56\end{array}$ & $\begin{array}{l}1,795^{14} \\
1,065 \\
2,514 \\
1,919 \\
937\end{array}$ & $\begin{array}{l}153^{14} \\
196 \\
375 \\
77 \\
51\end{array}$ & $\begin{array}{l}756^{14} \\
290 \\
670 \\
398\end{array}$ & $\begin{array}{l}55,0 \\
41,8 \\
63,0 \\
49,4 \\
43,1\end{array}$ & $\begin{array}{l}2, \\
2, \\
4, \\
3, \\
3,\end{array}$ & $\begin{array}{l}7 \\
5 \\
6 \\
6 \\
5\end{array}$ & $\begin{array}{l}386 \\
283 \\
456 \\
524 \\
287\end{array}$ \\
\hline $\begin{array}{r}31 \mathrm{Mr} 56 \\
31 \mathrm{Mr} 56 \\
30 \mathrm{Je} 56 \\
30 \mathrm{Je} 56 \\
30 \mathrm{Je} 56\end{array}$ & $\begin{array}{r}1,461 \\
961 \\
1,618 \\
379\end{array}$ & $\begin{array}{l}174 \\
113 \\
193\end{array}$ & $\begin{array}{r}157 \\
121 \\
27\end{array}$ & $\begin{array}{l}46,61 \\
66,78 \\
27,56 \\
37,01\end{array}$ & $\begin{array}{r}4,037 \\
2,653 \\
3,581 \\
510 \\
1,508\end{array}$ & $\begin{array}{r}13 \\
6 \\
28 \\
11 \\
13\end{array}$ & $\begin{array}{l}410 \\
411 \\
205 \\
253\end{array}$ \\
\hline $\begin{array}{l}\text { 30Je56 } \\
\text { 30Je56 } \\
\text { 30Je56 } \\
\text { 30Je56 } \\
\text { 30Je56 }\end{array}$ & $\begin{array}{r}2,774 \\
829 \\
409 \\
544 \\
705\end{array}$ & $\begin{array}{l}15 \\
\cdots \\
\cdots\end{array}$ & $\begin{array}{r}757 \\
\cdots 92 \\
92 \\
14 \dot{1}\end{array}$ & $\begin{array}{c}103,203 \\
\dddot{23}, 740^{6} \\
35,367 \\
39,821\end{array}$ & $\begin{array}{r}5,903 \\
747 \\
759 \\
547 \\
1,260\end{array}$ & $\begin{array}{r}13 \\
19 \\
25 \\
5 \\
23\end{array}$ & $\begin{array}{l}467 \\
346 \\
184 \\
139 \\
324\end{array}$ \\
\hline
\end{tabular}




\begin{tabular}{|c|c|c|c|c|c|c|c|c|c|c|}
\hline $\begin{array}{c}\text { Staff } \\
\text { Salaries }\end{array}$ & $\begin{array}{l}\text { Student } \\
\text { Service }\end{array}$ & $\begin{array}{l}\text { Total } \\
\text { Staff } \\
\text { Salaries } \\
\text { and } \\
\text { Student } \\
\text { Service }\end{array}$ & $\begin{array}{c}\text { Books } \\
\text { and } \\
\text { Related } \\
\text { Materials }\end{array}$ & Binding & $\begin{array}{l}\text { Total } \\
\text { for } \\
\text { Books } \\
\text { and } \\
\text { Binding }\end{array}$ & $\begin{array}{l}\text { Other } \\
\text { Operating } \\
\text { Expendi- }_{\text {tures }^{3}}\end{array}$ & $\begin{array}{c}\text { Total } \\
\text { Operat- } \\
\text { ing Ex- } \\
\text { pendi- } \\
\text { tures }\end{array}$ & $\begin{array}{l}\text { Per Stu- } \\
\text { dent Op- } \\
\text { erating } \\
\text { Expendi- } \\
\text { tures }\end{array}$ & $\begin{array}{l}\text { Total Edu- } \\
\text { cational } \\
\text { and Gen- } \\
\text { eral Insti- } \\
\text { tutional } \\
\text { Expendi- } \\
\text { tures }\end{array}$ & $\begin{array}{l}\text { Ratio of } \\
\text { Library } \\
\text { Expendi- } \\
\text { tures to } \\
\text { Total } \\
\text { (Per Cent) }\end{array}$ \\
\hline $\begin{array}{r}\$ 11,761 \\
12,500 \\
54,126 \\
55,714 \\
110,350\end{array}$ & $\begin{array}{r}\$ 4,267 \\
1,199 \\
4,800 \\
8,700 \\
14,893\end{array}$ & $\begin{array}{r}\$ 16,028 \\
13,699 \\
58,926 \\
64,414 \\
125,243\end{array}$ & $\begin{array}{r}\$ 9,630 \\
13,263 \\
18,260 \\
18,664 \\
29,999\end{array}$ & $\begin{array}{r}\$ 1,734 \\
1,470 \\
1,950 \\
2,433 \\
3,206\end{array}$ & $\begin{array}{r}\$ 10,364 \\
14,733 \\
20,210 \\
21,097 \\
33,205\end{array}$ & $\begin{array}{r}\$ 10,743 \\
875 \\
2,759 \\
2,242 \\
3,801\end{array}$ & $\begin{array}{r}\$ 27,135 \\
29,307 \\
81,895 \\
87,753 \\
162,249\end{array}$ & $\begin{array}{c}\$ 25.33^{5} \\
29.94^{5} \\
63.83 \\
35.00 \\
33.20^{5}\end{array}$ & $\begin{array}{r}597,794 \\
768,388 \\
1,231,659 \\
1,528,710 \\
3,332,468\end{array}$ & $\begin{array}{l}4.54 \\
3.8 \\
6.6 \\
5.7 \\
4.86\end{array}$ \\
\hline $\begin{array}{r}98,218 \\
79,503 \\
113,153 \\
11,020 \\
14,120\end{array}$ & $\begin{array}{r}15,978 \\
7,950 \\
21,756 \\
3,926 \\
900\end{array}$ & $\begin{array}{r}114,196 \\
87,453 \\
154,909 \\
14,946 \\
15,020\end{array}$ & $\begin{array}{r}42,089 \\
38,198 \\
43,531 \\
6,594 \\
3,900\end{array}$ & $\begin{array}{r}7,167 \\
3,957 \\
4,949 \\
519 \\
350\end{array}$ & $\begin{array}{r}49,256 \\
42,155 \\
48,480 \\
7,113 \\
4,250\end{array}$ & $\begin{array}{r}2,833 \\
6,524 \\
4,406 \\
1,053 \\
250\end{array}$ & $\begin{array}{r}166,285 \\
136,132 \\
207,795 \\
23,112 \\
19,520\end{array}$ & $\begin{array}{l}31.11 \\
29.98 \\
22.90 \\
27.68 \\
40.08^{5}\end{array}$ & $\begin{array}{c}2,119,380 \\
1,654,229 \\
4,019,807 \\
\cdots, 34.96 \\
443,962\end{array}$ & $\begin{array}{l}7.8 \\
8.2 \\
5.17 \\
.9 \\
4.4\end{array}$ \\
\hline $\begin{array}{r}18,154 \\
53,840 \\
67,184 \\
134,096 \\
48,335\end{array}$ & $\begin{array}{r}2,983 \\
10,002 \\
13,071 \\
34,907 \\
5,600\end{array}$ & $\begin{array}{r}21,137 \\
63,842 \\
80,255 \\
169,003 \\
53,935\end{array}$ & $\begin{array}{r}6,027 \\
15,904 \\
24,982 \\
33,050 \\
17,000\end{array}$ & $\begin{array}{l}1,077 \\
1,885 \\
3,210 \\
4,046 \\
4,000\end{array}$ & $\begin{array}{r}7,104 \\
17,789 \\
28,192 \\
37,096 \\
21,000\end{array}$ & $\begin{array}{r}365 \\
2,554 \\
4,808 \\
10,872 \\
3,625\end{array}$ & $\begin{array}{r}28,607 \\
84,185 \\
113,255 \\
216,971 \\
78,560\end{array}$ & $\begin{array}{l}37.49 \\
44.00^{5} \\
30.98 \\
49.98^{5} \\
27.55\end{array}$ & $\begin{array}{r}523,367 \\
1,827,857 \\
2,502,801 \\
2,865,332 \\
2,241,299\end{array}$ & $\begin{array}{l}5.47 \\
4.6 \\
4.5 \\
7.57 \\
3.5\end{array}$ \\
\hline $\begin{array}{l}57,935 \\
38,715 \\
32,903 \\
27,338 \\
17,562\end{array}$ & $\begin{array}{r}11,394 \\
13,487 \\
11,158 \\
5,269 \\
1,767\end{array}$ & $\begin{array}{l}69,329 \\
52,202 \\
44,061 \\
32,607 \\
19,329\end{array}$ & $\begin{array}{r}32,220 \\
32,445 \\
12,798 \\
9,439 \\
9,739\end{array}$ & $\begin{array}{l}9,234 \\
1,116 \\
2,187 \\
750 \\
1,405\end{array}$ & $\begin{array}{l}41,454 \\
33,561 \\
14,985 \\
10,189 \\
11,144\end{array}$ & $\begin{array}{l}6,052 \\
3,777 \\
2,924 \\
1,265 \\
1,036\end{array}$ & $\begin{array}{r}116,835 \\
89,540 \\
61,970 \\
43,961 \\
31,509\end{array}$ & $\begin{array}{l}39.97^{5} \\
32.84^{8} \\
26.82^{5} \\
25.55^{5} \\
29.72^{5}\end{array}$ & $\begin{array}{r}3,111,003 \\
2,038,371 \\
1,777,563 \\
964,698 \\
627,541\end{array}$ & $\begin{array}{l}3.76 \\
4.3 \\
3,6 \\
4.55 \\
5\end{array}$ \\
\hline $\begin{array}{l}14,600 \\
18,905 \\
72,334 \\
46,062 \\
15,955\end{array}$ & $\begin{array}{r}3,200 \\
4,181 \\
15,863 \\
8,431 \\
832\end{array}$ & $\begin{array}{l}17,800 \\
23,086 \\
88,196 \\
54,493 \\
16,787\end{array}$ & $\begin{array}{r}11,000 \\
9,989 \\
29,508 \\
36,981 \\
6,134\end{array}$ & $\begin{array}{r}1,800 \\
400 \\
2,297 \\
2,685 \\
592\end{array}$ & $\begin{array}{r}12,800 \\
10,389 \\
31,805 \\
39,666 \\
6,726\end{array}$ & $\begin{array}{r}1,275 \\
678 \\
5,745 \\
4,699 \\
800\end{array}$ & $\begin{array}{r}31,875 \\
34,153 \\
125,747 \\
98,858 \\
24,313\end{array}$ & $\begin{array}{l}12.96 \\
15.58 \\
33.45 \\
44.94 \\
38.65\end{array}$ & $\begin{array}{r}1,021,320 \\
2,464,989 \\
1,915,923 \\
487,509\end{array}$ & $\begin{array}{l}3.6 \\
3.3 \\
5.1 \\
4.16 \\
4.98\end{array}$ \\
\hline $\begin{array}{r}13,557 \\
28,629 \\
8,460 \\
11,520 \\
5,500\end{array}$ & $\begin{array}{r}300 \\
1,147 \\
1,000 \\
154 \\
461\end{array}$ & $\begin{array}{r}13,857 \\
29,776 \\
9,460 \\
11,674 \\
5,961\end{array}$ & $\begin{array}{l}6,042 \\
8,000 \\
3,750 \\
1,968 \\
2,509\end{array}$ & $\begin{array}{r}498 \\
2,522 \\
\cdots 24 \\
\ldots \ldots\end{array}$ & $\begin{array}{r}6,540 \\
10,522 \\
3,750 \\
1,992 \\
2,509\end{array}$ & $\begin{array}{l}\ddot{1,527} \\
\ddot{29} \dot{2} \\
229\end{array}$ & $\begin{array}{r}20,397 \\
41,825 \\
13,210 \\
13,958 \\
8,699\end{array}$ & $\begin{array}{l}47.65 \\
34.50 \\
24.92^{5} \\
23.15 \\
35.00\end{array}$ & $\begin{array}{l}917,589 \\
528,799 \\
412,901 \\
158,483\end{array}$ & $\begin{array}{l}5.1 \\
4.56 \\
2.5 \\
3.1 \\
5.48\end{array}$ \\
\hline $\begin{array}{l}71,567 \\
49,681 \\
21,885 \\
57,690 \\
12,818\end{array}$ & $\begin{array}{r}12,717 \\
14,747 \\
3,800 \\
9,350 \\
1,560\end{array}$ & $\begin{array}{l}84,284 \\
64,428 \\
25,685 \\
67,040 \\
14,378\end{array}$ & $\begin{array}{r}23,325 \\
15,796 \\
4,875 \\
15,400 \\
8,119\end{array}$ & $\begin{array}{r}3,087 \\
1,294 \\
1,000 \\
1,800 \\
804\end{array}$ & $\begin{array}{r}26,412 \\
17,090 \\
5,875 \\
17,200 \\
8,923\end{array}$ & $\begin{array}{r}3,391 \\
294 \\
800 \\
2,350 \\
789\end{array}$ & $\begin{array}{r}114,087 \\
81,812 \\
32,360 \\
86,590 \\
24,090\end{array}$ & $\begin{array}{l}15.57 \\
27.35^{5} \\
36.44 \\
18.83 \\
34.12^{5}\end{array}$ & $\begin{array}{r}3,886,815 \\
\cdots 323,110 \\
2,697,724 \\
542,968\end{array}$ & $\begin{array}{l}2.94 \\
3.93 \\
3.2 \\
4.43\end{array}$ \\
\hline $\begin{array}{l}26,074 \\
19,910 \\
33,929 \\
11,432 \\
11,072\end{array}$ & $\begin{array}{l}2,425 \\
2,680 \\
2,211 \\
2,369 \\
4,989\end{array}$ & $\begin{array}{l}28,499 \\
22,590 \\
36,140 \\
13,801 \\
16,061\end{array}$ & $\begin{array}{r}19,895 \\
9,317 \\
14,552 \\
8,375 \\
6,579\end{array}$ & $\begin{array}{r}810 \\
466 \\
1,774 \\
300 \\
2,192\end{array}$ & $\begin{array}{r}20,705 \\
9,783 \\
16,326 \\
8,675 \\
8,771\end{array}$ & $\begin{array}{r}2,059 \\
2,013 \\
1,844 \\
1,552 \\
392\end{array}$ & & $\begin{array}{l}15.44 \\
40.74^{5} \\
23.95 \\
23.00 \\
15.59^{5}\end{array}$ & $\begin{array}{r}1,295,055 \\
680,682 \\
1,103,118 \\
537,000 \\
928,434\end{array}$ & $\begin{array}{l}3.95 \\
5.05 \\
4.92 \\
4.47 \\
2.7\end{array}$ \\
\hline $\begin{array}{r}29,448 \\
8,850 \\
11,400 \\
15,200 \\
7,910\end{array}$ & $\begin{array}{l}7,480 \\
1,728 \\
4,931 \\
4,742 \\
2,069\end{array}$ & $\begin{array}{r}36,928 \\
10,578 \\
16,331 \\
19,942 \\
9,979\end{array}$ & $\begin{array}{l}9,676 \\
3,065 \\
6,286 \\
6,724 \\
4,990\end{array}$ & $\begin{array}{l}324 \\
193 \\
308 \\
642 \\
112\end{array}$ & $\begin{array}{r}10,000 \\
3,258 \\
6,594 \\
7,366 \\
5,102\end{array}$ & $\begin{array}{r}134 \\
599 \\
1,055 \\
1,029\end{array}$ & $\begin{array}{l}46,928^{12} \\
13,960 \\
23,524 \\
28,363 \\
16,110\end{array}$ & $\begin{array}{l}19.39^{5} \\
30.75 \\
16.68 \\
21.81 \\
25.37^{5}\end{array}$ & $\begin{array}{r}1,007,837 \\
529,146 \\
1,190,209 \\
691,284 \\
507,147\end{array}$ & $\begin{array}{l}4.6 \\
2.6 \\
1.9 \\
4.1 \\
3.18\end{array}$ \\
\hline $\begin{array}{l}17,610 \\
36,000 \\
27,398 \\
19,230 \\
34,200\end{array}$ & $\begin{array}{l}2,452 \\
5,400 \\
2,584 \\
2,250 \\
3,495\end{array}$ & $\begin{array}{l}20,062 \\
41,400 \\
29,982 \\
21,480 \\
37,695\end{array}$ & $\begin{array}{r}4,527 \\
10,902 \\
5,697 \\
9,269 \\
8,171\end{array}$ & $\begin{array}{l}845 \\
768 \\
600 \\
315 \\
605\end{array}$ & $\begin{array}{r}5,372 \\
11,670 \\
6,297 \\
9,584 \\
8,776\end{array}$ & $\begin{array}{r}746 \\
2,103 \\
1,418 \\
582 \\
649\end{array}$ & $\begin{array}{l}26,180 \\
55,173^{3} \\
37,697 \\
31,646 \\
47,120\end{array}$ & & $\begin{array}{l}593,254 \\
987,816 \\
575,269 \\
445,019 \\
940,000\end{array}$ & $\begin{array}{l}4.41 \\
5.58 \\
6.55 \\
7.1 \\
5\end{array}$ \\
\hline $\begin{array}{l}48,170 \\
34,269 \\
42,549 \\
30,805 \\
29,225\end{array}$ & $\begin{array}{l}1,600 \\
1,400 \\
3,060 \\
2,016 \\
1,840\end{array}$ & $\begin{array}{l}49,770 \\
35,669 \\
45,609 \\
32,821 \\
31,065\end{array}$ & $\begin{array}{r}12,781 \\
8,931 \\
20,620 \\
13,802 \\
11,789\end{array}$ & $\begin{array}{r}1,793 \\
1,797 \\
791 \\
1,409 \\
798\end{array}$ & $\begin{array}{l}14,574 \\
10,728 \\
21,411 \\
15,211 \\
12,587\end{array}$ & $\begin{array}{l}1,231 \\
1,131 \\
3,582 \\
2,105 \\
2,569\end{array}$ & & & $\begin{array}{r}1,506,633 \\
1,034,237 \\
1,480,572 \\
1,277,243 \\
787,325\end{array}$ & $\begin{array}{l}4.35 \\
5 \\
4.7 \\
3.9 \\
5.87\end{array}$ \\
\hline $\begin{array}{l}33,939 \\
24,890 \\
22,877 \\
10,139 \\
14,770\end{array}$ & $\begin{array}{r}1,521 \\
11,475 \\
1,220 \\
2,033 \\
\ldots \ldots\end{array}$ & $\begin{array}{l}35,460 \\
36,365 \\
24,097 \\
12,172 \\
14,770\end{array}$ & $\begin{array}{r}12,859 \\
11,188 \\
9,517 \\
2,129 \\
3,870\end{array}$ & $\begin{array}{r}2,500 \\
3,227 \\
600 \\
141 \\
212\end{array}$ & $\begin{array}{r}15,359 \\
14,415 \\
10,117 \\
2,270 \\
4,082\end{array}$ & $\begin{array}{r}2,580 \\
2,244 \\
635 \\
7 i 0\end{array}$ & $\begin{array}{l}53,399 \\
34,849 \\
53,024 \\
14,441 \\
18,962\end{array}$ & & $\begin{array}{r}1,040,799 \\
753,747 \\
714,727 \\
424,889 \\
323,052\end{array}$ & $\begin{array}{l}5.1 \\
4.6 \\
7.4 \\
3.4 \\
5.8\end{array}$ \\
\hline $\begin{array}{r}47,110 \\
14,600 \\
5,390 \\
10,497 \\
9,540\end{array}$ & $\begin{array}{r}8,272 \\
2,736 \\
580 \\
342 \\
1,238\end{array}$ & $\begin{array}{r}55,382 \\
17,336 \\
5,970 \\
10,839 \\
10,778\end{array}$ & $\begin{array}{r}21,499 \\
3,779 \\
3,050 \\
2,295 \\
4,947\end{array}$ & $\begin{array}{r}1,600 \\
465 \\
250 \\
173 \\
348\end{array}$ & $\begin{array}{r}23,199 \\
4,914 \\
3,300 \\
2,468 \\
5,295\end{array}$ & $\begin{array}{r}1,768 \\
669 \\
1,924 \\
506 \\
597\end{array}$ & $\begin{array}{l}80,349 \\
22,919 \\
11,194 \\
13,813 \\
16,670\end{array}$ & $\begin{array}{l}27.43^{5} \\
27.64 \\
27.37^{5} \\
25.39 \\
23.00^{5}\end{array}$ & $\begin{array}{r}1,344,549 \\
422,609 \\
285,269 \\
414,700 \\
478,436\end{array}$ & $\begin{array}{l}5.91 \\
5.4 \\
3.92 \\
3.33 \\
3.44\end{array}$ \\
\hline
\end{tabular}


Teachers College Library

\begin{tabular}{|c|c|c|c|c|c|c|c|c|}
\hline ibrary ${ }^{1}$ & $\begin{array}{c}\text { Fiscal } \\
\text { Year } \\
\text { Ending }\end{array}$ & $\begin{array}{l}\text { STUDEN } \\
\text { Total } \\
\text { Under- } \\
\text { grad- } \\
\text { uate }\end{array}$ & $\begin{array}{l}\text { Total } \\
\text { Grad- } \\
\text { uate }\end{array}$ & $\begin{array}{l}\text { LMENT2 } \\
\text { Total } \\
\text { Exten- } \\
\text { sion }\end{array}$ & $\begin{array}{l}\text { Book } \\
\text { Stock }\end{array}$ & $\begin{array}{l}\text { Volumes } \\
\text { Added }\end{array}$ & $\begin{array}{l}\text { News- } \\
\text { papers }\end{array}$ & $\begin{array}{l}\text { Peri- } \\
\text { odicals }\end{array}$ \\
\hline $\begin{array}{l}\text { erford, Southwestern SC ... } \\
\text { Southern Oregon Coll. . . . } \\
\text { e, Eastern Oregon Coll. } \\
\text { a, Oregon Coll. of Educ. } \\
\text { fornia, STC } \ldots \\
\text { f.............. }\end{array}$ & $\begin{array}{r}30 \mathrm{Je} 56 \\
30 \mathrm{Je} 56 \\
30 \mathrm{Je5} 6 \\
30 \mathrm{Je} 56 \\
31 \mathrm{My} 56\end{array}$ & $\begin{array}{r}1,143 \\
769 \\
605 \\
746 \\
1,125\end{array}$ & $\begin{array}{r}95 \\
44 \\
5 \\
\cdots \\
\cdots\end{array}$ & $\begin{array}{l}32 \\
\cdots \cdots \\
\cdots \cdots\end{array}$ & $\begin{array}{l}45,523 \\
33,729 \\
34,996 \\
40,061 \\
30,097\end{array}$ & $\begin{array}{r}1,272 \\
4,111 \\
3,266 \\
3,435 \\
993\end{array}$ & $\begin{array}{r}23 \\
17 \\
12 \\
30 \\
4\end{array}$ & $\begin{array}{l}246 \\
318 \\
405 \\
296 \\
282\end{array}$ \\
\hline 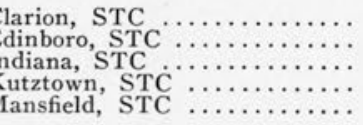 & $\begin{array}{l}31 \text { My } 56 \\
31 \text { My56 } \\
31 \text { My56 } \\
31 \text { My56 } \\
31 \text { My56 }\end{array}$ & $\begin{array}{r}707 \\
635 \\
1,868 \\
1,003 \\
648\end{array}$ & $\begin{array}{l}\cdots \\
\cdots \cdots \\
\cdots \cdots\end{array}$ & $\begin{array}{l}105 \\
\cdots \\
\cdots\end{array}$ & $\begin{array}{l}32,407 \\
35,472 \\
45,121 \\
48,715 \\
35,175\end{array}$ & $\begin{array}{r}2,318 \\
1,483 \\
2,787 \\
2,394 \\
788\end{array}$ & $\begin{array}{r}13 \\
8 \\
11 \\
15 \\
23\end{array}$ & $\begin{array}{l}230 \\
188 \\
270 \\
327 \\
237\end{array}$ \\
\hline 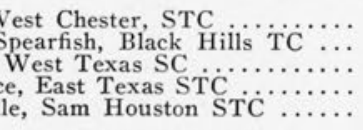 & $\begin{array}{r}31 \mathrm{My} 56 \\
30 \mathrm{Je} 56 \\
31 \mathrm{Ag} 56 \\
31 \mathrm{Ag} 56 \\
31 \mathrm{Ag} 56\end{array}$ & $\begin{array}{r}1,890 \\
588 \\
1,962 \\
1,843 \\
2,141\end{array}$ & $\begin{array}{l}\cdots \\
627 \\
348 \\
181\end{array}$ & $\begin{array}{r}51 \\
500 \\
107 \\
191 \\
322\end{array}$ & $\begin{array}{r}67,614 \\
35,317 \\
52,782 \\
126,920 \\
136,272\end{array}$ & $\begin{array}{l}2,529 \\
890 \\
3,737 \\
3,672 \\
5,570\end{array}$ & $\begin{array}{r}9 \\
18 \\
28 \\
21 \\
10\end{array}$ & $\begin{array}{l}371 \\
260 \\
490 \\
529 \\
590\end{array}$ \\
\hline 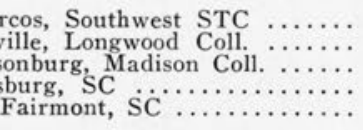 & $\begin{array}{l}31 \mathrm{Ag} 56 \\
30 \mathrm{Je56} \\
\text { 30Je56 } \\
\text { 30Je56 } \\
\text { 30Je56 }\end{array}$ & $\begin{array}{r}1,943 \\
755 \\
1,141 \\
1,547 \\
1,193\end{array}$ & $\begin{array}{r}214 \\
19 \\
24 \\
62 \\
\cdots\end{array}$ & $\begin{array}{l}129 \\
\cdots \\
\because 559^{16} \\
\cdots\end{array}$ & $\begin{array}{l}86,728 \\
64,800 \\
78,443 \\
63,245 \\
42,848\end{array}$ & $\begin{array}{l}2,523 \\
2,178 \\
4,752 \\
2,755 \\
1,333\end{array}$ & $\begin{array}{r}14 \\
12 \\
13 \\
20 \\
6\end{array}$ & $\begin{array}{l}488 \\
320 \\
396 \\
701 \\
292\end{array}$ \\
\hline 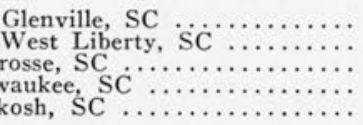 & $\begin{array}{r}30 \mathrm{Je56} \\
30 \mathrm{Je56} \\
30 \mathrm{Je56} \\
31 \mathrm{Ag} 56 \\
30 \mathrm{Je56}\end{array}$ & $\begin{array}{r}602 \\
1,103 \\
1,334 \\
2,305 \\
1,164\end{array}$ & $\begin{array}{l}\cdots \cdots \\
\cdots \\
75 \\
\cdots\end{array}$ & $\begin{array}{r}\cdots 2 \dot{2} \\
126 \\
30 \dot{30}\end{array}$ & $\begin{array}{l}35,492 \\
31,203 \\
52,497 \\
82,549 \\
55,243\end{array}$ & $\begin{array}{l}1,268 \\
1,689 \\
2,821 \\
4,004 \\
2,044\end{array}$ & $\begin{array}{l}26 \\
14 \\
12 \\
20 \\
14\end{array}$ & $\begin{array}{l}273 \\
341 \\
293 \\
502 \\
278\end{array}$ \\
\hline 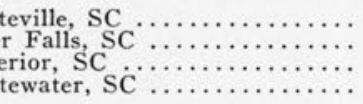 & $\begin{array}{l}\text { 30 Je56 } \\
30 \mathrm{Je} 56 \\
\text { 30Je56 } \\
\text { 30Je56 }\end{array}$ & $\begin{array}{r}912 \\
914 \\
1,038 \\
1,067\end{array}$ & $\begin{array}{l}\cdots \\
\cdots \cdots \\
\cdots\end{array}$ & $\begin{array}{l}261 \\
126 \\
163 \\
589\end{array}$ & $\begin{array}{l}38,481 \\
41,293 \\
60,084 \\
56,018\end{array}$ & $\begin{array}{l}2,208 \\
1,769 \\
2,293 \\
1,800\end{array}$ & $\begin{array}{r}29 \\
18 \\
10 \\
8\end{array}$ & $\begin{array}{l}310 \\
320 \\
428 \\
402\end{array}$ \\
\hline & & $\begin{array}{r}6,776 \\
1,133 \\
172 \\
94\end{array}$ & $\begin{array}{r}1,553 \\
119 \\
5 \\
57\end{array}$ & $\begin{array}{c}1,577 \\
311.5 \\
8 \\
66\end{array}$ & $\begin{array}{r}174,577 \\
53,073 \\
13,735 \\
93\end{array}$ & $\begin{array}{r}13,041 \\
2,524 \\
375 \\
94\end{array}$ & $\begin{array}{r}46 \\
13 \\
2 \\
93\end{array}$ & $\begin{array}{c}1,199 \\
346.5 \\
128 \\
94\end{array}$ \\
\hline
\end{tabular}

66. Oklahoma, Weatherford, Southwestern $\mathrm{SC}$ 67. Oregon, Ashland, Southern Oregon Coll. 68. Oregon, La Grande, Eastern Oregon Coll. . 69. Oregon, Monmouth, Oregon Coll. of Educ. . 70. Pennsylvania, California, STC ...........

72. Pennsylvania, Edinboro, STC $\ldots \ldots \ldots$

73. Pennsylvania, Indiana,

74. Pennsylvani

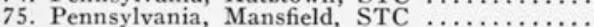

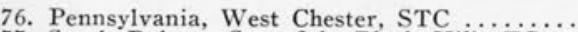

77. South Dakota, Spearfish, Black Hills TC ...

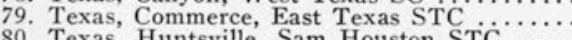

81. Texas, San Marcos, Southwest STC

82. Virginia, Farmvill

83. Virginia, Harrisonburg,

84. Vir

84. Virginia, Peters

85. West Virginia, Fairmont, $\mathrm{SC} \ldots \ldots \ldots$

86. West Virginia, Glenville, SC

87. West Virginia, West Liberty, SC

88. Wisconsin, LaCrosse, SC

89. Wisconsin, Milwauk,

90. Wisconsin, Oshkosh,

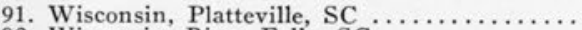

92. Wisconsin, River Falls, SC W. W .

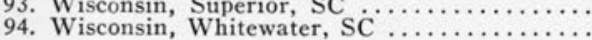

High

Median

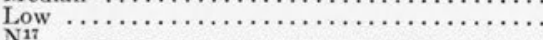

1. Central library and all agencies. 2. Includes all students taking courses of college grade on campus. 3 . Includes supplies, transportation and all other noncapital expenditures not specifically reported. 4 . Exclusive of capital expenditures and those for auxiliary enterprises such as dormitories, cafeterias, etc. 5. Extension enrollment not included in per student expenditure. 6. Not reported or not available. 7 . 2,082 additional volumes pur.

\section{Teachers College Library}

\begin{tabular}{|c|c|c|c|c|c|c|c|c|c|}
\hline \multirow[b]{2}{*}{ Library $^{2}$} & \multirow[b]{2}{*}{$\underset{\text { brarian }}{\mathrm{Li}-}$} & \multicolumn{2}{|c|}{$\begin{array}{c}\text { Associate or } \\
\text { AssistANT } \\
\text { CHIEF LIBRARIAN }\end{array}$} & \multirow{2}{*}{$\begin{array}{l}\text { DEPART } \\
\text { Mini- } \\
\text { mum }\end{array}$} & \multirow{2}{*}{$\begin{array}{l}\text { TMENT } \\
\text { Maxi- } \\
\text { mum }\end{array}$} & \multirow{2}{*}{$\begin{array}{l}\text { HEADS } \\
\text { Num- } \\
\text { ber }\end{array}$} & \multicolumn{3}{|c|}{$\begin{array}{l}\text { HEAD LIBRARIAN, } \\
\text { SCHOOL, CoLLEGE, } \\
\text { AND DEPARTMENTI } \\
\text { LIBRARIES }\end{array}$} \\
\hline & & $\begin{array}{l}\text { Mini- } \\
\text { mum }\end{array}$ & $\begin{array}{l}\text { Maxi- } \\
\text { mum }\end{array}$ & & & & $\begin{array}{l}\text { Mini- } \\
\text { mum }\end{array}$ & & \\
\hline & 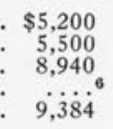 & $\begin{array}{r}\$ 2,400 \\
5,000 \\
\ldots \ldots \\
5,496\end{array}$ & $\begin{array}{r}\$ 4,800 \\
\ldots \ldots \\
\ldots \ldots \\
6,672\end{array}$ & $\begin{array}{r}\$ \ldots \\
5,396 \\
4,508\end{array}$ & $\begin{array}{l}6,060 \\
6,060\end{array}$ & $\begin{array}{l}. . \\
\mathrm{i} \\
4 \\
5\end{array}$ & $\begin{array}{l}\$_{5,232} \\
\ddot{4,308}\end{array}$ & $\begin{array}{r}\$ \ldots \\
\ldots \ldots \\
5,496\end{array}$ & ; \\
\hline & $\begin{array}{l}8,9 \\
8,9 \\
9,3 \\
4,6 \\
5,5\end{array}$ & & $\begin{array}{l}6,6 \\
6,6\end{array}$ & $\begin{array}{l}4,980 \\
4,980\end{array}$ & $\begin{array}{l}6,060 \\
6,060\end{array}$ & $\begin{array}{r}2 \\
4 \\
\therefore \\
.\end{array}$ & $\begin{array}{r}4,512 \\
4,512 \\
\ldots \ldots\end{array}$ & $\begin{array}{l}5,496 \\
5,496 \\
\ldots \ldots\end{array}$ & \\
\hline ...... & $\begin{array}{c}5,900 \\
9,680^{5} \\
\cdots{ }^{5} \\
7,200^{5}\end{array}$ & $\ldots$ & & \begin{tabular}{l}
4,400 \\
5,940 \\
\hdashline, $028^{8}$ \\
4,440
\end{tabular} & $\begin{array}{l}4,500 \\
6,820 \\
8 \quad \dddot{6,743^{8}} \\
5,520\end{array}$ & $\begin{array}{r}2 \\
5 \\
.8 \\
3\end{array}$ & \begin{tabular}{l}
4,700 \\
4,860 \\
\hdashline,$\dddot{22} \dot{8}$
\end{tabular} & ${ }_{5,060^{5}}$ & \\
\hline …. & $\begin{array}{l}8,000{ }^{5} \\
6,7000^{5} \\
5,0000^{5}\end{array}$ & $\ldots$ & $\begin{array}{r}\ldots . \\
\ldots \ldots \\
4,500\end{array}$ & $\begin{array}{c}5,100 \\
4,707^{5} \\
\ldots \ldots\end{array}$ & $\begin{array}{c}5,900 \\
\quad 3,{ }^{5} \\
5 \ldots 8^{5} \\
\ldots \ldots\end{array}$ & $\begin{array}{l}5 \\
8 \\
5 \\
3 \\
.\end{array}$ & $\begin{array}{c}\ldots \ldots \\
4,250^{5} \\
\ldots \cdots^{5}\end{array}$ & $\ldots$ & 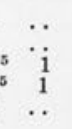 \\
\hline & $\begin{array}{l}4,500 \\
5,100 \\
8,050 \\
9,125 \\
5,417\end{array}$ & $\begin{array}{c}3,000 \\
7,375 \\
7,125 \\
\ldots \ldots\end{array}$ & $\begin{array}{c}3,700 \\
\cdots{ }^{5} \\
7,500^{5} \\
\ldots \ldots\end{array}$ & $\begin{array}{l}3,700 \\
6,000 \\
\ldots \ldots\end{array}$ & $\begin{array}{r}4,400 \\
6,375 \\
\quad 4,282\end{array}$ & $\begin{array}{r}3 \\
5 \\
i\end{array}$ & $\ldots^{5}$ & 4,000 & \\
\hline
\end{tabular}

1. Alabama, Troy, STC $\ldots \ldots \ldots \ldots \ldots \ldots \ldots \ldots+\ldots, 200$

. Arizona, Flagstaff, STC ............. 5,500

3. California, Arcata, Humboldt SC ........ 8,940

4. California, Chico,

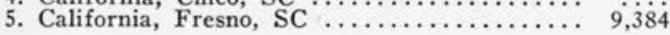

6. California, Long Beach, SC ............ 8,940

7. California, Sacramento, SC

8. California, San Francisco, SC ......... 9,384

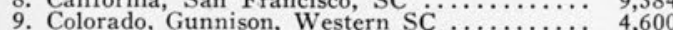

10. Connecticut, Willimantic, STC ..........

11. Georgia, Collegeboro, Ga. TC ......... 5,900

12. Illinois, Charleston, Eastern SC $\ldots \ldots \ldots \ldots \ldots .9,680$

13. Illinois, DeKalb, Northern SC $\ldots \ldots \ldots \ldots$

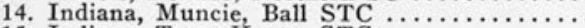

15. Indiana, Terre Haute, STC

16. Iowa, Cedar Falls, STC

18. Kansas, Emporia, STC

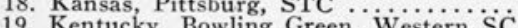

20. Kentucky, Morehead SC .......... 500

21. Kentucky, Murray, SC .............. 4,500

22. Kentucky, Richmond, Eastern SC ........ 5,100

23. Louisiana, Lafayette, Southwestern La. Inst. 8,050

25. Maryland, Frostburg, STC ........... 5,417

Head Librarian, COLLEGE,

ART MENT HEAdS

$\ldots \ldots \ldots \ldots$

$5,496 \quad \cdots \cdots$

$4,508 \quad 6,060 \quad 4$

$\ddot{4} 9 \dot{80} \quad \dot{0}, 060$

$4,980 \quad 6,060$

$4,400 \quad 4,500$

5,520

$100 \quad 5,900 \quad 5$

$707 \quad 5,368$

.... 4,282 i

$3,572 \quad \ldots 0^{5}$ 
Statistics, $1955^{-5} 6$ (Cont.)

\begin{tabular}{|c|c|c|c|c|c|c|c|c|c|c|c|}
\hline $\begin{array}{c}\text { Staff } \\
\text { Salaries }\end{array}$ & $\begin{array}{l}\text { Student } \\
\text { Service }\end{array}$ & $\begin{array}{l}\text { LIBRAR } \\
\text { Total } \\
\text { Staff } \\
\text { Salaries } \\
\text { and } \\
\text { Student } \\
\text { Service }\end{array}$ & $\begin{array}{c}\text { Books } \\
\text { and } \\
\text { Related } \\
\text { Materials }\end{array}$ & Binding & $\begin{array}{c}\text { Total } \\
\text { for } \\
\text { Books } \\
\text { and } \\
\text { Binding }\end{array}$ & $\begin{array}{l}\text { Other } \\
\text { Operating } \\
\text { Expendi- }_{\text {tures }^{3}}\end{array}$ & $\begin{array}{c}\text { Total } \\
\text { Operat- } \\
\text { ing Ex- } \\
\text { pendi- } \\
\text { tures }\end{array}$ & $\begin{array}{l}\text { Per Stu- } \\
\text { dent Op- } \\
\text { erating } \\
\text { Expendi- } \\
\text { tures }\end{array}$ & $\begin{array}{l}\text { Total Edu- } \\
\text { cational } \\
\text { and Gen- } \\
\text { eral Insti- } \\
\text { tutional } \\
\text { Expendi- } \\
\text { tures }\end{array}$ & $\begin{array}{c}\text { Ratio of } \\
\text { Library } \\
\text { Expendi } \\
\text { tures to } \\
\text { Total } \\
\text { (Per Cen }\end{array}$ & \\
\hline $\begin{array}{r}10,010 \\
23,369 \\
15,660 \\
10,047 \\
9,114\end{array}$ & $\begin{array}{l}2,318 \\
3,530 \\
9,018 \\
8,060 \\
2,623\end{array}$ & $\begin{array}{l}12,328 \\
26,899 \\
24,678 \\
18,107 \\
11,737\end{array}$ & $\begin{array}{r}5,441 \\
8,940 \\
11,029 \\
11,440 \\
1,019\end{array}$ & $\begin{array}{r}419 \\
1,023 \\
1,154 \\
1,018 \\
1,480\end{array}$ & $\begin{array}{r}5,860 \\
9,963 \\
12,183 \\
12,458 \\
2,499\end{array}$ & $\begin{array}{r}7,640 \\
3,540 \\
1,764 \\
1,707 \\
385\end{array}$ & $\begin{array}{l}25,828 \\
40,402 \\
38,625 \\
32,272 \\
14,621\end{array}$ & $\begin{array}{l}20.34 \\
42.69 \\
63.32 \\
43.26 \\
13.00\end{array}$ & $\begin{array}{l}629,610 \\
536,653 \\
460,300 \\
601,565 \\
821,004\end{array}$ & $\begin{array}{l}4.1 \\
7.5 \\
8.39 \\
5.36 \\
1.78\end{array}$ & $\begin{array}{l}66 \\
67 \\
68 \\
69 \\
70\end{array}$ \\
\hline $\begin{array}{l}12,820 \\
12,160 \\
24,837 \\
29,720 \\
18,052\end{array}$ & $\begin{array}{l}4,780 \\
4,206 \\
3,387 \\
3,000 \\
2,133\end{array}$ & $\begin{array}{l}17,600 \\
16,366 \\
28,224 \\
32,720^{15} \\
20,185\end{array}$ & $\begin{array}{l}3,137 \\
7,690 \\
8,664 \\
6,431 \\
4,052\end{array}$ & $\begin{array}{r}225 \\
583 \\
81 \\
82\end{array}$ & $\begin{array}{l}3,137 \\
7,915 \\
9,247 \\
6,512 \\
4,134\end{array}$ & $\begin{array}{r}1,550 \\
285 \\
1,839 \\
\cdots 407\end{array}$ & $\begin{array}{l}22,287 \\
24,566 \\
39,310 \\
39,232 \\
24,726\end{array}$ & $\begin{array}{l}31.52 \\
38.68^{5} \\
21.04 \\
39.11 \\
38.15\end{array}$ & $\begin{array}{r}378,182 \\
621,074 \\
1,158,000 \\
715,662 \\
701,679\end{array}$ & $\begin{array}{l}5.9 \\
3.96 \\
3.4 \\
5.5 \\
3.5\end{array}$ & $\begin{array}{l}73 \\
74 \\
75\end{array}$ \\
\hline $\begin{array}{r}29,284 \\
5,700 \\
29,600 \\
34,642 \\
24,452\end{array}$ & $\begin{array}{r}2,762 \\
1,997 \\
5,786 \\
12,078 \\
3,589\end{array}$ & $\begin{array}{r}32,046 \\
7,697 \\
35,386 \\
46,720 \\
28,041\end{array}$ & $\begin{array}{r}5,528 \\
3,830 \\
11,129 \\
14,354 \\
12,771\end{array}$ & $\begin{array}{r}605 \\
\dddot{977} \\
1,639 \\
1,053\end{array}$ & $\begin{array}{r}6,133 \\
3,830 \\
12,106 \\
15,993 \\
13,824\end{array}$ & $\begin{array}{r}324 \\
350 \\
2,470 \\
883 \\
1,199\end{array}$ & $\begin{array}{l}38,503 \\
11,876 \\
49,962 \\
63,596 \\
43,064\end{array}$ & $\begin{array}{l}19.83 \\
20.20^{5} \\
18.53 \\
28.98^{5} \\
18.54^{5}\end{array}$ & $\begin{array}{r}1,219,000 \\
393,083 \\
1,020,305 \\
1,368,641 \\
1,327,507\end{array}$ & $\begin{array}{l}3.16 \\
3.02 \\
4.9 \\
4.65 \\
3.24\end{array}$ & $\begin{array}{l}77 \\
78 \\
79\end{array}$ \\
\hline $\begin{array}{l}27,465 \\
20,755 \\
26,342 \\
40,408 \\
17,181\end{array}$ & $\begin{array}{l}6,039 \\
2,462 \\
3,714 \\
5,034 \\
1,905\end{array}$ & $\begin{array}{l}33,504 \\
23,217 \\
30,056 \\
45,442 \\
19,086\end{array}$ & $\begin{array}{r}10,533 \\
6,248 \\
12,362 \\
12,704 \\
3,831\end{array}$ & $\begin{array}{r}1,252 \\
1,854 \\
1,226 \\
529\end{array}$ & $\begin{array}{r}10,533 \\
7,500 \\
14,216 \\
13,930 \\
4,360\end{array}$ & $\begin{array}{r}2,170 \\
715 \\
1,854 \\
2,229 \\
5,993\end{array}$ & $\begin{array}{l}46,207 \\
31,432 \\
46,126 \\
61,601 \\
29,439\end{array}$ & $\begin{array}{l}20.21 \\
40.61 \\
39.60 \\
28.41 \\
24.67\end{array}$ & $\begin{array}{r}1,188,862 \\
691,511 \\
778,129 \\
1,734,120 \\
501,301\end{array}$ & $\begin{array}{l}3.9 \\
4.54 \\
5.92 \\
3.55 \\
5.87\end{array}$ & $\begin{array}{l}31 \\
33 \\
34\end{array}$ \\
\hline $\begin{array}{r}10,081 \\
9,360 \\
25,675 \\
36,634 \\
25,500\end{array}$ & $\begin{array}{r}1,477 \\
2,188 \\
400 \\
4,600 \\
3,000\end{array}$ & $\begin{array}{l}11,558 \\
11,548 \\
26,075 \\
41,234 \\
28,500\end{array}$ & $\begin{array}{r}4,005 \\
4,611 \\
8,456 \\
17,646 \\
8,800\end{array}$ & $\begin{array}{r}309 \\
616 \\
613 \\
2,090 \\
750\end{array}$ & $\begin{array}{r}4,314 \\
5,227 \\
9,069 \\
19,736 \\
9,550\end{array}$ & $\begin{array}{r}842 \\
408 \\
1,156 \\
800 \\
2,000\end{array}$ & $\begin{array}{l}16,714 \\
17,183 \\
36,300 \\
61,770 \\
40,050\end{array}$ & $\begin{array}{l}27.76 \\
15.58^{5} \\
27.20^{5} \\
25.95 \\
33.84^{5}\end{array}$ & $\begin{array}{r}316,591 \\
343,790 \\
865,329 \\
1,276,107 \\
758,598\end{array}$ & $\begin{array}{l}5.28 \\
4.99 \\
4.19 \\
4.8 \\
5.27\end{array}$ & 78 \\
\hline $\begin{array}{l}18,435 \\
22,347 \\
20,500 \\
20,944\end{array}$ & $\begin{array}{l}3,024 \\
1,170 \\
5,007 \\
7,164\end{array}$ & $\begin{array}{l}21,459 \\
23,517 \\
25,507 \\
28,108\end{array}$ & $\begin{array}{l}8,267 \\
8,178 \\
5,922 \\
8,351\end{array}$ & $\begin{array}{l}561 \\
600 \\
925 \\
853\end{array}$ & $\begin{array}{l}8,828 \\
8,778 \\
6,847 \\
9,204\end{array}$ & $\begin{array}{r}618 \\
200 \\
1,700 \\
\ldots \ldots\end{array}$ & $\begin{array}{l}30,905 \\
32,495 \\
34,054 \\
37,312\end{array}$ & $\begin{array}{l}33.89^{5} \\
35.55^{5} \\
32.80^{5} \\
34.96^{5}\end{array}$ & $\begin{array}{l}538,972 \\
562,496 \\
633,824 \\
552,710\end{array}$ & $\begin{array}{l}5.73 \\
5.77 \\
5.37 \\
6.75\end{array}$ & $\begin{array}{l}91 \\
92 \\
93 \\
94\end{array}$ \\
\hline $\begin{array}{r}134,096 \\
23,123 \\
5,390 \\
94\end{array}$ & $\begin{array}{r}34,907 \\
3,495 \\
154 \\
93\end{array}$ & $\begin{array}{r}169,003 \\
26,487 \\
5,961 \\
94\end{array}$ & $\begin{array}{r}43,531 \\
91,045 \\
1,019 \\
94\end{array}$ & $\begin{array}{r}9,234 \\
1,077 \\
24 \\
89\end{array}$ & $\begin{array}{c}49,256 \\
9981.5 \\
1,992 \\
94\end{array}$ & $\begin{array}{cc} & 10,872 \\
5 & 1472.5 \\
110 \\
\\
& 88\end{array}$ & $\begin{array}{c}216,971 \\
37,504.5 \\
8,699 \\
94\end{array}$ & $\begin{array}{r}63.83 \\
29.07 \\
12.96 \\
94\end{array}$ & $\begin{array}{r}4,019,807 \\
782,727 \\
158,483 \\
90\end{array}$ & $\begin{array}{l}8.39 \\
4.6 \\
1.78 \\
91\end{array}$ & $\begin{array}{r}\text { High } \\
\text { Iedian } \\
\text { Low } \\
\mathrm{N}^{17}\end{array}$ \\
\hline
\end{tabular}

chased by departmental funds. 8. Includes A-V expenditures. 9. Includes on- and off-campus students. 10. Excludes Educational Service Library and Campus School. 11. Not included in per student expenditure. 12. Prorated over biennium. 13. Includes part-time students, 14. Estimated figures, 15. Excludes summer session expenses. 16. Includes 158 two-year vocational students. 17. Number of libraries on which high, median and low are based.

\section{Salary Statistics, I 955-1956}

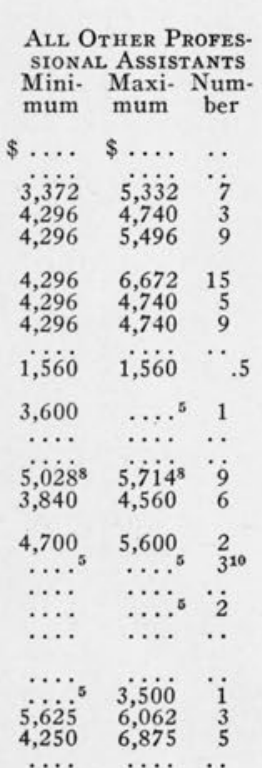

\begin{tabular}{|c|c|c|}
\hline $\begin{array}{l}\text { ALL } \\
\text { SIONA }\end{array}$ & $\begin{array}{l}\text { NoNPRO } \\
\text { ASSIST }\end{array}$ & $\begin{array}{l}\text { FES- } \\
\text { ANTS }\end{array}$ \\
\hline $\begin{array}{l}\text { Mini- } \\
\text { mum }\end{array}$ & $\begin{array}{l}\text { Maxi- } \\
\text { mum }\end{array}$ & $\underset{\text { ber }}{\text { Num }}$ \\
\hline$\$ \cdots_{5}$ & $\$ \ldots$ & \\
\hline $\begin{array}{l}3,216 \\
2,771\end{array}$ & $\begin{array}{l}3,9000 \\
4,740\end{array}$ & \\
\hline 3,060 & 4,740 & 13.5 \\
\hline 3,216 & 4,092 & ${ }_{8}^{17.5}$ \\
\hline 3,060 & 4,740 & 14 \\
\hline $\begin{array}{l}1,920 \\
2,940\end{array}$ & 3,300 & $\begin{array}{l}1 \\
2\end{array}$ \\
\hline $1, \ddot{9} \dot{20}$ & 2,640 & 3.5 \\
\hline $\begin{array}{l}2,220 \\
2,190\end{array}$ & $\begin{array}{l}3,285 \\
3,060\end{array}$ & $\begin{array}{r}12 \\
4\end{array}$ \\
\hline 2,240 & 3,000 & 6 \\
\hline 1,980 & 2,2992 & 2 \\
\hline$\dot{1,600}$ & $\cdots$ & 1 \\
\hline 1,2000 & $\ddot{2,000}$ & \\
\hline $\begin{array}{l}1,680 \\
2,280\end{array}$ & 3,000 & \\
\hline$\ldots$ & 4,282 & 2 \\
\hline
\end{tabular}

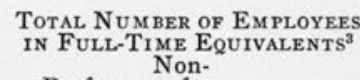

Working

Profes-
sionals siofes-
sionals

Fiscal

Hours of

Assistance

\begin{tabular}{rrr}
3.5 & \multicolumn{1}{c}{} & 3.5 \\
3 & $\mathrm{i}$ & 4 \\
8 & 3.5 & 11.5 \\
9 & 3.5 & 12.5 \\
15 & 11.5 & 26.5
\end{tabular}

2,316

1,808

10,368

1,856

1,999

4,650
10,208

10,208
16,094

$\begin{array}{rcl}15 & 17.5 & 32.5 \\ 12 & 8 & 20 \\ 22 & 14 & 36 \\ 2 & 1 & 3\end{array}$

$\begin{array}{lll}2 & 1 & 3 \\ 1.5 & 2 & 3.5\end{array}$

1,200

$1,784^{\circ}$

1,505

20,400

21,727

654
1,512

$\begin{array}{lll}5 & \ddots & 5 \\ 8 & 3.5 & 11.5\end{array}$

$\begin{array}{lll}i 9 & \mathrm{i} 2 & 3 \mathrm{i} \\ 11 & 2.5 & 13.5\end{array}$

$9,200^{7}$

5,966

1,628

14,203

$1, \dddot{6} 80$

$49,86 \overline{7}$

6,500

$\begin{array}{lllll}8.33 & 6 & 14.33^{\circ} & 1,665\end{array}$

17,685

1,624

$\begin{array}{cccc}12 & 2.5 & 14.5 & 1,724 \\ 7 & 2 & 9 & 1,920 \\ 6.5 & 2.5 & 9 & 1,760\end{array}$

27,254

12,141
4,374

$\begin{array}{rlcl}4 & & 4 & 1,508 \\ 6 & \mathrm{i} & 7 & 1,706 \\ 10 & 4 & 14 & 1,600 \\ 7 & 4.5 & 11.5 & 1,599 \\ 3 & 1.83 & 4.83 & 1,597\end{array}$

8,000

8,800

34,707
18,893

$18,89{ }^{\circ}$ 
Associate or

Assistant

Chief Librarian
Mini- Maxi-

Library ${ }^{2}$

Li-

brarian

26. Maryland, Salisbury, STC

5,400

28. Massachusetts, Fitchburg, STC ........ 5, 5,340

29. Massachusetts, Framingham, STC ............ 6,300

30. Massachusetts, North Adams, STC .......... 5,500

31. Michigan, Kalamazoo, Western Mich. Coll. .

32. Michigan, Mt. Pleasant, Central Mich. Coll.

33. Michigan, Marquette, Northern Mich. Coll.

34. Michigan, Ypsilanti, Eastern Mich. Coll. ...

36. Minnesota, Mankato, STC

37. Minnesota, Mankato, STC $\ldots \ldots \ldots \ldots \ldots$.

38. Minnesota, St. Cloud, STC $\ldots \ldots \ldots \ldots \ldots \ldots \ldots \ldots$

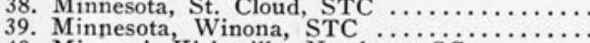

40. Missouri, Kirksville, Northeast $\mathrm{SC} \ldots \ldots \ldots$

41. Missouri, Springfield, Southwest SC ......

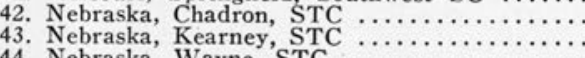

44. Nebraska, Wayne, STC

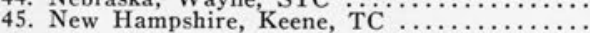

46. New Jersey, Glassboro, STC

47. New Jersey, Montclair, STC $\ldots \ldots \ldots \ldots \ldots$

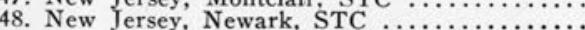

49. New Jersey, Paterson, STC

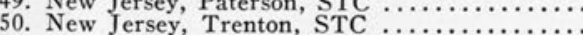

51. New York, Albany, STC

52. New York, Brockport, STC $\ldots \ldots \ldots \ldots \ldots \ldots$

53. New York, Buffalo, STC

54. New York, Cortland, STC $\ldots \ldots \ldots \ldots \ldots \ldots \ldots$

55. New York, Oneonta, STC

56. New York, Oswego, STC

57. New York, Plattsburgh, STC

58. North Carolina, Boone, Appalachian ST $_{\mathrm{C}} \ldots$

59. North Carolina, Elizabeth City, STC .......

0. North Carolina, Fayetteville, STC ..........

61. North Carolina, Greenville, E. Carolina Coll

63. North Darolina, Winston-Salem

64. North Dakota, Valley City, STC $\ldots \ldots \ldots \ldots$

65. Oklahoma, Alva, Northwestern $S C \ldots \ldots \ldots$

66. Oklahoma, Weatherford, Southwestern SC ...

68. Oregon, Ashland, Southern Ore. Coll. ..........

69. Oregon, Monmouth, Ore. Coll. of Educ. ....

71. Pennsylvania, Clarion, STC

72. Pennsylvania, Edinboro, STC $\cdots \ldots \ldots \ldots \ldots$

73. Pennsylvania, Indiana, STC

74. Pennsylvania, Kutztown, STC $\ldots \ldots \ldots \ldots$

75. Pennsylvania, Mansfield, STC

76. Pennsylvania, West Chester, STC

8,050

7,700
7,675
5,120

5,120

$6,020^{12}$

$3,700^{13}$

$5,20 \dot{8}^{14}$

5,900

5,243

5,268

5,500

, 300

6,600

6,240

6,660

7,140
$7,950^{18}$

5,976

7,206

6,906

7,206

7,206

6,906

6,210

5,696
4,063

4,596

7,669

4,794

4,400

4,600

$5,355^{16}$

5,500

6,345

5,397

5,370

6,400

5,700

6,050

5,900
6,710

South Dakota, Spearfish, Black Hills TC .... 6,120

8. Texas, Canyon, West Texas SC $\ldots \ldots \ldots$

9. Texas, Commerce, E. Texas STC

6,420

7, $\dot{1} \dot{5} \dot{3}$

81. Texas, San Marcos, Southwest STC

82. Virginia, Farmville, Longwood Coll

83. Virginia, Harrisonburg, Madison Coll. .....

84. Virginia, Petersburg, Va. SC

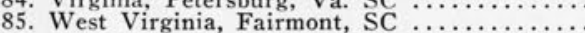

86. West Virginia, Glenville, SC

87. West Virginia, West Liberty, $\ddot{\text { S }} \ldots \ldots \ldots \ldots$

88. Wisconsin, LaCrosse, SC

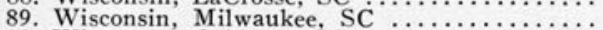

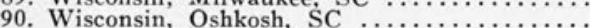

92. Wisconsin, River Falls, SC $_{\ldots \ldots \ldots \ldots \ldots \ldots} \ldots \ldots \ldots \ldots$

93. Wisconsin, Superior, S

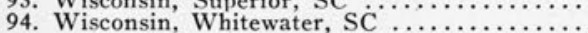

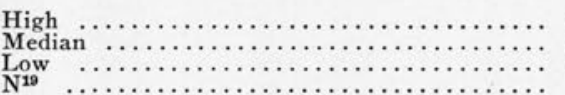

mum mum

$\ddot{3, j} \dot{\bar{z}} \dot{4} \ddot{2} \ddot{2} \dot{2}$

5,220

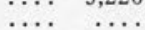

$6,850^{11} \ldots{ }^{5}$

$\cdots{ }^{5} \quad \cdots{ }^{\circ}$

$5,575 \quad \ldots$.

4,896

4.نं4

6,080

4,740

$5,160 \quad 5,243$

5,200

5,150

3,760

$5,460 \quad 6,660$

$\ldots \ldots+\cdots$

$\ldots \ldots$

$4,698 \quad 5,748$

$4,698 \quad 5,526$

$4,698 \quad 5,748$

$4,698 \quad 5,748$

$4,698 \quad 5,748$

$4,698 \quad 5,748$

$5,395 \quad 5,748$

$\cdots{ }^{\prime} \quad \ldots$

4,176

3,800

5,310

4,510

...

$4,6 \dot{5} 0 \quad 4,650$

5,800

5,700

$4,000 \quad 5,875$

$4,300 \quad 5,800$

2,450

. 800

$4,500 \quad 6,500$

$5, \dot{2} \dot{20}$

…

... 4,60

4,9்0

\section{$4,150 \quad \ldots$}

3,800

$5,725 \quad \cdots$

$\ldots+\quad \ldots$

$7,375 \quad 7,500$

$\begin{array}{ll}7,375 & 7,500 \\ 4,698 & 5,748 \\ 2,400 & 3,700\end{array}$

Depart ment Heads

Mini- Maxi- Num-

Head Librarian,

School, College,

ND DEPARTMENTAL

LIBRARIES

Mini- Maxi-Num

mum mum ber

mum ber

$\begin{array}{lll}\cdots & \cdots & \cdots \\ \cdots & \cdots\end{array}$

$\begin{array}{lll}\ldots, 400 \quad 1 & 1\end{array}$

... $\ldots$

$6,200 \quad 6,400$

$5,200 \quad 6,600$

$5,000 \quad 6,200 \quad i 35,000 \quad \ldots \ldots 5 ;$

$5,500 \quad 6,000$

$3,600 \quad 6,180 \quad 4^{15} \quad 4,020 \quad 4,9200 \quad$ i

$\begin{array}{llllll}\cdots & \cdots & \cdots & \cdots & \ldots & \cdots \\ \cdots & \cdots & \cdots & \ldots & \ldots & \cdots\end{array}$

$\cdots \cdots$

$\ddot{3,840} \quad \dot{4}, 790 \quad$ i

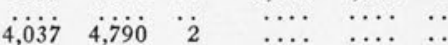

$4,0000 \quad 4,03 \dot{3}$

$\cdots \cdots$

$4,500 \quad 5,425$

$4,872 \quad \ldots \ldots \quad 1$

$\cdots \cdots$

$\cdots+\cdots$

\section{.}

$\cdots+\cdots$

$\ddot{1,3000} \quad \ddot{1, j 000} \quad \cdots$

\section{4, $32 \dot{2}$}

$3,500 \quad 4,600$

\begin{tabular}{l}
$4,470 \quad 4,900$ \\
\hline
\end{tabular}

$3,240 \quad 4,400$

$4,320 \quad 4,320$

$3,744 \quad 4,320$

4,300

... ${ }^{8}$

...

$\cdots{ }^{\circ}$

$\begin{aligned} & 5,215 \\ & 4,615\end{aligned} \quad \therefore, 54 \dot{0}^{5}$

$\begin{array}{cc}4,615 & 5,540 \quad 2 \\ \ldots & \ldots\end{array}$

$4,600 \quad 5,200 \quad 3$

$\ldots$

…

$6,200 \quad 6,820$

4,560
5,520

$3,240 \quad 4,039$

2938

$\begin{array}{ll}6,228 & 5,496 \\ 4,600 & 4,990\end{array}$

$1,500 \quad 1,500$

1. Salary data reported as of September 1, 1956. 2. Central library and all agencies. 3. Excludes student assistants and building maintenance staff. 4. Excludes vacation and legal holidays. 5. Not reported or not available. 6. Salary range of $\$ 7,728-9,384$ reported. 7 . Total hours for all staff. 8 . Includes one-seventh of academic year salary for summer session. 9. Includes two part-time professionals and one part-time clerical assistant. 10. 
Salary Statistics, $1955^{-5} 6^{6^{1}}$ (Cont.)

All Other Profes-

SIONAL Assistants
Mini- Maxi- Num-

mum mum ber

$\begin{array}{lrr}\ldots{ }^{5} & 7,475 & 2 \\ \cdots & \cdots & \ldots\end{array}$

$\begin{array}{lll}\cdots & \cdots & \cdots \\ \cdots & \cdots & \cdots\end{array}$

$\begin{array}{lll}\cdots & \cdots & \cdots\end{array}$

$3,600 \quad 6,200 \quad 4$

$4,400 \quad 5,400 \quad{ }^{5}{ }^{5}$

$\begin{array}{ccc}4,400 & 4,460 & 4 \\ \cdots & \cdots & \end{array}$

$\begin{array}{lll}4,284 & \ldots & 5 \\ 3,672 & \ldots & 4 \\ 5,120 & \ldots & 1 \\ & & 1\end{array}$

$\ddot{3,600} \quad \dot{4,000} \quad \ddot{3}$

$\begin{array}{lll}\ldots & \ldots & \ldots \\ \cdots & \ldots & \ldots\end{array}$

$\begin{array}{lll}\cdots & \cdots & \cdots \\ \cdots & \cdots & \cdots\end{array}$

$4,560 \quad 5,460 \quad 1$

$\begin{array}{rrr}4,560 & \mathbf{5}, 460 & 4 \\ 4,560 & 5,460 & 3\end{array}$

$\begin{array}{lll}4,560 & 5,460 & 3 \\ 4,560^{18} & 5,460 & 5\end{array}$

$\begin{array}{lll}3,170 & 4,790 & 4 \\ 3,170 & 4,000 & 1\end{array}$

$\begin{array}{lll}3,170 & 4,000 & i\end{array}$

$\begin{array}{lll}3,840 & 4,790 & 2 \\ 3,840 & 4,790 & 2\end{array}$

$\begin{array}{lll}\ldots & \ldots & \cdots \\ \ldots & & \end{array}$

$\begin{array}{rrr}4,668 & \ldots & 1 \\ 2,640 & \ldots & 1\end{array}$

$\begin{array}{ccc}2,640 & \cdots & 1 \\ \cdots \cdots & \cdots & \cdots\end{array}$

$\begin{array}{lll}\ldots & \cdots & \cdots\end{array}$

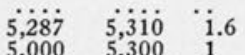

$\begin{array}{lll}5,000 & 5,300 & 1 \\ \ldots .^{5} & \cdots{ }^{5} & 1\end{array}$

$5,800 \quad \ldots . \quad 1.5$

$4,000 \quad \cdots \cdots \quad 1$

$\begin{array}{lll}\cdots & \cdots & \cdots\end{array}$

$\begin{array}{lll}\cdots & \cdots & \cdots \\ \ldots n^{5} & 4,300 & 1\end{array}$

$\ddot{2,700} \quad \ddot{3,600} \quad \dot{3}$

$\begin{array}{lll}\cdots & \cdots\end{array}$

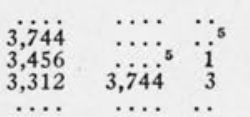

$\begin{array}{lll}\ldots & \ldots & \ldots \\ \cdots & \ldots & \cdots\end{array}$

$\begin{array}{lll}\because, 30{ }^{5} & 3,850 & i\end{array}$

4,300 $5,0000 \quad \ddot{3}$

$\begin{array}{ccc}4,200 & 5,300 & 3 \\ \ldots .^{5} & \ldots .{ }^{5} & 3\end{array}$

$\begin{array}{rrr}5,800 & 7,475 & 15 \\ 4,250 & 4,790 & 2\end{array}$

$\begin{array}{rrr}1,400 & 1,560 \\ 43 & 37 & 51\end{array}$
All Nonprofes.

SIONAL Assistants
Mini- Maxi- Num

mum mum ber

$\begin{array}{ccc}3,690^{5} & 3,200 & 1 \\ 3,120 & \cdots & 1 \\ \end{array}$

‥ $\cdots$.

$3,040_{5} \quad 3,380 \quad 3.5$

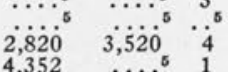

$3,672 \quad \ldots .^{5} 3$

$2,268 \quad 3,120 \quad 4$

$\begin{array}{lll}2,664 & \ldots & 1 \\ 1,320 & \ldots & .\end{array}$

$2,100 \quad \ldots{ }^{5} \quad 1$

$\cdots \cdots \quad \cdots$

$\cdots \cdots$

$2,400 \quad 2,880 \quad 1$

$\begin{array}{lll}2,160 & 3,120 & 2 \\ 3,060 & \ldots & 1\end{array}$

$\ddot{3,000} \quad 3,600 \quad$ i

$2,750 \quad 4,000 \quad 3$

$\begin{array}{lll}2,750 & 3,490 & 3 \\ 2,750 & 4,790 & 6\end{array}$

$\begin{array}{lll}2,620 & 3,640 \\ 2,620 & 2,908 & 1\end{array}$

$2,750 \quad 4,000 \quad 3$

2,764

1,866

2,824
3,128

4,000
$\ldots \ldots$

$\begin{array}{ll}2,887 & 3 \\ 3,388 & 1\end{array}$

$\begin{array}{lll}2,580 & \ldots & 1 \\ 2,640 & \cdots & 1\end{array}$

$\begin{array}{lll}2,640 & \cdots & 1 \\ 1,350 & \ldots & 1\end{array}$

․ $\cdots$.

$2, \dot{5} 2 \dot{0}^{5} \quad 3,1 \dot{80}^{5} \quad 3.5$

$2,100{ }_{5} \quad 2,376 \quad 2.6$

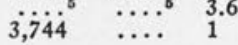

2,768 $\ldots \ldots \quad 1.5$

$\ddot{2,4000} \quad 3, \dddot{560} \quad \ddot{2}$

$\begin{array}{llll}3,027 & \ldots . & \ddots \mathrm{i}\end{array}$

$\begin{array}{lll}\ldots .5 & 2,320 & \text { i } \\ 1,000 & 2,300 \\ 1,950 & 2,400 & { }^{1} .5 \\ 2,400 & 3,607 & 3\end{array}$

$2, \ddot{5} \ddot{20} \quad \ddot{2}, \overline{7} \dot{6} \dot{7} \quad \ddot{2}$

$\begin{array}{lll}2,320 & 2,860 & 2 \\ 2,000 & 3,024 & 4 \\ 2,500 & 3\end{array}$

$\begin{array}{rcr}900 & \ldots & .5 \\ 2,100 & \cdots & .5 \\ 2,610 & \dddot{3}{ }^{5} & 2.5 \\ 2,652 & 3,432 & 4 \\ 2,520 & 3,060 & 1\end{array}$

$\begin{array}{lll}2,592 & \ldots & 1 \\ 3,372^{5} & 3,13 \dot{ }^{5} & 1 \\ \ldots{ }^{5} & \ldots{ }^{5} & 1\end{array}$

$\begin{array}{ccc}4,352 & 4,790 & 17.5 \\ 2,601 & 3,150 & 2\end{array}$

$\begin{array}{lll}900 & 2,000 & .5\end{array}$

$4474^{.5}$
TOTAL NuMBER OF EMPLOYeES IN FULL-TIME EQUIVALENTS

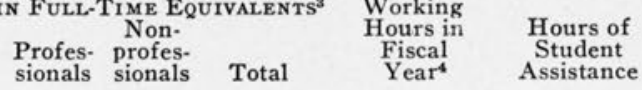

\begin{tabular}{|c|c|c|c|c|}
\hline $\begin{array}{l}3 \\
6 \\
1 \\
2 \\
1\end{array}$ & $\begin{array}{l}1.5 \\
1 \\
1 \\
\cdots \\
\cdots\end{array}$ & $\begin{array}{l}4.5 \\
7 \\
2 \\
2 \\
1\end{array}$ & $\begin{array}{l}1,908 \\
1,724 \\
1,400 \\
1,400 \\
1,120\end{array}$ & 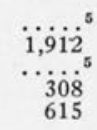 \\
\hline $\begin{array}{r}10 \\
7 \\
3 \\
9 \\
2\end{array}$ & $\begin{array}{l}3.5 \\
2.5 \\
1 \\
2 \\
1\end{array}$ & $\begin{array}{c}13.5 \\
9.5 \\
4 \\
11 \\
3\end{array}$ & $\begin{array}{c}1,373 \\
1,600 \\
1,368 \\
1,290 \\
\ldots{ }^{5}\end{array}$ & $\begin{array}{r}18,742 \\
13,160 \\
2,850 \\
12,916 \\
2,506\end{array}$ \\
\hline $\begin{array}{l}4 \\
3 \\
5 \\
3 \\
2.5\end{array}$ & $\begin{array}{l}3.5 \\
\because 4 \\
1 \\
1\end{array}$ & $\begin{array}{l}7.5 \\
3 \\
9 \\
4 \\
3.5\end{array}$ & $\begin{array}{l}1,760 \\
1,880 \\
\dot{1}, 640 \\
1,540\end{array}$ & $\begin{array}{r}4,041 \\
4,466 \\
3,627 \\
3,948 \\
11,087\end{array}$ \\
\hline $\begin{array}{l}5 \\
2 \\
2 \\
3 \\
2\end{array}$ & $\begin{array}{l}1 \\
\cdots \\
\therefore \\
\therefore\end{array}$ & $\begin{array}{l}6 \\
2 \\
2 \\
3 \\
2\end{array}$ & $\begin{array}{r}1,748 \\
1,760 \\
880 \\
2,024 \\
1,400\end{array}$ & $\begin{array}{l}1,248 \\
2,880 \\
6,621 \\
\dddot{2}, 74 \dot{6}^{5}\end{array}$ \\
\hline
\end{tabular}

$1,600 \quad 3,359$

$1,250 \quad 7,398$

$\begin{array}{ll}1,425 & 3,398 \\ 1,388 & 3,000\end{array}$

3,000
4,787

1,710

1,840

$1 \ddot{1,56 \dot{2}}$

1,722

2,175
1,750

2,863

2,918

$1,760 \quad 2,535$

$1,762 \quad 1,745$

1,920

1,400

17,419

$\ldots \ldots$

1,794

660
1,760

2,220

15,040

720

650

$\begin{array}{llll}2 & .5 & 2.5 & 2,000 \\ 2.6 & 3.5 & 6.16 & 1,832 \\ 3 & 1.5 & 4.5 & 1,851\end{array}$

$\begin{array}{cccc}\dddot{i}^{5} & .^{5} & .^{5} & 1,920 \\ & 1 & & 1,615\end{array}$

5,195

3,912
$6,458^{17}$

$6,458^{17}$
5,445

${ }_{3,747}^{5,445}$

$\begin{array}{lll}3.3 & 1.5 & 4.8 \\ 2 & i & 2 \\ 4 & 1 & 5 \\ 3.7 & 1.9 & 4.6 \\ 3 & 1 & 4\end{array}$

$1, \ddot{6} \dot{8} \dot{0}$

1,160
1,320

3,703

3,969

5,325

3,417
1,604

1,440

$\begin{array}{lll}5 & 1 & 5 \\ 1 & 1 & 2 \\ 6 & 1.5 & 7.5 \\ 7 & 1 & 8\end{array}$

1,300

1,900
1,920

1,743

$\ddot{3}, \dot{9} \dot{8} \dot{1}$

3,981
9,644

99,991
7,177

$\begin{array}{rrrrr}7 & & 7 & 1,930 & 3,018 \\ 4 & i & 5 & \cdots{ }_{5} & 4,953\end{array}$

$1,880^{\circ} \quad 11,186$

11,186
3,810

2,000

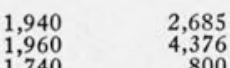

$\begin{array}{ll}1,760 & 7,666 \\ 1,760 & 4,224\end{array}$

1,575
1,368

1,368

4,314

2,340

3,602

9,200
1,743
660

49,86

9,867
4,224
308
85

Library science instructors, 11. For ten months, 12. For $101 / 2$ months. 13. Sabbatical leave, 14. For nine months. 15. Includes two clude summer session salaries. 19. Number of libraries on which high median, and low are based. 
Student

Library

1. Alabama, Christian C. ${ }^{1}$

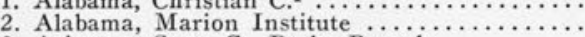

3. Arkansas State C., Beebe Branch ...............

4. California, American River $\ldots \ldots \ldots \ldots \ldots \ldots \ldots \ldots$

6. California, Coalinga C.

7. California, College of Marin $\ldots \ldots \ldots \ldots \ldots$

8. California, College of the Sequoias

9. California, Compton C.

10. California, East Contra Costa $\ldots \ldots \ldots \ldots \ldots \ldots \ldots$

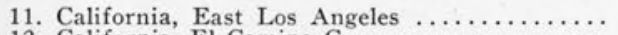

12. California, El Camino C. .............

13. California, Fresno $\ldots \ldots \ldots \ldots \ldots \ldots \ldots \ldots \ldots$

15. California, Glentan

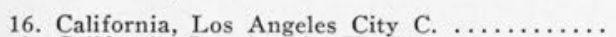

17. California, Los Angeles Harbo

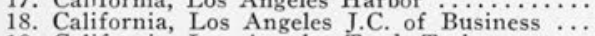

19. California, Los Angeles Trade-Tech ............

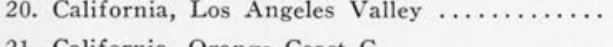

21. California, Orange Coast

23. California, Pasadena City $\mathrm{C} . \ldots \ldots \ldots \ldots \ldots \ldots \ldots \ldots$

24. California, Pierce

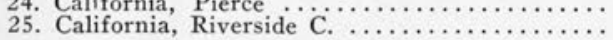

26. California, Sacramento ........................

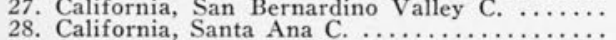

29. California, Santa Monica City C.

30. California, Stockton C. ${ }^{1} \ldots \ldots \ldots \ldots \ldots \ldots \ldots$

31. California, Yuba C

32. Colorado, Pueblo C.

33. Colorado, Trinidad State

34. Colorado, Woman's C. . ..............

36. Connecticut, Quinnipiac $\mathrm{C}$.

37. District of Columbia, Mount Vernon ${ }^{1} \ldots \ldots \ldots$

38. District of Columbia, Washington Hall .......

38. District of Columbia, Washington Hall $\ldots \ldots \ldots$

40. Florida, Edward Waters $\mathrm{C}$. . . . . . . . . . . .

41. Florida Christian C.

42. Florida, Jacksonville .

44. Georgia, Abraham Baldwin Agricultural $\mathrm{C}$.

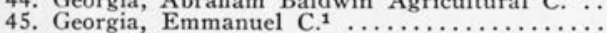

46. Georgia Southwestern C. $\ldots \ldots \ldots \ldots \ldots \ldots$

48. Georgia, Young L. G. Harris $\mathrm{C} \mathrm{i}^{\ldots} \ldots \ldots \ldots \ldots$

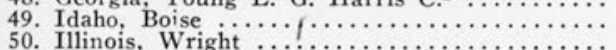

51. Illinois, Kendall C.

52. Illinois, La Salle-Peru-Öglesbvi $\ldots \ldots \ldots \ldots \ldots$

53. Illinois, Moline Community C

54. Illinois, Monticello C.

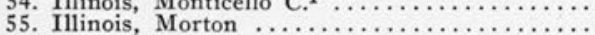

56. Illinois, Thornton

57. Iowa, Boone

58. Iowa, Mason City

59. Iowa, Mount Mercy C.

60. Iowa, Northwestern $\mathbf{1}$

61. Kansas, Coffeyville C.

62. Kansas, Hutchinson

63. Kansas, Kansas City

64. Kansas, St. Johns C. ${ }^{1} \ldots \ldots \ldots \ldots \ldots \ldots \ldots$

65. Kansas, Ursuline College of Paola ${ }^{2} \ldots \ldots \ldots$

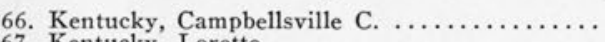

67. Kentucky, Loretto

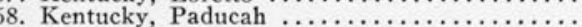

69. Maine, Oblate College and S. S. . . . . .

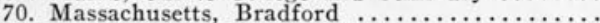

71. Massachusetts, Worcester $\ldots \ldots \ldots \ldots \ldots$

73. Michigan, Henry Ford Community C. $\ldots \ldots \ldots$

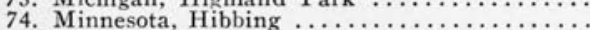

75. Minnesota, Itasca

76. Minnesota, Rochester

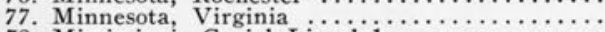

78. Mississippi, Copiah-Lincoln ${ }^{i} \ldots \ldots \ldots \ldots \ldots \ldots \ldots$
ENROLLMENT

Day $\begin{aligned} & \text { Eve- } \\ & \text { ning }\end{aligned}$

$\begin{array}{rr}63^{5} & 103 \\ 370^{4} & \end{array}$

$125^{5}$

728

$205^{5}$

1,130
1,471

1,471
3,396

1,264

$6,609^{3}$

1,338
1,857

1,857
2,200

7,456

1,306
645
5,843

5,843
4,820

1,638
346

$6,263^{3}$

$6,263^{3}$

1,282

2,380

1,764
821

821
3,559

1,432

\section{0}

710
334
$417^{4}$
227

353

353
$169^{4}$

16

422
365

$242^{4}$

785

$468^{4}$
$129^{4}$

$478^{4}$

416

$1,024^{5}$

$1,024^{5}$
3,087$$
2,000
$$

$176^{3,5}$

2, 000

286
553

464

114
328
$245^{4}$

$181^{5}$

400

$$
\begin{aligned}
& 400 \\
& 527 \\
& 510 \\
& 420
\end{aligned}
$$

510
$420^{2,4}$
$63^{2,3}$

40
1,682

1,682
1,812

$6,23 \dot{2}$

1,832

4,627

2,068

5,600

1,5i0

5,500
$\ldots$

10,047

2,965
1,245

12,617

12,617
6,777

6,204

1,228 14,464

$3,762 \quad 22,475$

433

2,543

2,888

35,000
23,325

23,325

18,00

$1,600 \quad 10,000$

$1,026 \quad 9,509$

$\because 12,929$

$120 \quad 7,000^{2}$

$400 \quad 6,299$

$61 \quad 10,025$

. . 6,986

51713,000

$641 \quad 12,415$

… ${ }^{7}{ }^{7,889}$

14,224

i26 12,077

$\begin{array}{ll}32^{2} & 14,800\end{array}$

$\begin{array}{cc}322 & 14,800 \\ 827 & 17,390\end{array}$

$4,098 \quad 54,039$

... 5,382

$\because \cdots \quad 14,239$

1,000 9,942

$\begin{array}{rl}277 & 15,556\end{array}$

$126 \quad 11,463$

3,056

$\begin{array}{llll}5,181 & 1,384 & 37\end{array}$

$\begin{array}{llll}63 & 10,000 & 1,521 & 108\end{array}$

... 4,870

8,870

8,362
8,187
26,000

$360^{4}$

$$
\begin{array}{r}
8 \\
18
\end{array}
$$

181
40
304

555

1,000

1,409

175

303

303
247
345

Vol$\begin{array}{rr}250 & 5 \\ 374 & 45 \\ 5,853 & 12\end{array}$

$\begin{array}{ll}5,853 & 120 \\ 1,028 & 275\end{array}$

$1,150 \quad 215$

$40 \quad 10,769^{2} \quad 386 \quad 175$

$\begin{array}{llll}\ldots . & 9,418 & 325 & 80\end{array}$

-Library Operat

and

umes Peri- Staff Student Related

$850^{2} \quad 60^{2} \$ 1,629^{2} \$ 504^{2} \$ 1,600^{2}$

1,800

4,062

12,084

270

870
1,255

$0,071 \quad 6,450$

$\begin{array}{rrr}2,996 & 116 & 4,890 \\ 620 & 150 & 12,885\end{array}$

12,885
8,655

$700 \quad 6,260$

8,655
18,205

10,420

3,iij $\quad 5,052$

$1,131 \quad 5,409$

$12,000 \quad 900 \quad 15,204$

$37,310 \quad 3,080 \quad 11,496$

$8,470 \quad 488 \quad 5,300$

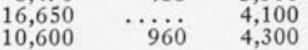

$76,144 \quad 15,240 \quad 36,310$

$22,331 \quad 1,524 \quad 9,307$

$9,580 \quad \ldots \ldots . \quad 4,623$

$\dddot{2}, 662$

8,679

$9,775 \quad 1,050 \quad 5,975$

6,420

$1,137 \quad 4,700$

$865 \quad 10,252$

$\begin{array}{rrr}10,000 & 763 & 7,760 \\ 14,242 & 1,450 & 4,520\end{array}$

19,515

2,600

19,500

12,990

27,607

1,431

$1,630 \quad 8,146$

$69^{2} \quad 7,752^{2}$

10,070

$1,330 \quad 5,200$

$\begin{array}{lll}4,870 & 774 & 2,245 \\ 4,200 & 242 & 1,092\end{array}$

3,718

$4,080 \quad 571 \quad 1,496$

8,915

600

1,782

6,700

600

4,392

6,994

750

1,008

$2,165 \quad 2,097$

3,137

6,450

5,300

6,600

750

8,135

2,248

905

2,917
1,674

$4,500 \quad 395-1,013$

$4,000 \quad 799 \quad 1,161$

$2,625^{12} \quad 1,710^{2} \quad 6,983^{2}$

$\begin{array}{rrr}8,000 & 2,155 & 5,491 \\ 72,930 & 12,540 & 16,250\end{array}$

3,300

$11,200^{2}$

$5,490^{2}$

3,590
14,930

$640 \quad 738$

$718^{2} \quad 1,056^{2}$

$360 \quad 926$

$6,300 \quad 400 \quad 2,133$

$5,000^{2} \quad 100^{2} \quad 320$

$\begin{array}{lll}5,200 & 900 & 2,000 \\ 3,600^{2} & 300 & 4,838 \\ & 650^{2} & 1,600^{2}\end{array}$

$\ddot{4,7} \ddot{5} 0$

4,750

4,500

$4,500^{15}$

695

1,000

$15 \quad 562$

$\ddot{2,640} \quad \cdots 7 \ddot{70}$

$\ddot{8}, 9 \ddot{0}^{15} \quad \ddot{1}, 6 \dot{2} \dot{7}$

400

3,500 


\begin{tabular}{|c|c|c|c|c|c|c|c|c|c|c|c|c|c|}
\hline $\begin{array}{l}\text { NG ExPI } \\
\text { Bind- } p \\
\text { ing }\end{array}$ & $\begin{array}{l}\text { PENDITU } \\
\text { Other } \\
\text { Operat- T } \\
\text { ing Ex- } \\
\text { pendi- F } \\
\text { tures }\end{array}$ & $\begin{array}{l}\text { Total Op- } \\
\text { erating } \\
\text { Expendi- } \\
\text { tures }\end{array}$ & $\begin{array}{l}\text { Per Stu- } \\
\text { dent Op- } \\
\text { erating } \\
\text { Expendi- I } \\
\text { tures }\end{array}$ & \begin{tabular}{l}
\multicolumn{1}{c}{ Total } \\
Educational \\
and General \\
Institutional \\
Expenditures
\end{tabular} & $\begin{array}{l}\text { Ratio of } \\
\text { Library } \\
\text { Expend- } \\
\text { itures to } \\
\text { Total } \\
\text { (Per } \\
\text { Cent) }\end{array}$ & $\overbrace{\text { brarian }}^{\mathrm{Li}-\mathrm{S}} \stackrel{\mathrm{S}}{\mathrm{A}}$ & $\begin{array}{l}\text { LARIES AS } \\
\text { EMBER 1, } \\
\text { Profes- } \\
\text { sional f } \\
\text { Assist- } \\
\text { ants }\end{array}$ & $\begin{array}{l}\text { S OF } \\
\text { Nontro- } \\
\text { fessional } \\
\begin{array}{c}\text { Assist- } \\
\text { ants }\end{array}\end{array}$ & $\begin{array}{l}\text { TOTAL NU } \\
\text { OF EMPLO } \\
\text { IN FULL-T } \\
\text { E } Q \text { UUAL } \\
\text { Profes- No } \\
\text { sional fes: }\end{array}$ & $\begin{array}{l}\text { IBER } \\
\text { EES } \\
\text { IME } \\
\text { NT } \\
\text { pro- } \\
\text { onal }\end{array}$ & $\begin{array}{c}\text { Average } \\
\text { Working } \\
\text { Hours } \\
\text { for Pro- } \\
\text { fessional } \\
\text { Librari- } \\
\text { ans }^{17}\end{array}$ & $\begin{array}{l}\text { Hours } \\
\text { of Stu- } \\
\text { dent } \\
\text { Assist- } \\
\text { ance }\end{array}$ & \\
\hline $\begin{array}{r}21 \\
65 \\
50 \\
300\end{array}$ & $\begin{array}{c}159 \$ \\
149 \\
100 \\
700\end{array}$ & $\begin{array}{r}3,892 \\
5,400 \\
3,490 \\
7,837 \\
29,605\end{array}$ & $\begin{array}{r}\$ 23.45 \\
14.59 \\
21.81 \\
4.68 \\
8.02\end{array}$ & $\begin{array}{r}72,461 \\
157,821 \\
59,242 \\
389,909 \\
1,080,501\end{array}$ & $\begin{array}{l}5 . \\
3.42 \\
5.89 \\
2 . \\
2.7\end{array}$ & $\begin{array}{c}\$ 1,125^{6} \$ \\
2,500^{6} \\
2,000^{6} \\
5,200^{7} \\
6,624^{7}\end{array}$ & $\begin{array}{l}\ldots \ldots \\
\ldots \ldots \\
\ldots \\
5,460^{7}\end{array}$ & $\begin{array}{c}\$ \quad 504^{6} \\
855^{6} \\
\because 900^{10} \\
1,8000^{6} \\
2,600\end{array}$ & $\begin{array}{l}1 \\
1 \\
1 \\
1 \\
1 \\
2\end{array}$ & $\begin{array}{l}i-1 / 3 \\
i-1 / 10\end{array}$ & $\begin{array}{r}333 \\
1,404 \\
315 \\
1,260 \\
1,330\end{array}$ & $\begin{array}{r}200 \\
\cdot 340 \\
540 \\
360 \\
5,000\end{array}$ & \\
\hline $\begin{array}{l}\dddot{168} \\
\dddot{304} \\
215\end{array}$ & $\begin{array}{r}200 \\
135 \\
1,045 \\
2,076 \\
2,150\end{array}$ & $\begin{array}{l}12,050 \\
16,867 \\
14,752 \\
32,082 \\
19,325\end{array}$ & $\begin{array}{r}58.78 \\
2.27 \\
4.47 \\
4.00 \\
5.80\end{array}$ & $\begin{array}{r}225,630 \\
529,295 \\
1 \dddot{1,346,764} \\
850,440\end{array}$ & $\begin{array}{l}5.3 \\
3 . \\
\ddot{2.38} \\
2.27\end{array}$ & $\begin{array}{l}5,310^{8} \\
6,682^{7} \\
6,260^{8} \\
7,350^{8} \\
6,940^{8}\end{array}$ & $\begin{array}{c}3,300^{8} \\
6,202^{7} \\
2,800^{9} \\
4,610^{8} \\
\ldots\end{array}$ & $\begin{array}{c}1,225^{8} \\
\ldots \\
3 \ddot{2} \dot{4} 0^{10} \\
3,480^{10}\end{array}$ & $\begin{array}{l}1 \\
2 \\
1 \\
2 \\
1\end{array}$ & $\begin{array}{l}\cdots \\
i \\
2 \\
1\end{array}$ & $\begin{array}{l}1,400 \\
1,267 \\
1,225 \\
1,430 \\
1,650\end{array}$ & $\begin{array}{r}700 \\
\cdots \cdots \\
\cdots \\
1,330\end{array}$ & 1 \\
\hline $\begin{array}{l}160 \\
700 \\
400 \\
200 \\
400\end{array}$ & $\begin{array}{r}1,678 \\
2,503 \\
500 \\
340 \\
\cdots \cdots\end{array}$ & $\begin{array}{l}29,942 \\
55,089 \\
15,158 \\
21,290 \\
16,260\end{array}$ & $\begin{array}{r}3.50 \\
8.25 \\
5.35 \\
11.46 \\
8.29\end{array}$ & $\begin{array}{l}\mathrm{i}, 730,60 \mathrm{i} \\
\mathrm{i}, 129,73 \mathrm{3} \\
1,113,353\end{array}$ & $\begin{array}{l}3 . \\
2.035 \\
1.80 \\
1.60\end{array}$ & $\begin{array}{l}4,250^{8,11} \\
6,220^{8} \\
5,870^{8} \\
6,800^{8} \\
4,970^{8}\end{array}$ & $\begin{array}{l}\dot{5,630} 0^{8} \\
\dot{5} \dot{0} 00^{8} \\
\ldots\end{array}$ & $\begin{array}{l}3,108^{10,11} \\
3,256^{10} \\
2,600^{8} \\
3,230^{8} \\
2,600^{10}\end{array}$ & $\begin{array}{ll}2 & 2 \\
3-1 / 2 & 5 \\
1 & 1 \\
2 & 2 \\
1 & 2\end{array}$ & $\begin{array}{l}2 \\
5 \\
1 \\
2 \\
2\end{array}$ & $\begin{array}{l}1,760 \\
1,232 \\
1,350 \\
1,246\end{array}$ & $\begin{array}{r}1,440 \\
3,080 \\
488 \\
7000\end{array}$ & \\
\hline $\begin{array}{r}2,496 \\
368 \\
200 \\
211\end{array}$ & $\begin{array}{r}5,195 \\
855 \\
3325 \\
\cdots\end{array}$ & $\begin{array}{r}135,385 \\
34,385 \\
14,403 \\
26,616 \\
\cdots \cdots\end{array}$ & $\begin{array}{l}7.70 \\
8.05 \\
7.62 \\
1.44 \\
\cdots\end{array}$ & 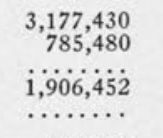 & $\begin{array}{l}4.26 \\
4.3 \\
i .40 \\
\ldots\end{array}$ & $\begin{array}{l}4,850^{8,11} \\
8,422^{8,13} \\
6,250^{8} \\
6,250^{8} \\
4,250^{8,11}\end{array}$ & $\begin{array}{l}4,250^{8,11} \\
7,220^{8,13} \\
5,500^{8} \\
4,250^{8,11}\end{array}$ & $\begin{array}{l}12,652^{10,11} \\
33,190^{10} \\
2,880^{8} \\
130 \dot{8}^{10,11}\end{array}$ & $\begin{array}{ll}8 & 6 \\
2-2 / 5 & 1 \\
1-1 / 5 & 1 \\
2 & \\
2 & 2\end{array}$ & $\begin{array}{l}6 \\
1 \\
1 \\
2\end{array}$ & $\begin{array}{l}\ddot{1,110} \\
1,200 \\
1,098 \\
\ldots \ldots\end{array}$ & $\begin{array}{l}2,000 \\
1,260 \\
\cdots \cdots \\
2,200\end{array}$ & \\
\hline $\begin{array}{r}387 \\
15 \\
1,500 \\
350\end{array}$ & $\begin{array}{r}2,000 \\
\ddot{2}, \overrightarrow{7} \dot{9} \dot{3} \\
300 \\
550\end{array}$ & $\begin{array}{l}19,187 \\
12,272 \\
50,408 \\
18,823 \\
21,112\end{array}$ & $\begin{array}{r}2.45 \\
7.79 \\
8.04 \\
47.05 \\
4.19\end{array}$ & $\begin{array}{r}745,077 \\
403,525 \\
2,868,247 \\
609,573 \\
753,907\end{array}$ & $\begin{array}{l}2.45 \\
3 . \\
1.75 \\
3 . \\
2.80\end{array}$ & $\begin{array}{l}5,165^{9} \\
6,420^{8} \\
8,108^{9} \\
6,400^{6} \\
6,558^{9}\end{array}$ & $\begin{array}{l}\cdots \\
6,835^{\circ} \\
4,500^{11} \\
6,660\end{array}$ & $\begin{array}{l}3,156^{10} \\
20 \dot{4}^{8} \\
3,456^{10} \\
3,660^{9}\end{array}$ & $\begin{array}{l}1 \\
1 \\
4 \\
1 \\
1 \\
2\end{array}$ & $\begin{array}{l}{ }^{2} 1 / 4 \\
2-1 / 4 \\
1 \\
1\end{array}$ & $\begin{array}{l}1,760 \\
1,600 \\
1,540 \\
1,062 \\
1,341\end{array}$ & $\begin{array}{r}1,050 \\
600 \\
961 \\
690 \\
1,450\end{array}$ & \\
\hline $\begin{array}{c}1,800 \\
832 \\
400 \\
1,454 \\
1,331^{2}\end{array}$ & $\begin{array}{r}850 \\
167 \\
1,728 \\
553^{2}\end{array}$ & $\begin{array}{l}33,015 \\
27,663 \\
18,496 \\
40,565 \\
36,491^{2}\end{array}$ & $\begin{array}{r}11.66 \\
6.42 \\
14.00 \\
6.09 \\
9.35^{2}\end{array}$ & $\begin{array}{c}1,297,381 \\
1,204,527 \\
607,437 \\
1,745,879 \\
1,892,800^{2}\end{array}$ & $\begin{array}{l}2.40 \\
2.12 \\
3.044 \\
2.38 \\
1.90^{2}\end{array}$ & $\begin{array}{l}7,450^{7} \\
6,725^{8} \\
8,243^{9,13} \\
6,998^{8}\end{array}$ & $\begin{array}{l}4,440^{11} \\
7,375 \\
6,99 \dot{8}^{9,13} \\
6,148^{8}\end{array}$ & $\begin{array}{l}3,360^{9} \\
3,511 \\
3,150^{9,13} \\
3,216^{9}\end{array}$ & $\begin{array}{ll}3-1 / 3 & \\
2 & 2 \\
2 & \\
3 & 2 \\
3-3 / 4 & 1\end{array}$ & $\begin{array}{l}\dot{2} \\
\dot{2} \\
1\end{array}$ & $\begin{array}{l}1,200 \quad 2 \\
1,281 \\
1,225 \\
1,440^{13} \\
1,400\end{array}$ & $\begin{array}{c}26,110 \\
1,332 \\
360 \\
1,630^{13} \\
100\end{array}$ & \\
\hline $\begin{array}{l}400 \\
240 \\
\because i 3 \\
\cdots\end{array}$ & $\begin{array}{r}1,530 \\
\cdots 85 \\
385 \\
\cdots\end{array}$ & $\begin{array}{r}18,530 \\
8,130 \\
5,619 \\
13,804 \\
6,147\end{array}$ & $\begin{array}{r}8.06 \\
4.68 \\
4.00 \\
33.10 \\
12.47\end{array}$ & $\begin{array}{l}406 \\
237 \\
379 \\
187\end{array}$ & $\begin{array}{l}3 . \\
2 . \\
2.4 \\
3.63 \\
3.20\end{array}$ & $\begin{array}{l}5,188^{6} \\
4,870^{6} \\
4,200^{6} \\
4,200^{8} \\
4,080^{8}\end{array}$ & $\begin{array}{l}4,680^{6} \\
\ldots \\
3,600^{8} \\
\ldots\end{array}$ & $\begin{array}{l}2,844^{10} \\
\ldots \\
\ldots \\
\ldots \\
\ldots\end{array}$ & $\begin{array}{l}1 \\
1 \\
1 \\
2-1 / 2 \\
1\end{array}$ & $\begin{array}{l}2 \\
\cdots \\
\cdots \\
\cdots\end{array}$ & $\begin{array}{l}1,400 \\
1,440 \\
1,440 \\
1,950 \\
1,270\end{array}$ & $\begin{array}{l}1,200 \\
1,190 \\
7939 \\
718\end{array}$ & \\
\hline $\begin{array}{r}22 \\
22 \\
20 \\
633\end{array}$ & $\begin{array}{r}150 \\
200 \\
258 \\
79\end{array}$ & $\begin{array}{r}11,297 \\
7,879 \\
3,675 \\
8,931 \\
16,396\end{array}$ & $\begin{array}{r}21.35 \\
5.10 \\
24.50 \\
10.86 \\
36.14\end{array}$ & $\begin{array}{r}246,3 \\
528,6 \\
10,0 \\
109,0 \\
\cdots \cdots\end{array}$ & $\begin{array}{r}4.58 \\
1.50 \\
37 . \\
8.10 \\
\cdots\end{array}$ & $\begin{array}{l}4,500^{9} \\
3,700^{8} \\
600^{10,10} \\
4,392^{10} \\
3,600^{9}\end{array}$ & $\begin{array}{l}3 \ddot{3} 000^{8} \\
\cdots \cdots \\
2,900\end{array}$ & $\begin{array}{l}3,400^{9} \\
300^{10,16} \\
1,956^{10} \\
1,350^{6}\end{array}$ & $\begin{array}{l}1 \\
2 \\
1 \\
1 \\
2\end{array}$ & $\begin{array}{l}1 \\
i \\
1 \\
1\end{array}$ & $\begin{array}{r}1,702 \\
1,482 \\
1,000 \\
2,555 \\
810\end{array}$ & $\begin{array}{l}\ldots \\
1,800 \\
\ldots \ldots\end{array}$ & \\
\hline $\begin{array}{r}68 \\
350 \\
106 \\
929^{2}\end{array}$ & $\begin{array}{r}57 \\
253 \\
247 \\
569^{2}\end{array}$ & $\begin{array}{r}4,918 \\
15,216 \\
8,470 \\
9,204 \\
6,973^{2}\end{array}$ & $\begin{array}{l}20.32 \\
13.81 \\
10.79 \\
19.67 \\
54.05\end{array}$ & $\begin{array}{r}131 \\
350 \\
208 \\
208 \\
104\end{array}$ & $\begin{array}{l}3.70 \\
4.34 \\
\dot{4} .4 \\
6.68\end{array}$ & $\begin{array}{l}37 \\
50^{8} \\
00 \\
00^{6}\end{array}$ & $\begin{array}{l}\ldots \\
\ldots \\
\cdots \\
\cdots \\
\cdots\end{array}$ & $\begin{array}{l}\ldots \\
1,550^{10} \\
1,800^{8,11} \\
\ldots\end{array}$ & $\begin{array}{l}1 \\
1 \\
1 \\
1 \\
1 \\
1\end{array}$ & $\begin{array}{l}i^{1 / 3} \\
\cdots\end{array}$ & $\begin{array}{r}1,600 \\
1,892 \\
1,372 \\
2,112 \\
\cdots \cdots\end{array}$ & $\begin{array}{l}1,870 \\
2,580 \\
800^{14} \\
1,283 \\
2,178\end{array}$ & \\
\hline $\begin{array}{c}178 \\
126 \\
78^{2} \\
312 \\
650\end{array}$ & $\begin{array}{r}\ddot{287} \\
\ddot{1}, 9 \dot{1} \dot{3} \\
6,350\end{array}$ & $\begin{array}{r}6,086 \\
6,372 \\
11,396^{2} \\
17,871 \\
108,720\end{array}$ & $\begin{array}{c}12.73 \\
14.41 \\
26.09^{2} \\
9.65 \\
15.12\end{array}$ & $\begin{array}{r}141 \\
138 \\
172 \\
520 \\
\cdots\end{array}$ & $\begin{array}{l}4.6 \\
6.01 \\
3.43 \\
\cdots\end{array}$ & $\begin{array}{l}4,750^{10} \\
4,000^{8} \\
2,400^{10} \\
4,500^{6} \\
7,250^{8}\end{array}$ & $\begin{array}{c}\ldots \ldots \\
\cdots \\
4,000 \\
5,500^{8}\end{array}$ & $\begin{array}{l}\ldots \\
\cdots \\
\cdots \\
3 \dot{2} \dot{0} 0^{8}\end{array}$ & $\begin{array}{l}1 \\
1 \\
1 \\
2 \\
2 \\
7\end{array}$ & $\begin{array}{c}\cdots \\
1 / 4 \\
1 / 4\end{array}$ & $\begin{array}{l}2,400 \\
1,417 \\
2,080 \\
1,500 \\
1,200\end{array}$ & $\begin{array}{r}789 \\
5,560 \\
1,153 \\
\cdots\end{array}$ & \\
\hline $\begin{array}{c}2,9 \ddot{9} 75^{2} \\
100 \\
180 \\
\cdots\end{array}$ & $\begin{array}{l}\cdots \ldots \\
\cdots \\
305 \\
210\end{array}$ & $\begin{array}{c}4,678 \\
15,949^{2} \\
8,590^{2} \\
5,361 \\
20,667\end{array}$ & $\begin{array}{l}26.53 \\
56.96^{2} \\
20.86^{2} \\
18.70 \\
24.90\end{array}$ & $\begin{array}{c}95,500 \\
640,914^{2} \\
\cdots \ddot{2} \dot{3} \dot{3} 35^{2} \\
401,605\end{array}$ & $\begin{array}{l}4.91 \\
2.4 \\
1.9^{2} \\
5.01\end{array}$ & $\begin{array}{l}3,300^{8} \\
6,250^{7} \\
4,010^{2,7} \\
2,900^{8,12} \\
7,600^{8}\end{array}$ & $\begin{array}{l}6,125^{7} \\
720^{2,7} \\
6,625^{8}\end{array}$ & $\begin{array}{c}\cdots \\
\cdots \\
2,240^{8} \\
\cdots\end{array}$ & $\begin{array}{l}1 \\
2 \\
1 \\
1 \\
1 \\
8\end{array}$ & $\begin{array}{l}1 / 2 \\
1 / 2 \\
1 / 2 \\
\cdots\end{array}$ & $\begin{array}{l}1,704 \\
1,420 \\
1,440^{2} \\
1,050 \\
1,253\end{array}$ & $\begin{array}{r}640 \\
1,025 \\
-832 \\
800\end{array}$ & \\
\hline $\begin{array}{l}175 \\
100 \\
100 \\
754 \\
100^{2}\end{array}$ & $\begin{array}{c}375 \\
\cdots 65 \\
65 \\
61 \\
850^{2}\end{array}$ & $\begin{array}{l}9,383 \\
5,520^{2} \\
8,265 \\
5,953 \\
6,800^{2}\end{array}$ & $\begin{array}{l}20.22 \\
19.00 \\
25.19 \\
22.46 \\
29.57^{2}\end{array}$ & $\cdots$ & $\begin{array}{l}\cdots \\
\dot{5} .3 \\
4.6 \\
4.36^{2}\end{array}$ & $\begin{array}{l}6,300^{8} \\
5,000^{6} \\
5,200^{8} \\
\dot{3,600} 0^{8}\end{array}$ & $\begin{array}{l}4.500^{6} \\
\ldots \\
\ldots \\
\ldots\end{array}$ & $\begin{array}{l}\ldots \\
\ldots \\
\ldots \\
\ldots \\
\cdots\end{array}$ & $\begin{array}{l}1 \\
2 \\
1 \\
1 \\
1\end{array}$ & $\begin{array}{l}6^{1 / 3} \\
\cdots \\
\cdots\end{array}$ & $\begin{array}{r}1,440 \\
1,260 \\
1,067 \\
300 \\
1,629\end{array}$ & $\begin{array}{r}459 \\
\ddot{1,520} \\
178 \\
1,080\end{array}$ & \\
\hline $\begin{array}{r}100 \\
144 \\
200 \\
100 \\
75\end{array}$ & $\begin{array}{l}\ddot{580} 0 \\
\dddot{750} \\
\cdots\end{array}$ & $\begin{array}{l}1,100 \\
7,979 \\
5,200 \\
9,150 \\
5,564^{2}\end{array}$ & $\begin{array}{r}2.7 \\
15.1 \\
10.1 \\
21.7 \\
54.0\end{array}$ & $\begin{array}{l}\ddot{154,830} \\
\ddot{3} \ddot{38}, 000 \\
94,000^{2}\end{array}$ & $\begin{array}{l}\ddot{2.7} \\
5.09\end{array}$ & $\begin{array}{l}4,500^{8} \\
4,950^{6} \\
4,100^{6} \\
4,500^{6} \\
4,500^{9,15}\end{array}$ & $\begin{array}{l}\ldots \\
\ldots \\
\cdots \\
\ldots \\
\ldots\end{array}$ & $\cdots$ & $\begin{array}{l}7 \\
1 \\
1 \\
1 \\
1\end{array}$ & $\begin{array}{l}\cdots \\
\cdots \\
\cdots\end{array}$ & $\begin{array}{l}\dddot{1,400} \\
1,440 \\
1,440 \\
1,800\end{array}$ & $\begin{array}{l}1,158 \\
\cdots \ldots \\
\ddot{84} 0^{14}\end{array}$ & \\
\hline $\begin{array}{r}22 \\
16 \\
25 \\
78 \\
245\end{array}$ & $\begin{array}{r}45 \\
59 \\
1,180 \\
208 \\
389\end{array}$ & $\begin{array}{r}4,543 \\
641 \\
5,243 \\
1,448 \\
14,423\end{array}$ & $\begin{array}{r}12.61 \\
7.82 \\
21.40 \\
36.20 \\
47.44\end{array}$ & $\ddot{9} \dot{9}$ & $\begin{array}{l}7 . \\
\dot{6.4} \\
4 . \\
2.10\end{array}$ & $\begin{array}{l}2,800^{6} \\
2,640^{9} \\
\ldots \ldots \\
\ldots\end{array}$ & $\begin{array}{l}\ldots \\
\ldots \\
\ldots \\
\cdots \\
\cdots\end{array}$ & $\cdots$ & $\begin{array}{l}1 \\
1 \\
2\end{array}$ & $\begin{array}{l}1 / 8 \\
1 / 2 \\
1 / 2\end{array}$ & $\begin{array}{r}1,460 \\
1,860 \\
1,600 \\
1,500\end{array}$ & $\begin{array}{r}1,785 \\
\dddot{300} \\
\dddot{120}\end{array}$ & \\
\hline $\begin{array}{l}250 \\
200 \\
161 \\
308^{2}\end{array}$ & $\begin{array}{c}\cdots \cdots \\
8,850 \\
115 \\
192^{2}\end{array}$ & $\begin{array}{r}5,890 \\
16,238 \\
28,600 \\
7,441 \\
11,533^{2}\end{array}$ & $\begin{array}{r}3.47 \\
3.53 \\
19.50 \\
13.88 \\
46.12\end{array}$ & $\begin{array}{l}319,726 \\
878,294 \\
\ldots \ldots \ldots \\
\ldots \ldots \ldots\end{array}$ & $\begin{array}{l}1.85 \\
1.84 \\
\cdots \\
\cdots\end{array}$ & $\begin{array}{l}3,500^{10} \\
6,484^{8} \\
6,650^{8} \\
2,223^{8,16} \\
6,200^{7}\end{array}$ & $\begin{array}{l}5,634^{8} \\
\ldots \\
\ldots \\
\ldots\end{array}$ & $\begin{array}{l}3,400^{8} \\
3,324^{10} \\
1,418^{7,16}\end{array}$ & $\begin{array}{l}1 \\
2-1 / 6 \\
1 \\
1\end{array}$ & $\begin{array}{l}3^{16} \\
\dot{2-1}-2 \\
1 / 2\end{array}$ & $\begin{array}{c}1,880 \\
1,600 \\
\dddot{975} 5^{16} \\
1,260\end{array}$ & $\begin{array}{l}360 \\
720 \\
400 \\
380 \\
\cdots \cdots\end{array}$ & \\
\hline & $\begin{array}{l}143 \\
\ldots\end{array}$ & $\begin{array}{l}8,486 \\
6,521\end{array}$ & $\begin{array}{l}34.36 \\
17.43\end{array}$ & $\begin{array}{l}12 \\
21\end{array}$ & $\begin{array}{l}6.8 \\
2.8\end{array}$ & & $\begin{array}{l}\cdots \\
\cdots \\
\cdots\end{array}$ & $\begin{array}{l}520 \\
\cdots\end{array}$ & $\begin{array}{l}1 \\
1 \\
1\end{array}$ & $\cdots$ & $\begin{array}{l}1,760 \\
1,350 \\
1,890\end{array}$ & & \\
\hline
\end{tabular}


Junior College Library

Student

ENROLLMENT

Library

79. Mississippi, Perkinston

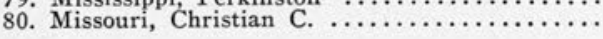

81. Missouri, Cottey C.

Missouri, Flat River

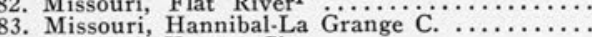

84. Missouri, Kansas City $\ldots \ldots \ldots \ldots \ldots$

86. Missouri, Moberly ${ }^{1}$

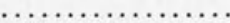

88. Missouri, Southwest Baptist C. ..............

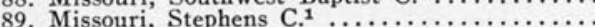

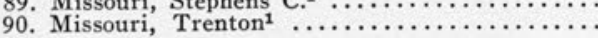

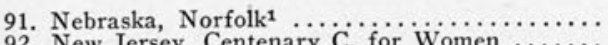

92. New Jersey, Centenary C. for Women .......

93. New Jersey, Trenton $\ldots \ldots \ldots \ldots \ldots \ldots \ldots \ldots$

95. New Mexico, Military Institute ${ }^{i} \ldots \ldots \ldots \ldots \ldots$

96. New York, Briarcliff

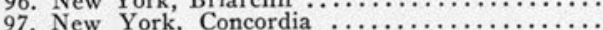

98. New York, Fashion Institute of Technology . .

99. New York, Hudson Valley Tech. Inst.

100. New York, Orange County Community ${ }_{\mathrm{C}}{ }^{\mathrm{i}} \ldots$

101. New York, Westchester Community C.

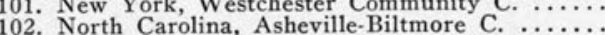

103. North Carolina, Brevard C.

104. North Carolina, Louisburg $\mathrm{C}_{1} \cdot \cdots$

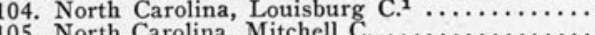

106. North Carolina, Warren Wilson C. ${ }^{1} \ldots \ldots \ldots$

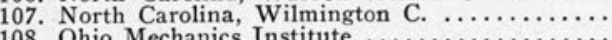

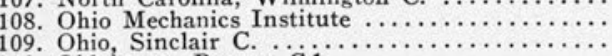

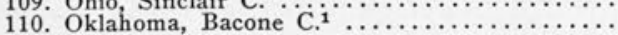

111. Oklahoma, Central Christian C.

112. Oklahoma, Connors State Agri C.

113. Oklahoma, Eastern Oklahoma A. \& M. C. . .

115. Oklahoma, Murray State Agric. C. $\because \dddot{\mathrm{M}} . \ddot{\mathrm{C}}$.

116. Oklahoma, Northern Oklahom

117. Oklahoma, St. Gregory's $\mathbf{C}^{1} \ldots \ldots \ldots \ldots$

118. Oregon, Oregon Technical Institute

119. Pennsylvania, Johnstown Center U. of Pittsburgh

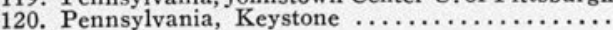

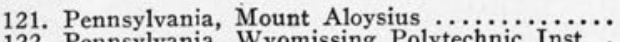

122. Pennsylvania, Wyomissing Polytechnic Inst. .

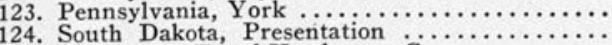

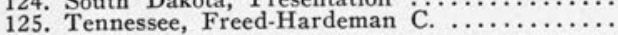

126. Texas, Arlington State C. $\ldots \ldots \ldots \ldots \ldots \ldots \ldots \ldots \ldots \ldots \ldots$

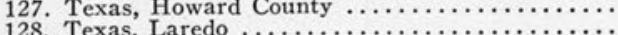

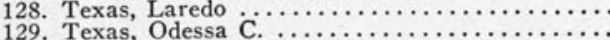

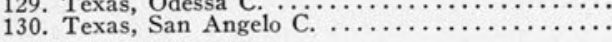

131. Texas, San Antonio C. $\ldots \ldots \ldots \ldots \ldots \ldots \ldots$

132. Texas, South Texas

133. Texas, Southwestern ${ }^{1}$

134. Texas, Tarleton State C. $_{\ldots \ldots \ldots \ldots \ldots \ldots \ldots \ldots \ldots}$

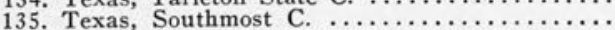

136. Texas, Victoria C. $\ldots \ldots \ldots \ldots \ldots \ldots \ldots \ldots$

138. Utah, Weber C.

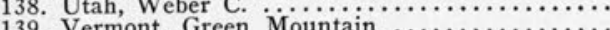

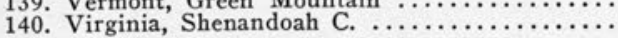

141. Virginia, Intermont C. ${ }^{1}$

142. Washington, Centralia

144. West Virginia, Potomac State $\mathrm{C} \ldots \ldots \ldots \ldots \ldots$

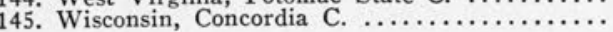

146. Wyoming, Casper $\ldots$ Wyoming, Northern Wyo. Community $\ldots \ldots$

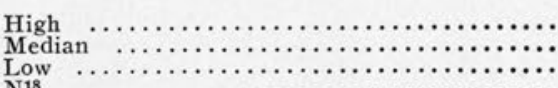

500
10
88
Day ning

$646^{2,4} \ldots$

8,539
17,794

$234^{4} \quad \ldots \ldots 13,479$

$\begin{array}{llll}185 & \ldots & 9,732\end{array}$

$\begin{array}{lll}318^{4} & \ddot{7} \ddot{8} & 11,104 \\ 238 & 32,000\end{array}$

$292^{2,4} \ldots \ldots .10,773^{2}$

$377^{2,4}, \ldots \cdots \quad 10,1$

$175^{2,4} \ldots$

$404^{4}$

439
86

240

471
291
252

$541^{2,4}$

$269^{4}$

137
450

590

571

540

1

249

$196^{\circ}$

17
31
34
15
18

150

$130^{4}$

$399^{4}$
$679^{5}$
$482^{4}$
$665^{5}$

454

454
36
1,250
528

528
$245^{5}$

$$
\begin{aligned}
& 10 \\
& 17 \\
& 27
\end{aligned}
$$

278

4074
$3,270^{5}$

\section{439
906}

439
$906^{5}$
$796^{5}$

7965

1,350
107
19

$$
\begin{aligned}
& 107 \\
& 192 \\
& 840^{5} \\
& 879
\end{aligned}
$$

840
879

\section{$487^{5}$
$476^{5}$}

2,488
356

143

\section{5}

304
948
537
38

$382^{4}$

418
174

7,45

$\ldots$

$\ldots$
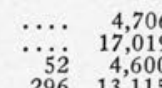

52
296

\section{…}

250
987

12,984
3,530

3,530
2,500

9,628

9,975

196

16,411

8,415

16,438

$\begin{array}{rr}\cdots 35 & 16,438\end{array}$

$2,019 \quad 19,357$

1,050

4,778
13,678

$26 \quad 4,011$

360

8,006

9,93

$102 \quad 5,76$

20

13,040

... 7,5

i73 7,07

7,076
12,664

$10 \quad 10,297^{2}$

$364 \quad 2,352$

$88 \quad 7,323$

… 3,400

525
195
265

195
265

3,157

308

ii

110
621

384
43

43
2,077

$1016,038^{2}$

$727 \quad 3,075$

$2,190 \quad 11,451$

.... 27,300

$900 \quad 7,000$

25610,500

12,617

87,910

10,780

600
145

145

1. College includes high school junior and senior, and college

1. College includes high school junior and senior, and college freshman and sophomore years. 2. Figure in and and evening students. 4. The majority of students are dormitory students. 5. Some students are dormitory stu-
dents. 6 . Nine months on duty. $7.91 / 2$ months on duty. 8 . Ten months on duty. 9. Eleven months on duty. 10 . 
Statistics, $1955^{-5} 6$ (Cont.)

ING EXPENDITURES Ratio of Thatar Number Werage Other Per Stu- Total Expend- DEPTEMBER 1, 1956- OF EMPLOYEes Hours Hours Operat-Total Op-dent Op- Educational itures to Profes- Nonpro- IN FULL-Time for Pro- of StuBind- $\begin{aligned} & \text { ing Ex- erating erating andi- Expendi- Expendi-Institutional (Per Li- Assist- Assist- Profes- Nonpro- Librari- Assist- } \\ & \text { pendit. }\end{aligned}$ ing tures tures tures Expenditures Cent) brarian ants ants sional fessional ans ance

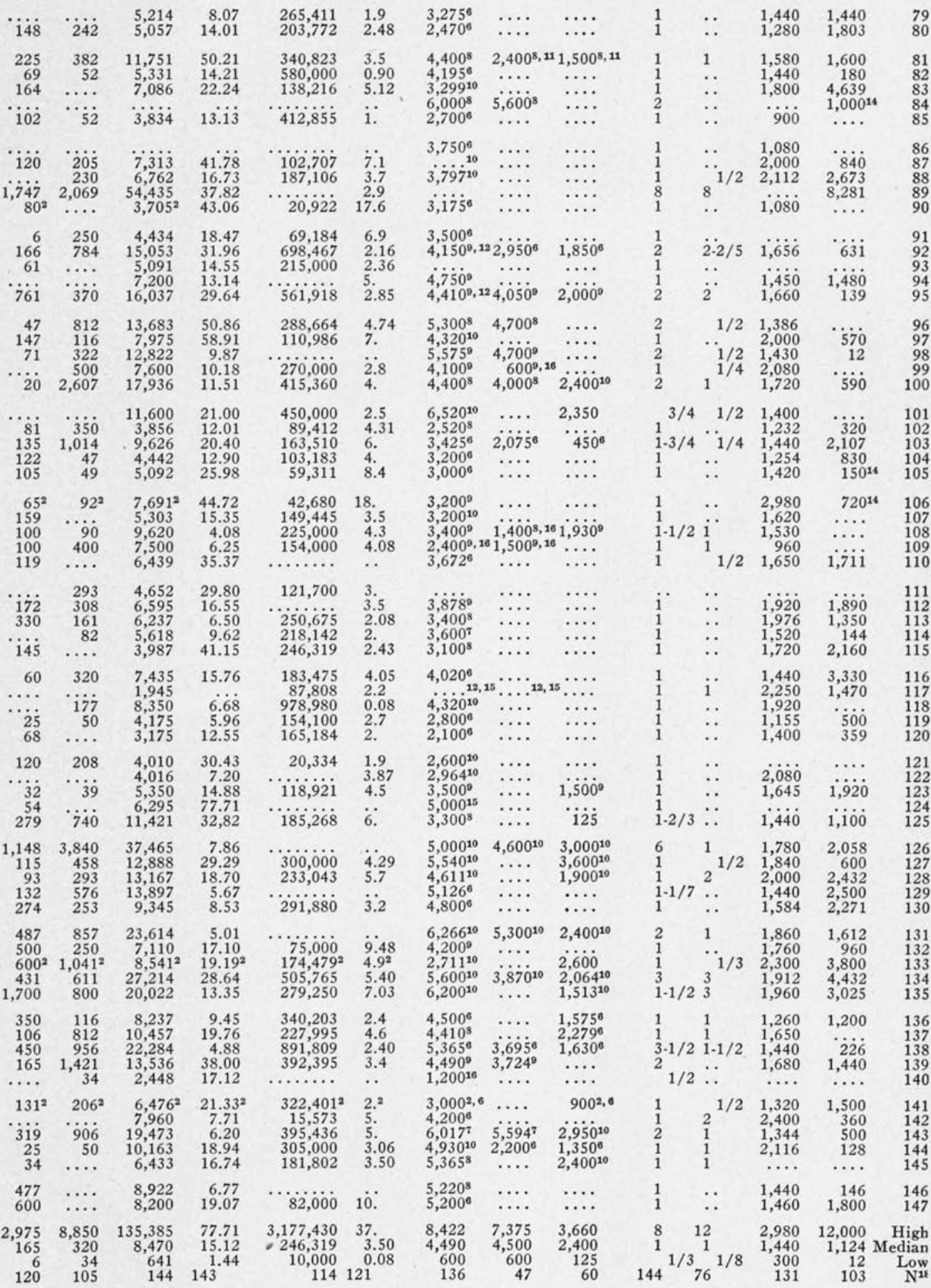

Twelve months on duty. 11. Minimum salary, actual salary not reported. 12. Receives room and board in addition to actual monetary salary. 13. Includes summer session. 14. Not paid out of library budget. 15. Contributed services; salary if given is estimated. 16. Part-time. 17. Excludes vacation and legal holidays. 18. Number of libraries on which high, median, and low are based. 